Campos de vetores em variedades singulares

\author{
Evandro Alves Nakajima
}



SERVIÇO DE PÓS-GRADUAÇÃO DO ICMC-USP

Data de Depósito:

Assinatura:

\title{
Campos de vetores em variedades singulares
}

\author{
Evandro Alves Nakajima
}

Orientadora: Profa. Dra. Maria Aparecida Soares Ruas

Dissertação apresentada ao Instituto de Ciências Matemáticas e de Computação - ICMC-USP, como parte dos requisitos para obtenção do título de Mestre em Matemática. VERSÃO REVISADA 
Ficha catalográfica elaborada pela Biblioteca Prof. Achille Bassi e Seção Técnica de Informática, ICMC/USP, com os dados fornecidos pelo(a) autor(a)

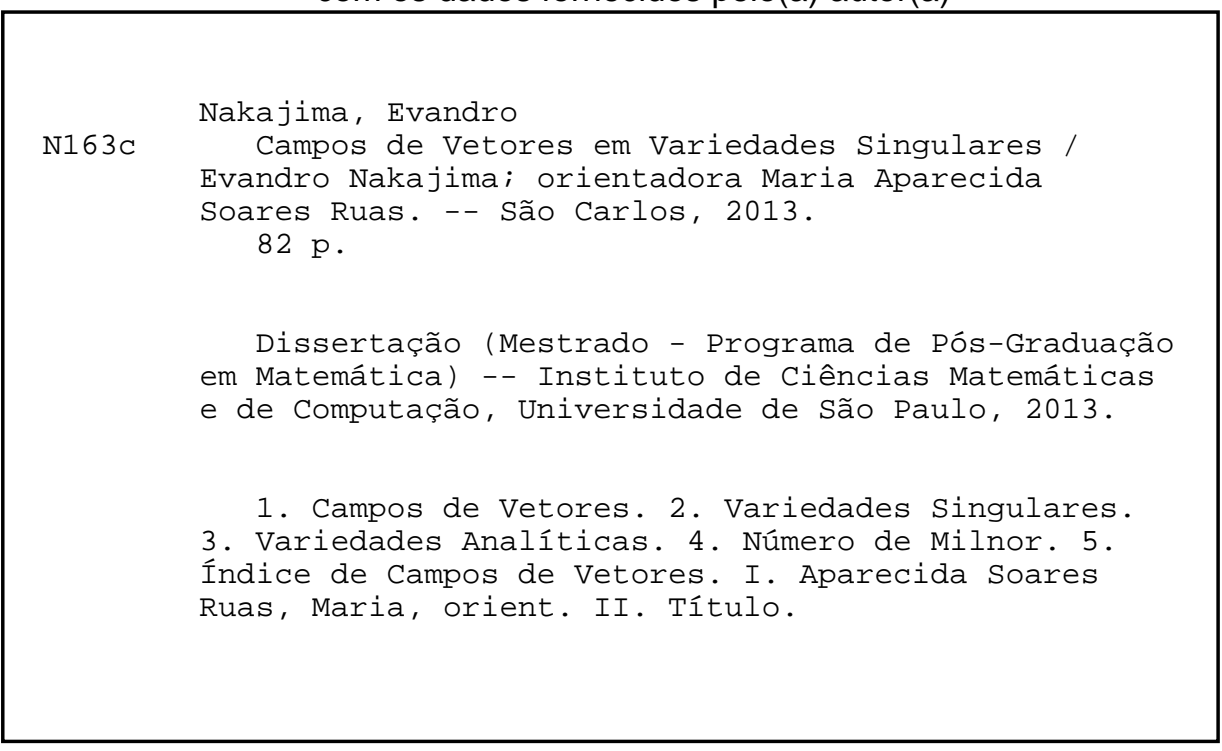




\section{Agradecimentos}

Agradeço primeiramente aos meus pais, Julio e Edinea, pois sem o esforço, carinho e incentivo não teria nem começado este trabalho.

A minha irmã Ticiana, pelas palavras e gestos de incentivo.

Aos meus amigos do ICMC: Ariadna, Gabriel, Laís, Gil, Preta e Banana.

Aos meus amigos Ferdnando, Ginnara, Giovane, Thiago e Patrícia, que mesmo de longe, me deram um suporte indispensável para continuar. Também aos meus amigos Liliane, André, Didi, Diego e Priscila.

Gostaria de agradecer imensamente minha orientadora Professora Dra. Maria Aparecida Soares Ruas, pelo conhecimento transmitido, pela paciência e por ser uma grande amiga, me apoiando nos momentos mais difíceis. 



\section{Resumo}

Neste trabalho estudamos índices de campos de vetores em variedades regulares e em variedades com singularidades isoladas. O principal resultado é o Teorema de Poincaré-Hopf que relaciona a característica de Euler de uma variedade com o índice de Poincaré-Hopf do campo. Para intersecções completas com singularidades isoladas, vemos também algumas variações deste teorema que relacionam a característica de Euler com o índice de Schwartz, o índice GSV e o número de Milnor da fibra genérica. 



\section{Abstract}

In this work we study some indices of vector fields on regular manifolds, and on manifolds with isolated singularity. The main result is the Poincaré-Hopf Theorem, which connects the Euler characteristic with the Poincaré-Hopf index of the field. For complete intersections with isolated singularities, we also study some variations of this theorem, which connects the Euler characteristic with the Schwartz index, the GVS index and the Milnor number of the generic fiber. 



\section{Sumário}

\begin{tabular}{lll}
\hline 1 & Preliminares & 1
\end{tabular}

1.1 Definições e Resultados Gerais . . . . . . . . . . . . . . . . . . . . . . . 1

1.2 Orientação de Variedades $\ldots \ldots \ldots$. . . . . . . . . . . . . . . . . 7

1.3 Homologia Simplicial . . . . . . . . . . . . . . . . . . . . . . . . . . . . . 8

1.4 Introdução à Teoria de Singularidades . . . . . . . . . . . . . . . . . . 13

2 Índices de Campos de Vetores: Caso Suave 19

2.1 Grau Módulo 2 de uma Aplicação $\ldots . . . .19$

2.2 Índices de Campos de Vetores e o Teorema de Poincaré-Hopf . . . . . . 27

\begin{tabular}{|lll}
\hline 3 & Variedades Singulares & 39
\end{tabular}

3.1 Conjuntos Algébricos $\ldots \ldots \ldots$

3.2 Conjuntos Analíticos . . . . . . . . . . . . . . . . . . . . . 52

4 Índices de Campos de Vetores: Caso Singular

4.1 Índice de Schwartz . . . . . . . . . . . . . . . . . 56

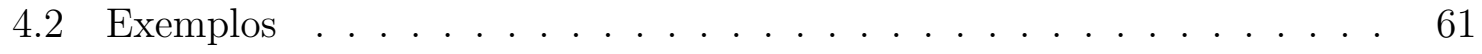

4.3 O Índice GSV para Campos de Vetores em ICIS . . . . . . . . . . . . . 65

5 Característica de Euler da fibra genérica de ICIS simples. 



\section{Introdução}

Há mais de um século, o estudo de campos vetoriais na vizinhança de um ponto singular isolado desempenha um papel importante em diversas áreas da matemática e em outras áreas da ciência, tais como física, biologia, meteorologia, entre outras. Um dos principais invariantes associados a um campo vetorial na vizinhança de um zero isolado é o índice de Poincaré-Hopf da singularidade, que tem sido estudado sob diferentes pontos de vista e sobre o qual existe uma vasta literatura. Ao mesmo tempo, a existência de espaços singulares como objetos centrais tanto na Matemática quanto em outras áreas propõe a questão natural de uma boa definição de índice de pontos singulares de campos vetoriais em variedades com singularidades. Nosso objetivo é obter, para variedades singulares, definições de índices de campos vetoriais, que reproduzam resultados locais e globais válidos no caso de variedades suaves.

Por exemplo, para variedades suaves, o Teorema de Poincaré-Hopf relaciona a soma dos índices de um campo vetorial com singularidades isoladas à característica de Euler da variedade, independentemente do campo vetorial escolhido. Encontrar novas noções de índices em variedades singulares que satisfaçam ao Teorema de Poincaré-Hopf é um problema importante no estudo de tais variedades.

O objetivo deste trabalho é estudar índices de campos de vetores em variedades, tanto suaves quanto com singularidades isoladas.

No primeiro capítulo veremos definições e resultados elementares, porém essenciais para o desenvolvimento do trabalho. As principais referências para este capítulo são [15], [14] e 6].

No segundo capítulo veremos o conceito de grau de uma aplicação e o utilizaremos para definir o índice de um campo de vetores em uma singularidade isolada. A partir 
daí demonstraremos uma primeira versão do teorema de Poincaré-Hopf. A principal referência para este capítulo é [19]

No terceiro capítulo estudaremos resultados sobre variedades singulares, principalmente utilizando conceitos e resultados algébricos. Os principais resultados deste capítulo são o Teorema 3.23, que dá uma caracterização para vizinhança de um ponto singular, e o Teorema 3.24 da Fibração de Milnor. A principal referência para este capítulo é [20].

No quarto capítulo estudaremos índices de campos de vetores agora em variedades singulares. Estudaremos também o teorema de Poincaré-Hopf para estes índices. A principal referência para este capítulo é [3].

No quinto capítulo faremos exemplos com os cálculos do número de Milnor da fibra genérica de variedades. As principais referências são [17] e [7]. 


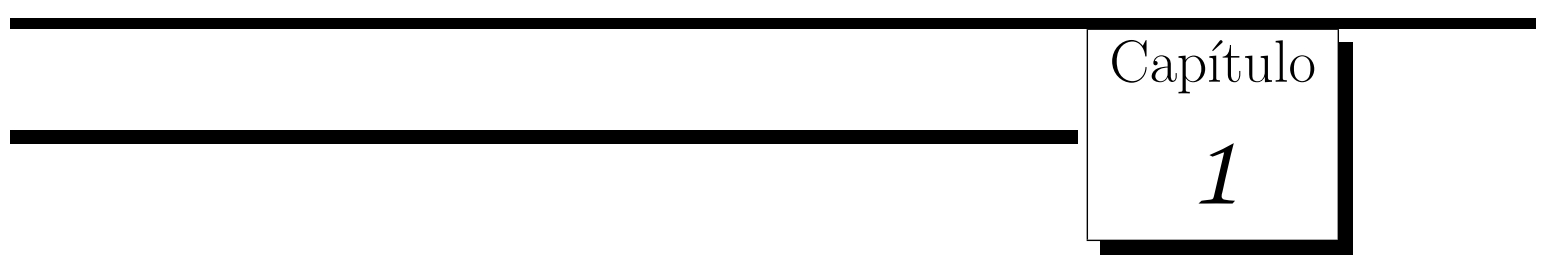

\section{Preliminares}

Neste capítulo veremos alguns conceitos e resultados básicos para o desenvolvimento desta dissertação. Veremos resultados sobre variedades suaves, homologia simplicial e faremos uma introdução à teoria de singularidades.

\subsection{Definições e Resultados Gerais}

Sejam $U \subset \mathbb{R}^{k}$ e $V \subset \mathbb{R}^{l}$ subconjuntos abertos. Uma aplicação $f: U \rightarrow V$ é suave se todas as derivadas parciais

$$
\frac{\partial^{n} f}{\partial x_{i_{1}} \ldots \partial x_{i_{n}}}, \quad i_{j} \in\{1, \ldots, k\}, \quad i_{1}+\ldots+i_{k}=n
$$

existem e são contínuas. Mais geralmente, dizemos que $f: X \rightarrow Y$, com $X \subset \mathbb{R}^{k}$ e $Y \subset \mathbb{R}^{l}$ conjuntos quaisquer, é suave se, e somente se, para cada $x \in X$ existe aberto $U \subset \mathbb{R}^{k}$ contendo $x$ e uma função $F: U \rightarrow \mathbb{R}^{l}$, tal que $\left.F\right|_{U \cap X}=f$. Dessa definição, segue que a aplicação identidade é suave e, da regra da cadeia, que a composição de aplicações suaves é suave.

Definição 1.1 : Uma aplicação $f: X \rightarrow Y$ é um difeomorfismo se é um homeomorfismo suave cuja inversa $f^{-1}$ é suave.

Definição 1.2 : Um subconjunto $M \subset \mathbb{R}^{k}$ é uma $m$-variedade suave em $\mathbb{R}^{k}$ (ou superfície $m$-dimensional em $\mathbb{R}^{k}$ ) se, para cada $x \in M$, existe uma vizinhança $W \cap M$, 
com $W$ uma vizinhança de $x$ em $\mathbb{R}^{k}$, que é difeomorfa a algum aberto $U \subset \mathbb{R}^{m}$. Neste caso, dizemos que o difeomorfismo $g: U \rightarrow W \cap M$ é uma parametrização $e$ $g^{-1}: W \cap M \rightarrow U$ é um sistema de coordenadas.

Definição 1.3 : Seja $H^{m}$ o subespaço de $\mathbb{R}^{m}$ consistindo dos pontos

$$
H^{m}=\left\{\left(x_{1}, \ldots, x_{m}\right) \in \mathbb{R}^{m} ; x_{m} \geq 0\right\} .
$$

Uma $m$-variedade com bordo é um conjunto $X \subset \mathbb{R}^{n}$ tal que cada ponto $x \in X$ possui uma vizinhança $W$ em $\mathbb{R}^{n}$ tal que $W \cap M$ é difeomorfa à uma vizinhança do $\mathbb{R}^{m}$ ou a $H^{m}$. O bordo de $X$ (denotado por $\partial X$ ) consiste dos pontos que não têm vizinhança homeomorfa a um aberto do $\mathbb{R}^{m}$.

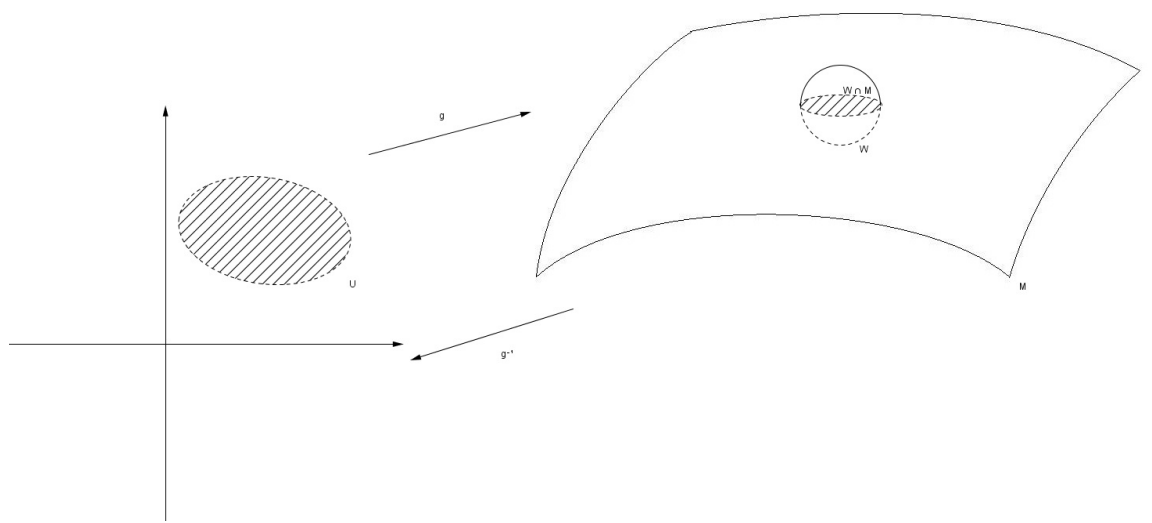

Figura 1.1: Difeomorfismo $g$

\section{Exemplo 1.4:}

i. $X \subset \mathbb{R}^{k}$ é uma 0 -variedade conexa se, e somente se, $X$ é ponto isolado.

ii. A esfera unitária $S^{2}=\left\{(x, y, z) \in \mathbb{R}^{3} ; x^{2}+y^{2}+z^{2}=1\right\}$ é uma 2 - variedade suave. Com efeito, basta considerarmos os seguintes difeomorfismos definidos no disco aberto unitário em $\mathbb{R}^{2}$ : 


$$
\left\{\begin{array}{c}
\phi_{1}(x, y)=\left(x, y, \sqrt{1-x^{2}-y^{2}}\right) \\
\phi_{2}(x, y)=\left(x, y,-\sqrt{1-x^{2}-y^{2}}\right) \\
\phi_{3}(x, z)=\left(x, \sqrt{1-x^{2}-z^{2}}, z\right) \\
\phi_{4}(x, z)=\left(x,-\sqrt{1-x^{2}-z^{2}}, z\right) \\
\phi_{5}(y, z)=\left(\sqrt{1-y^{2}-z^{2}}, y, z\right) \\
\phi_{6}(y, z)=\left(-\sqrt{1-y^{2}-z^{2}}, y, z\right) .
\end{array}\right.
$$

Para definir a noção de derivada de uma aplicação suave $f: M^{m} \rightarrow N^{n}$ entre duas variedades $M$ e $N$, dado $x \in M \subset \mathbb{R}^{k}$, vamos associar um espaço vetorial $T_{x} M \subset \mathbb{R}^{k}$ de dimensão $m$, chamado espaço tangente à $M$ em $x$. A aplicação $d f_{x}$ será uma aplicação linear de $T_{x} M$ em $T_{y} N$, onde $y=f(x)$. Intuitivamente, $T_{x} M$ será o $m$-espaço linear que melhor aproxima $M$ em $x$, quando transladado da origem para $x$.

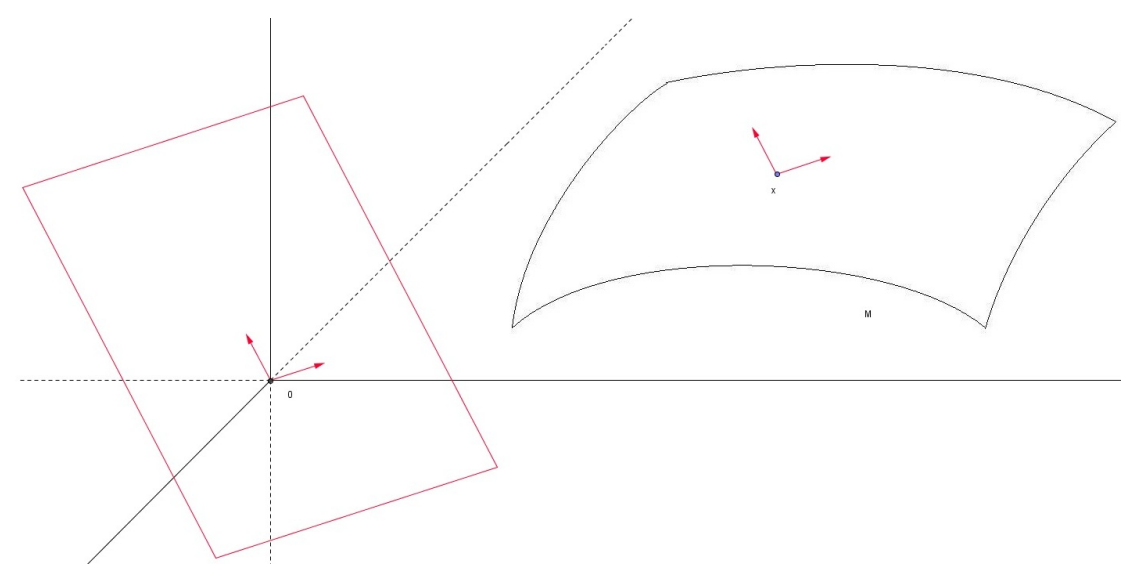

Figura 1.2: $T_{x} M$

Definição 1.5 : O espaço vetorial tangente à superfície $m$-dimensional $M \subset \mathbb{R}^{n}$ no ponto $p \in M$ é o conjunto $T_{p} M$ formado por todos os vetores $\lambda^{\prime}(0)$, onde $\lambda:(-\epsilon, \epsilon) \subset$ $\mathbb{R} \rightarrow M$ é um caminho diferenciável, com $\lambda(0)=p$. Temos que $T_{p} M$ é um subespaço vetorial de $\mathbb{R}^{n}$ com base $\left\{\frac{\partial \phi}{\partial x_{1}}\left(x_{0}\right), \ldots, \frac{\partial \phi}{\partial x_{m}}\left(x_{0}\right)\right\}$, onde $\phi$ é uma parametrização de uma vizinhança $V$ de $p, \operatorname{com} \phi\left(x_{0}\right)=p$.

Observação 1.6 : Seja $U \subset \mathbb{R}^{k}$ um aberto. Temos que $T_{x} U=\mathbb{R}^{k}$ e isto segue do fato do difeomorfismo em questão ser a aplicação identidade. 
Observação 1.7 : Se $f: U \rightarrow V$ é uma aplicação entre os abertos $U \subset \mathbb{R}^{k}$ e $V \subset \mathbb{R}^{l}$, então $d f_{x}: \mathbb{R}^{k} \rightarrow \mathbb{R}^{l}$ é dada por

$$
d f_{x}(h)=\lim _{t \rightarrow 0} \frac{f(x+t h)-f(x)}{t}, \quad x \in U, h \in \mathbb{R}^{k}
$$

Temos que $d f_{x}(h)$ é a aplicação linear na variável $h$ associada à matriz $\left(\frac{\partial f_{i}}{\partial x_{j}}\right)$ das derivadas parciais calculadas em $x$.

A aplicação derivada possui as seguintes propriedades:

1. Regra da Cadeia: Se $f: U \rightarrow V$ e $g: V \rightarrow W$ são suaves, com $y=f(x)$, então $d(g \circ f)_{x}=d g_{y} \circ d f_{x}$. Isto é,

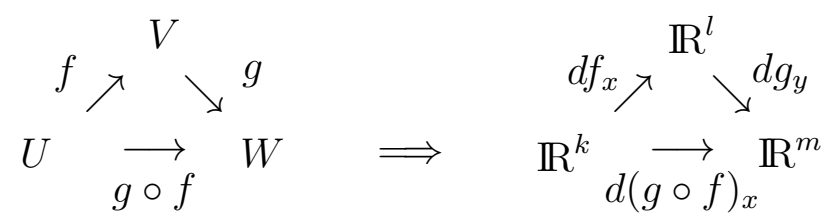

2. Se $U \subset U^{\prime} \subset \mathbb{R}^{k}$ são abertos, então a aplicação inclusão $i: U \rightarrow U^{\prime}$ induz a aplicação identidade $d i_{x}$ do $\mathbb{R}^{k}, \forall x \in U$.

3. Teorema da Aplicação Inversa: Se $d f_{x}: \mathbb{R}^{k} \rightarrow \mathbb{R}^{k}$ é não singular, então $f$ é um difeomorfismo de uma vizinhança aberta $U$ de $x$ em um aberto $f(U)$.

A condição acima não implica a injetividade global de $f$, como vemos no próximo exemplo:

Exemplo 1.8 Seja $f(t)=\left(t^{3}-t, t^{2}\right)$. Temos que $f^{\prime}(t)=\left(3 t^{2}-1,2 t\right)$, assim,

$$
f^{\prime}(t)=0 \leftrightarrow\left\{\begin{array}{c}
3 t^{2}=1 \\
2 t=0
\end{array}\right.
$$

Portanto, $f^{\prime}(t) \neq 0, \forall t \in \mathbb{R}$. Entretanto, $f(-1)=(0,1)=f(1)$, logo $f$ não é injetora. 
Definição 1.9 : Seja $f: M \rightarrow \mathbb{R}$ uma função $C^{\infty}$ definida em uma superfície compacta $M$. Diz-se que $p \in M$ é um ponto crítico da função $f$ quando para alguma (portanto, para qualquer) parametrização $\phi: V_{0} \subset \mathbb{R}^{m} \rightarrow V \subset M$, com $\phi\left(x_{0}\right)=p e$ $V_{0}$ aberto em $\mathbb{R}^{m}$, a função $f \circ \phi: V_{0} \rightarrow \mathbb{R}$ tem todas as derivadas parciais iguais a zero em $x_{0} \in V_{0}$. Dizemos ainda que o ponto crítico $p$ é não degenerado se a matriz Hessiana

$$
H(f)=\left(\frac{\partial^{2}(f \circ \phi)}{\partial x_{i} \partial x_{j}}\left(x_{0}\right)\right)=\left(\begin{array}{cccc}
\frac{\partial^{2}(f \circ \phi)}{\partial x_{1}^{2}}\left(x_{0}\right) & \frac{\partial^{2}(f \circ \phi)}{\partial x_{1} \partial x_{2}}\left(x_{0}\right) & \ldots & \frac{\partial^{2}(f \circ \phi)}{\partial x_{1} \partial x_{m}}\left(x_{0}\right) \\
\vdots & \vdots & \ldots & \vdots \\
\frac{\partial^{2}(f \circ \phi)}{\partial x_{m} \partial x_{1}}\left(x_{0}\right) & \frac{\partial^{2}(f \circ \phi)}{\partial x_{m} \partial x_{2}}\left(x_{0}\right) & \ldots & \frac{\partial^{2}(f \circ \phi)}{\partial x_{m}^{2}}\left(x_{0}\right)
\end{array}\right)
$$

tem determinante não nulo.

Observação 1.10 : A definição de ponto crítico não degenerado não depende da parametrização $\phi$, isto é, o determinante de $H(f)$ ser diferente de zero não depende da parametrização $\phi$ escolhida.

Observação 1.11 : Como $f$ e $\phi$ são aplicações $C^{\infty}$, temos que $f \circ \phi$ também é $C^{\infty}$. Assim, podemos aplicar o Teorema de Schwartz, que nos diz que a matriz $H(f)$ é simétrica, logo seus autovalores são reais.

Definição 1.12 : O número de autovalores negativos da matriz Hessiana $H(f)$ é chamado indice de Morse do ponto crítico $p \in M$.

Os pontos críticos de índice zero são os pontos de mínimo relativo de f, enquanto os de índice $m(m=\operatorname{dim} M)$ são os pontos de máximo relativo de $f$. Os demais pontos são pontos de sela.

Definição 1.13 : Seja $M$ uma superfície compacta. Se $f: M \rightarrow \mathbb{R}$ tem apenas pontos críticos não degenerados, então f é uma função de Morse. 
Exemplo 1.14 : Seja $S^{2}$ a esfera. Tomemos as parametrizações das calotas superior e inferior respectivamente

$$
\phi_{1}(x, y)=\left(x, y,\left(1-x^{2}-y^{2}\right)^{\frac{1}{2}}\right) \quad \phi_{2}(x, y)=-\left(x, y,\left(1-x^{2}-y^{2}\right)^{\frac{1}{2}}\right) .
$$

Seja $f: S^{2} \rightarrow \mathbb{R}$ dada por $f(x, y, z)=z$ (função altura). Temos então que

$$
f \circ \phi_{1}(x, y)=\left(1-x^{2}-y^{2}\right)^{\frac{1}{2}} \quad f \circ \phi_{2}(x, y)=-\left(1-x^{2}-y^{2}\right)^{\frac{1}{2}}
$$

Derivando com relação a $x$ e a y obtemos

$$
\begin{array}{ll}
\frac{\partial\left(f \circ \phi_{1}\right)}{\partial x}(x, y)=\frac{-x}{\sqrt{1-x^{2}-y^{2}}} & \frac{\partial\left(f \circ \phi_{1}\right)}{\partial y}(x, y)=\frac{-y}{\sqrt{1-x^{2}-y^{2}}} \\
\frac{\partial\left(f \circ \phi_{2}\right)}{\partial x}(x, y)=\frac{x}{\sqrt{1-x^{2}-y^{2}}} & \frac{\partial\left(f \circ \phi_{2}\right)}{\partial y}(x, y)=\frac{y}{\sqrt{1-x^{2}-y^{2}}}
\end{array}
$$

Logo, temos que os pontos críticos de $f$ são $(x, y, z)=(0,0,1)$ para $\phi_{1}$ e $(x, y, z)=$ $(0,0,-1)$ para $\phi_{2}$. Calculando as derivadas de segunda ordem de $f \circ \phi_{1}$, temos

$$
\begin{aligned}
& \frac{\partial^{2}\left(f \circ \phi_{1}\right)}{\partial x^{2}}(x, y)=-\frac{x^{2}}{\left(1-x^{2}-y^{2}\right)^{3 / 2}}-\frac{1}{\sqrt{1-x^{2}-y^{2}}} \quad \frac{\partial^{2}\left(f \circ \phi_{1}\right)}{\partial x \partial y}(x, y)=-\frac{y x}{\left(1-x^{2}-y^{2}\right)^{3 / 2}} \\
& \frac{\partial^{2}\left(f \circ \phi_{1}\right)}{\partial y \partial x}(x, y)=-\frac{y x}{\left(1-x^{2}-y^{2}\right)^{3 / 2}} \quad \frac{\partial^{2}\left(f \circ \phi_{1}\right)}{\partial y^{2}}(x, y)=-\frac{y^{2}}{\left(1-x^{2}-y^{2}\right)^{3 / 2}}-\frac{1}{\sqrt{1-x^{2}-y^{2}}}
\end{aligned}
$$

Análogo para $f \circ \phi_{2}$, mudando apenas os sinais. Denotemos por $H_{1}(f)$ e $H_{2}(f)$ as matrizes Hessianas de $f$ relativas às parametrizações $\phi_{1}$ e $\phi_{2}$ respectivamente. Assim,

$$
H_{1}(f)=\left(\begin{array}{cc}
-1 & 0 \\
0 & -1
\end{array}\right) \Rightarrow \lambda_{1}=\lambda_{2}=-1 \quad H_{2}(f)=\left(\begin{array}{cc}
1 & 0 \\
0 & 1
\end{array}\right) \Rightarrow \lambda_{1}=\lambda_{2}=1
$$

Logo, o ponto crítico $(0,0,1)$ tem 2 autovalores negativos e o ponto crítico $(0,0,-1)$ 
não possui autovalor negativo, isto é, estes pontos têm índice de Morse 2 e 0 respectivamente. Como $\operatorname{dim}\left(S^{2}\right)=2$, temos que $(0,0,1)$ é ponto de máximo local e $(0,0,-1)$ é ponto de mínimo local.

Teorema 1.15: (Função Implícita) Seja $f: \mathbb{R}^{k} \times \mathbb{R}^{m} \rightarrow \mathbb{R}^{m}$ uma função suave. Suponha que $f\left(x_{0}, y_{0}\right)=0$ e

$$
\operatorname{det}\left[\frac{\partial f}{\partial y}\left(x_{0}, y_{0}\right)\right] \neq 0
$$

Então, existe um aberto $W \subset \mathbb{R}^{k}$ e uma função suave $F: W \rightarrow \mathbb{R}^{k}$ tais que

i. $x_{0} \in W$ e $F\left(x_{0}\right)=y_{0}$.

ii. $f(x, F(x))=0, \forall x \in W$.

\subsection{Orientação de Variedades}

Definição 1.16 : Uma orientação para um espaço vetorial real de dimensão $m$ é uma classe de equivalência para as bases, da seguinte maneira: duas bases têm a mesma orientação se a matriz de mudança de bases tem determinante positivo, e orientação contrária se o determinante é negativo. Uma variedade suave orientada consiste de uma variedade suave $M$ com a escolha de uma base para cada espaço tangente $T_{x} M$. Se $m \geq 1$, então, para cada ponto existe uma vizinhança $U \subset M$ que é difeomorfa a um aberto em $\mathbb{R}^{m}$ ou em $H^{m}$ (semiespaço superior com o eixo das abscissas), vamos fixar a orientação positiva quando o difeomorfismo preserva a orientação da base canônica do $\mathbb{R}^{m}$, isto é, quando a matriz mudança de bases correspondente à transformação linear da derivada do difeomorfismo tiver determinante positivo.

Se $M$ é compacta e conexa, então $M$ tem exatamente duas possíveis orientações. Caso $M$ seja uma variedade com bordo, temos três tipos de vetores no espaço tangente em cada ponto do bordo, da seguinte forma: 
- vetores tangentes ao bordo, formando um subespaço $(m-1)-\operatorname{dimensional~} T_{x}(\partial M) \subset$ $T_{x} M$,

- vetores que apontam para fora, formando metade de um espaço aberto limitado por $T_{x}(\partial M)$,

- vetores que apontam para dentro, formando a metade complementar desse espaço.

Quando a variedade tem dimensão um, escolhemos a orientação +1 ou -1 , dependendo se o vetor aponta para fora ou para dentro da variedade, respectivamente.

\subsection{Homologia Simplicial}

Definição 1.17 : Um simplexo $s$ de dimensão $m$ em $\mathbb{R}^{n}(n \geq m)$ é determinado por $m+1$ vértices $a_{0}, \ldots, a_{m} \in \mathbb{R}^{n}$, tais que os vetores $a_{1}-a_{0}, a_{2}-a_{0}, \ldots, a_{m}-a_{0}$ são linearmente independentes. O simplexo $s=\left[a_{0}, \ldots, a_{m}\right]$ é o conjunto de todas as combinações lineares convexas $\sum_{i=1}^{m} t_{i} \cdot a_{i}$, com $t_{i} \geq 0 e \sum_{i=1}^{m} t_{i}=1$. Equivalentemente, $s$ é o menor convexo que contém $a_{0}, \ldots, a_{m} \in \mathbb{R}^{n}$.

Exemplo 1.18 : Em $\mathbb{R}^{3}$, considere os vértices $a_{0}=(0,0,0), a_{1}=(1,0,0), a_{2}=$ $(0,1,0)$ e $a_{3}=(0,0,1)$. O simplexo $s=\left[a_{0}, a_{1}, a_{2}, a_{3}\right]$ será o tetraedro, como na figura 1.3 .

Definição 1.19 : Uma face de dimensão $k$ de um simplexo $s=\left[a_{0}, \ldots, a_{m}\right]$ é um simplexo $t=\left[a_{i_{0}}, \ldots, a_{i_{k}}\right]$, com $a_{i_{0}}, \ldots, a_{i_{k}} \in\left\{a_{0}, \ldots, a_{m}\right\}$.

Exemplo 1.20 : Se tomarmos o simplexo s do Exemplo 1.18, s possui 4 faces de dimensão 0, 6 faces de dimensão 1, 4 faces de dimensão 2 e 1 face de dimensão 3.

Definição 1.21 : Um poliedro $P$ em $\mathbb{R}^{n}$, de dimensão $m(m \leq n)$, é uma coleção finita de simplexos em $\mathbb{R}^{n}$, tais que 


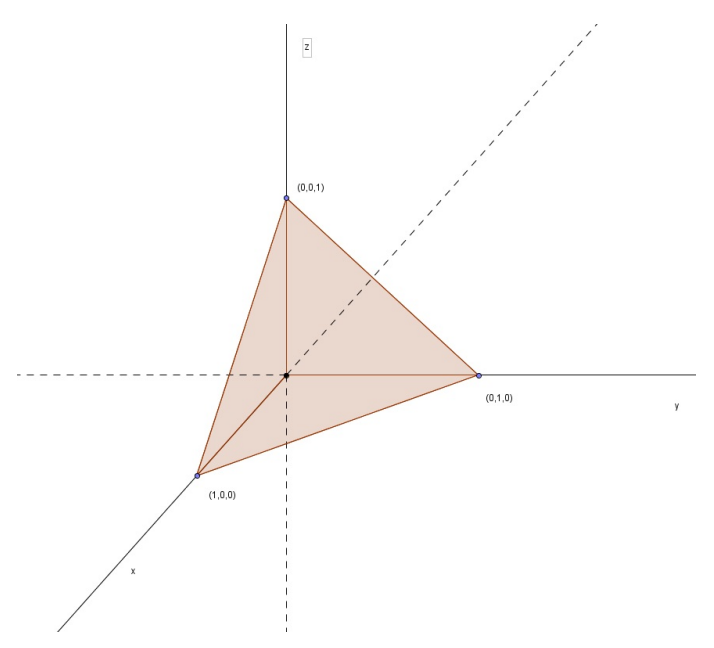

Figura 1.3: Simplexo $s$

i. Se s é um simplexo de $P$, então toda face de s é um simplexo de $P$

ii. Se $s^{\prime}$ e $s^{\prime \prime}$ são simplexos de $P$ então ou $s^{\prime} \cap s^{\prime \prime}$ é uma face em comum ou $s^{\prime} \cap s^{\prime \prime}$ é vazio.

iii. A maior dimensão entre os simplexos é $m$.

Indicaremos por $|P|$ a reunião dos simplexos de $P$. Note que conhecer $|P|$ não significa conhecer os simplexos de $P$.

Exemplo 1.22 : Seja s o simplexo do Exemplo ??. Considere o poliedro $P$ das faces do simplexo s. Sejam

$$
\begin{aligned}
& s^{\prime}=\left[(0,0,0),(0,0,1),(1,0,0),\left(\frac{1}{2}, \frac{1}{2}, 0\right)\right], \quad \text { simplexos em } \mathbb{R}^{3} \\
& s^{\prime \prime}=\left[(0,0,0),(0,0,1),(0,1,0),\left(\frac{1}{2}, \frac{1}{2}, 0\right)\right]
\end{aligned}
$$

Defina $P^{\prime}$ como o poliedro formado pelas faces dos simplexos $s^{\prime}$ e $s^{\prime \prime}$. Assim, temos que $|P|=\left|P^{\prime}\right|$, porém seus simplexos são diferentes.

Definição 1.23 : Dado um poliedro $P$ de dimensão $m$, para cada $i=0,1, \ldots, m$, defina 
$\alpha_{i}$ o número de simplexos de dimensão $i$ em $P$. O número

$$
\chi(P)=\sum_{i=0}^{m}(-1)^{i} \alpha_{i}
$$

é chamado característica de Euler-Poincaré do poliedro P.

Exemplo 1.24 : Note que se $P$ é um poliedro formado pelas faces de um simplexo $m$-dimensional s, então

$$
\alpha_{i}=\left(\begin{array}{c}
m+1 \\
i+1
\end{array}\right) .
$$

Isto se deve ao fato de que, para se obter uma face de dimensão $i$, basta escolher $i+1$ vértices dentre os $m+1$. Além disso, observe que

$$
\begin{aligned}
(1+(-1))^{m+1} & =\sum_{i=0}^{m+1}\left(\begin{array}{c}
m+1 \\
i
\end{array}\right) \cdot 1^{m+1-i} \cdot(-1)^{i} \\
& =1+\sum_{i=1}^{m+1}(-1)^{i}\left(\begin{array}{c}
m+1 \\
i
\end{array}\right)=1-\sum_{i=0}^{m}\left(\begin{array}{c}
m+1 \\
i+1
\end{array}\right)(-1)^{i}=0 .
\end{aligned}
$$

Portanto, de (1) e de (2), segue que $\chi(P)=1$. Assim, se $Q$ é o poliedro formado pelas faces de dimensão $\leq m$ de um simplexo de dimensão $m+1$, temos que $\chi(Q)=$ $1+(-1)^{m}$. De fato, se $P$ é o poliedro formado pelas faces de dimensão $\leq m+1$ de um simplexo de dimensão $m+1$, temos que

$$
\begin{gathered}
\chi(P)=\sum_{i=0}^{m+1}\left(\begin{array}{c}
m+2 \\
i+1
\end{array}\right)(-1)^{i}=(-1)^{m+1}+\underbrace{\sum_{i=0}^{m}\left(\begin{array}{c}
m+2 \\
i+1
\end{array}\right)(-1)^{i}}_{=\chi_{(Q)}}=1 \\
\Rightarrow \chi(Q)=1-(-1)^{m+1}=1+(-1)^{m} .
\end{gathered}
$$

Além disso, temos que $Q$ é homeomorfo a esfera $S^{m}$ (ver [14]) e que a característica de Euler-Poincaré é um invariante topológico, logo $\chi\left(S^{m}\right)=1+(-1)^{m}$. Assim, temos que $\chi\left(S^{m}\right)=2$ se $m$ é par e $\chi\left(S^{m}\right)=0$ se $m$ é ímpar.

Definição 1.25 : Para cada $i=0,1, \ldots, m$ uma cadeia de dimensão $i$ no poliedro 
$P$ é um conjunto $C=\left\{s_{1}, \ldots, s_{k}\right\}$ cujos elementos são simplexos de dimensão $i$ em P. Estamos considerando também a cadeia vazia, que denotaremos por 0. Para cada simplexo s de dimensão $i$ em $P$, a cadeia $\{s\}$ será denotada simplesmente por $s$.

Definição 1.26 : Seja $C_{i}=C_{i}(P)$ o conjunto das cadeias de dimensão i em P. Dadas duas cadeias $c_{1}, c_{2} \in C_{i}$, definimos a soma $c_{1}+c_{2}=\left(c_{1} \cup c_{2}\right)-\left(c_{1} \cap c_{2}\right)$.

Observação 1.27 : O conjunto $C_{i}$ com esta operação é um grupo abeliano. Segue daí, que $C_{i}$ é um espaço vetorial sobre o corpo $\mathbb{Z}_{2}$. Deste modo, uma cadeia $C=\left\{s_{1}, \ldots, s_{k}\right\}$ pode ser escrita como $C=s_{1}+\ldots+s_{k}$ e, assim, os simplexos de dimensão $i$ em $P$ formam uma base para $C_{i}$. Em particular, $\operatorname{dim} C_{i}=\alpha_{i}$.

Definição 1.28 : Para cada $i=0,1, \ldots$, m definimos o homomorfismo de bordo como a transformação linear

$$
\partial_{i}: C_{i} \rightarrow C_{i-1}
$$

tal que, para cada simplexo $s \in C_{i}$, $\partial_{i} s$ é igual ao conjunto das faces de dimensão $i-1$ do simplexo s. Por conveniência, adotaremos que $C_{-1}=0$, assim, $\partial_{0}=0$.

Note que se $s \in C_{i}$ então cada face de dimensão $i-2$ está contida exatamente em duas faces de dimensão $i-1$, o que nos dá

$$
\partial_{i-1} \circ \partial_{i}(s)=0
$$

visto que o inverso de cada elemento é ele próprio.

Sejam $Z_{i} \subset C_{i}$ o núcleo de $\partial_{i}$ e $B_{i} \subset C_{i}$ a imagem de $\partial_{i+1}$. Os elementos em $Z_{i}$ são chamados ciclos de dimensão $i$ e os elementos em $B_{i}$ são chamados bordos de dimensão i. A relação fundamental $\partial_{i-1} \circ \partial_{i}=0$ nos diz que $B_{i} \subset Z_{i}$, isto é, todo bordo é um ciclo. Sejam $z_{i}=\operatorname{dim} Z_{i}$ e $b_{i}=\operatorname{dim} B_{i}$. O Teorema do Núcleo e Imagem nos diz que a dimensão do domínio de uma transformação linear é igual a dimensão do núcleo mais a dimensão da imagem, logo, $\alpha_{i}=z_{i}+b_{i-1}\left(\right.$ note que $\left.b_{m}=b_{-1}=0\right)$. 
Definição 1.29 O espaço vetorial quociente

$$
H_{i}(P)=\frac{Z_{i}}{B_{i}}
$$

chama-se $i$-ésimo grupo de homologia do poliedro $P$ e a dimensão desse espaço, $\beta_{i}=$ $z_{i}-b_{i}$, é chamado i-ésimo número de Betti módulo 2 do poliedro P.

Teorema 1.30 : (Poincaré) Sejam $\beta_{0}, \beta_{1}, \ldots, \beta_{m}$ os números de Betti do poliedro $P$, então vale a igualdade:

$$
\chi(P)=\beta_{0}-\beta_{1}+\ldots+(-1)^{m} \cdot \beta_{m}=\sum_{i=0}^{m}(-1)^{i} \cdot \beta_{i} .
$$

\section{Demonstração:}

$$
\chi(P)=\sum_{i=0}^{m}(-1)^{i} \cdot \alpha_{i}=\sum_{i=0}^{m}(-1)^{m} \cdot\left(z_{i}+b_{i-1}\right)=\sum_{i=0}^{m}(-1)^{i} \cdot\left(z_{i}-b_{i}\right)=\sum_{m}^{i=0}(-1)^{i} \cdot \beta_{i},
$$

donde a terceira igualdade ocorre do fato de que $b_{m}=b_{-1}=0$.

\section{Desigualdades de Morse}

Seja $f: M \rightarrow \mathbb{R}$ uma função de Morse. Para cada $i=1, \ldots, m(m=\operatorname{dim}(M))$ sejam $M_{i}$ os números de pontos críticos de $f$ com índice $i$ e $\beta_{i}$ o número de Betti de dimensão $i$ de $M$. Então, as desigualdades de Morse são as seguintes

$$
\begin{array}{r}
\beta_{i}-\beta_{i-1}+\ldots \pm \beta_{0} \leq M_{i}-M_{i-1}+\ldots \pm M_{0} \\
\beta_{m}-\beta_{m-1}+\ldots \pm \beta_{0}=M_{m}-M_{m-1}+\ldots \pm M_{0}
\end{array}
$$

Assim, da igualdade acima, obtemos que

$$
\sum_{i=1}^{m}(-1)^{i} M_{i}=\chi(M)
$$


Note que, se somarmos as duas primeiras desigualdades, obtemos

$$
+\left\{\begin{array}{rl}
\beta_{1}-\beta_{0} & \leq M_{1}-M_{0} \\
\beta_{2}-\beta_{1}+\beta_{0} & \leq M_{2}-M_{1}+M_{0}
\end{array} \quad \Rightarrow \quad \beta_{2} \leq M_{2} .\right.
$$

Procedendo deste modo, somando a i-ésima desigualdade de Morse com a anterior, obtemos

$$
+\left\{\begin{array}{l}
\beta_{i}-\beta_{i-1}+\ldots \pm \beta_{0} \leq M_{i}-M_{i-1}+\ldots \pm M_{0} \\
\beta_{i+1}-\beta_{i}+\ldots \mp \beta_{0} \leq M_{i+1}-M_{i}+\ldots \mp M_{0}
\end{array} \quad \Rightarrow \quad \beta_{i+1} \leq M_{i+1}\right.
$$

Temos que a igualdade $(*)$ decorre do Teorema de Poincaré-Hopf 2.22 , De fato, se $f: M \rightarrow \mathbb{R}$ é uma função de Morse, pode-se provar que $\nabla f$ é um campo de vetores tangentes à $M$ cujas singularidades são os pontos críticos de $f$. Em cada ponto crítico, o índice da singularidade é igual a $(-1)^{i}$, onde $i$ é o índice de Morse do ponto crítico $p$.

\subsection{Introdução à Teoria de Singularidades}

Nosso objetivo é estudar aplicações $f: \mathbb{K}^{n} \rightarrow \mathbb{K}^{p}, \mathbb{K}=\mathbb{R}$ ou $\mathbb{C}$, na vizinhança de um ponto singular.

Definição 1.31 : Sejam $f_{1}: U_{1} \rightarrow \mathbb{K}^{p}, f_{2}: U_{2} \rightarrow \mathbb{K}^{p}$ e $x \in U_{1} \cap U_{2} \subset \mathbb{K}^{n}$, com $U_{1}$ e $U_{2}$ abertos. Dizemos que $f_{1} \backsim f_{2}$ ( $f_{1}$ está relacionada com $f_{2}$ ), se existe uma vizinhança $U \subset \mathbb{K}^{n}$ com $x \in U$ tal que $\left.f_{1}\right|_{U}=\left.f_{2}\right|_{U}$.

Observação 1.32 A relação $\backsim$ é uma relação de equivalência pois
i. $f_{1} \backsim f_{1}$;
ii. $f_{1} \backsim f_{2} \Rightarrow f_{2} \backsim f_{1}$;
iii. $f_{1} \backsim f_{2}$ e $f_{2} \backsim f_{3} \Rightarrow f_{1} \backsim f_{3}$. 
Denominamos germe suave (analítico) de uma aplicação de $\mathbb{K}^{n}$ em $\mathbb{K}^{p}$ no ponto $x$ à classe de equivalência definida acima. É usual adotarmos a notação $f:\left(\mathbb{K}^{n}, x\right) \rightarrow$ $\left(\mathbb{K}^{p}, y\right)$ para o germe definido acima, além disso, geralmente chamamos $\left(\mathbb{K}^{n}, x\right)$ de "fonte"e $\left(\mathbb{K}^{p}, y\right)$ de "meta".

Quando $\mathbb{K}=\mathbb{R}$, indicaremos por $\varepsilon_{n, p}$ o conjunto de todos os germes $f: \mathbb{K}^{n} \rightarrow \mathbb{K}^{p}$. Quando $\mathbb{K}=\mathbb{C}$, indicaremos por $\mathcal{O}_{n, p}$ o mesmo conjunto. No caso $p=1$ é comum denotarmos tais conjuntos apenas por $\varepsilon_{n}$ e $\mathcal{O}_{n}$.

Definição 1.33 : Vamos denotar por $\mathfrak{M}_{n}$ o subconjunto de $\varepsilon_{n}$ de todos os germes que tem meta 0 .

Definição 1.34 : Um ideal I de um anel $(A,+, \cdot)$ comutativo com identidade é dito maximal se $\forall B \subset A$ ideal, com $I \subset B \subset A$, têm-se que $B=I$ ou $B=A$, e além disso, $I \neq A$.

Proposição 1.35 : O ideal $\mathfrak{M}_{n}$ é o único ideal maximal de $\varepsilon_{n}$.

Demonstração: Suponha que exista $I \subset \varepsilon_{n}$ tal que $\mathfrak{M}_{n} \subsetneq I \subset \varepsilon_{n}$. Logo, existe algum $f \in I$ tal que $f$ é invertível. Portanto, $I$ não é maximal pois $I=\varepsilon_{n}$.

Definição 1.36 : Um anel local é um anel com um único ideal maximal.

Os seguintes resultados podem ser encontrados em [6]

Proposição 1.37 : O ideal $\mathfrak{M}_{n}^{k}$ é gerado por todos os monômios nas variáveis $x_{1}, \ldots, x_{n}$ de grau igual a $k$.

Proposição 1.38 (Lema de Nakayama): Seja $\varepsilon$ um anel comutativo com identidade, e seja $\mathfrak{M}$ um ideal em $\varepsilon$ com a propriedade de que $1+x$ é invertivel em $\varepsilon$ para todo $x \in \mathfrak{M}$. Além disso, seja $M$ um $\varepsilon$-módulo e $A, B \subset M$ E-submódulos com A finitamente gerado. Se $A \subset B+\mathfrak{M} A$, então $A \subset B$. 
Demonstração: Sejam $a_{1}, \ldots, a_{t}$ geradores de $A$. Pela hipótese podemos encontrar $b_{1}, \ldots, b_{t} \in B$ e $\lambda_{i j} \in \mathfrak{M}$ com $1 \leq i \leq t$ tais que

$$
a_{i}=b_{i}+\lambda_{i 1} \cdot a_{1}+\ldots+\lambda_{i t} a_{t} .
$$

Sendo $\Lambda=\left(\lambda_{i j}\right)$ uma matriz $t \times t$ e denotando $a=\left(a_{1}, \ldots, a_{t}\right)$ e $b=\left(b_{1}, \ldots, b_{t}\right)$, podemos reescrever (1) como

$$
(I-\Lambda) a=b
$$

onde $I$ é a matriz identidade $t \times t$ sobre o anel $\varepsilon$. Afim de que possamos escrever $a_{i} \mathrm{em}$ termos de $b_{j}$ com $1 \leq j \leq t$ é suficiente mostrar que $(I-\Lambda)$ é uma matriz invertível. Para isto basta notar que o determinante de $(I-\Lambda)$ é $1-\lambda$ onde $\lambda$ é uma soma de elementos em $\mathfrak{M}$. Segue da hipótese que $1-\lambda \neq 0$, de onde concluímos o resultado.

Definição 1.39 : Sejam $f \in \mathcal{O}_{n}$ um germe de função analítica com singularidade isolada em 0 e $J(f)$ o ideal Jacobiano de $f$. Definimos o número de Milnor $\mu$ do germe $f$ por

$$
\mu=\operatorname{dim}_{\mathbb{C}} \frac{\mathcal{O}_{n}}{J(f)}
$$

As vezes chamaremos o número de Milnor $\mu$ de codimensão de $f$ e denotaremos cod $(f)$. Dizemos ainda que a codimensão de $f$ é finita se cod $(f)$ é finito, do contrário, dizemos que a codimensão de $f$ é infinita.

Mais geralmente, seja $M$ um $\mathcal{O}$ - módulo e $I \subset M$ um $\mathcal{O}$-submódulo. Dizemos que $I$ tem codimensão finita se o espaço quociente $\frac{M}{I}$ tem dimensão finita.

Proposição 1.40 : Sejam $M$ um $\mathcal{O}$-módulo com base finita e $I \subset M$ um $\mathcal{O}$-submódulo. Uma condição necessária e suficiente para que I tenha codimensão finita em $M$ é que exista $k \geq 1$ tal que $\mathfrak{M}^{k} M \subset I$.

Definição 1.41 Suponha o espaço quociente $\frac{I+\mathfrak{M}^{k} M}{I+\mathfrak{M}^{k+1} M}$ de codimensão finita, definimos então

$$
\operatorname{cod}_{k} I=\operatorname{dim} \frac{I+\mathfrak{M}^{k} M}{I+\mathfrak{M}^{k+1} M}, \quad \text { para } k=1,2, \ldots
$$


Proposição 1.42 : Sejam $M$ um $\mathcal{O}$-módulo com base finita e $I \subset M$ um $\mathcal{O}$-submódulo. Uma condição necessária e suficiente para que $I \subset M$ tenha codimensão finita é que para todo $k \geq 1$ tenha-se $\operatorname{cod}_{k} I$ finita e, neste caso,

$$
\operatorname{cod} I=\operatorname{cod}_{0} I+\operatorname{cod}_{1} I+\ldots
$$

Podemos então calcular alguns exemplos de números de Milnor utilizando os resultados enunciados acima.

Exemplo 1.43 : Considere o germe $f(x)=x^{s+1}$ com $s \geq 1$. Temos que Jf $=\left\langle x^{s}\right\rangle$. Para cada $0 \leq k \leq s-1$, temos apenas o monômio $x^{k}$. Então

$$
\begin{aligned}
& \operatorname{cod}_{0} f=1 \\
& \operatorname{cod}_{1} f=\operatorname{dim} \frac{J f+\mathfrak{M}_{n}^{1}}{J f+\mathfrak{M}_{n}^{2}}=1 \\
& \operatorname{cod}_{2} f=\operatorname{dim} \frac{J f+\mathfrak{M}_{n}^{2}}{J f+\mathfrak{M}_{n}^{3}}=1
\end{aligned}
$$

Portanto $\operatorname{cod}(f)=s$.

Exemplo 1.44 : Seja $f(x, y)=x^{2} y+y^{4}$. Temos que

$$
\frac{\partial f}{\partial x}(x, y)=2 x y \quad e \quad \frac{\partial f}{\partial y}(x, y)=x^{2}+4 y^{3}
$$

que se anulam apenas na origem. Vamos computar a codimensão de f. Como $\mathfrak{M}_{2}^{4} \subset$ $J f+\mathfrak{M}_{2}^{5}$ temos que $\operatorname{cod}_{j} f=0, \forall j \geq 4$. Logo, nos resta calcular apenas $\operatorname{cod}_{0} f, \operatorname{cod}_{1} f$, $\operatorname{cod}_{2} f$ e $\operatorname{cod}_{3} f$. Temos

$$
\begin{aligned}
& 1 \\
& x \quad y \\
& \begin{array}{lll}
x^{2} & x y \quad y^{2}
\end{array} \\
& \begin{array}{llll}
x^{3} & x^{2} y & x y^{2} & y^{3}
\end{array} \\
& x^{4} \quad x^{3} y \quad x^{2} y^{2} \quad x y^{3} \quad y^{4} \\
& \begin{array}{llllll}
x^{5} & x^{4} y & x^{3} y^{2} & x^{2} y^{3} & x y^{4} & y^{5}
\end{array}
\end{aligned}
$$


Assim, $\operatorname{cod}_{0} f=1$ pois a constante não pode ser escrita como combinação linear de elementos em $d f$ com elementos de $\mathfrak{M}_{2}^{1}$. Temos $\operatorname{cod}_{1} f=2$ pois $x$ e y não podem ser escritos como combinação linear de elementos em df com elementos de $\mathfrak{M}_{2}^{2}$. Temos $\operatorname{cod}_{2} f=1$ pois $x^{2}=\frac{\partial f}{\partial y}-4 y^{3}$, onde $-4 y^{3} \in \mathfrak{M}_{2}^{3}$ e $x y=\frac{1}{2} \frac{\partial f}{\partial x}$ mas $y^{2}$ não pode ser escrito como combinação linear dos elementos em Jf com os elementos em $\mathfrak{M}_{2}^{3}$. Por fim, temos $x^{3}=x \frac{\partial f}{\partial y}-4 y^{3} x, x^{2} y=\frac{x}{2} \frac{\partial f}{\partial x}$ e $x y^{2}=\frac{y}{2} \frac{\partial f}{\partial x}$ mas $y^{3}$ não pode ser escrito como combinação linear dos elementos em Jf com os elementos em $\mathfrak{M}_{2}^{4}$. Portanto, pela Proposição 1.40 temos

$$
\operatorname{cod}(f)=\operatorname{cod}_{0}(f)+\operatorname{cod}_{1}(f)+\operatorname{cod}_{2}(f)+\operatorname{cod}_{3}(f)+0+0+\ldots=5 .
$$

Exemplo 1.45 : Da mesma forma podemos computar a codimensão de $f(x, y)=$ $x^{3}+x y^{3}$ que é 7 . Neste caso $\operatorname{cod}_{j}(f)=0, \forall j \geq 5$. 


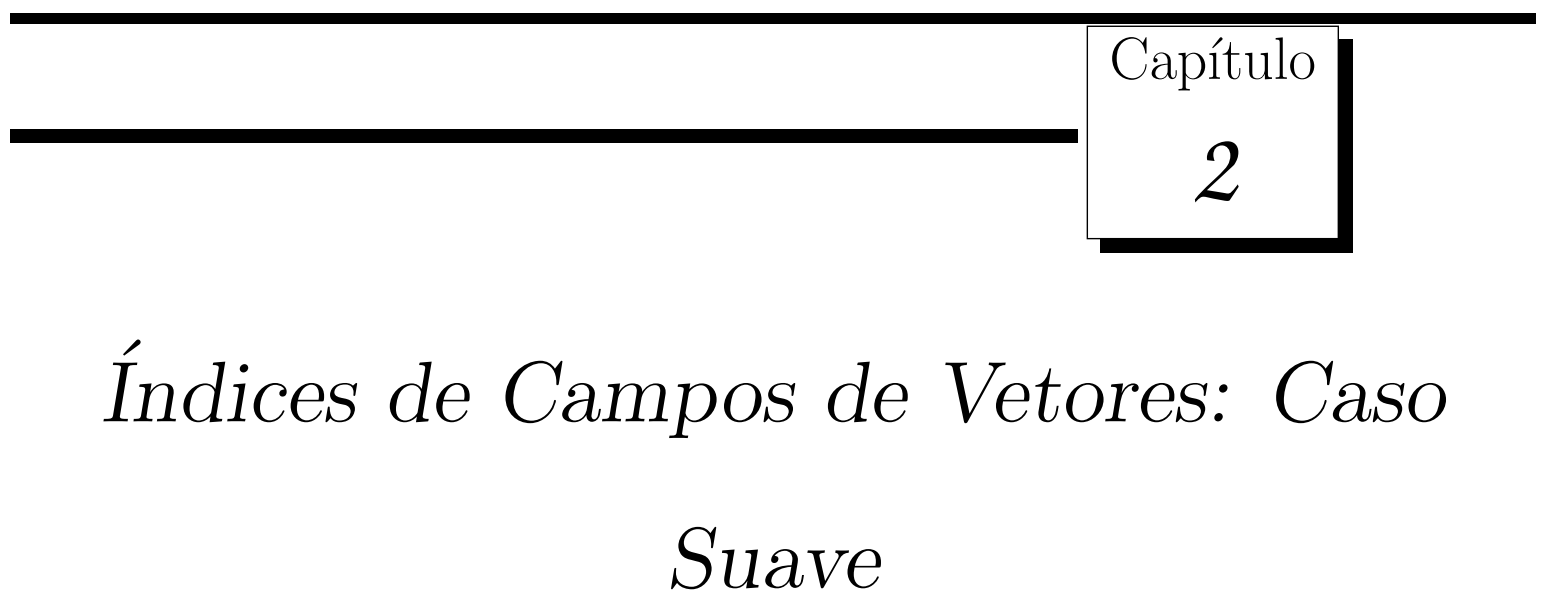

\author{
Suave
}

Neste capítulo veremos a relação do índice de Poincaré-Hopf com a característica de Euler de uma variedade suave. Tal relação é dada pelo teorema de Poincaré-Hopf. Para isto será necessário vermos alguns resultados e definições sobre o grau de uma aplicação.

\title{
2.1 Grau Módulo 2 de uma Aplicação
}

Dado $X \subset \mathbb{R}^{k}$, seja $X \times[0,1]$ o subconjunto de $\mathbb{R}^{k+1}$ de todos os pontos $(x, t)$, com $x \in X$ e $t \in[0,1]$. Duas aplicações

$$
f, g: X \rightarrow Y
$$

são suavemente homotópicas (e denotaremos por $f \sim g$ ) se existe uma aplicação suave $F: X \times[0,1] \rightarrow Y$ com

$$
F(x, 0)=f(x) \quad e \quad F(x, 1)=g(x)
$$

para todo $x \in X$. Esta aplicação $F$ é chamada de homotopia suave entre $f$ e $g$.

Note que a relação de homotopia é uma relação de equivalência. De fato, a única 
propriedade não trivial a ser demonstrada é a transitividade, e esta se procede da seguinte maneira:

Considere a função

$$
\lambda(\tau)=\left\{\begin{array}{r}
0, \text { se } \tau \leq 0 \\
e^{-\frac{1}{\tau}}, \text { se } \tau>0
\end{array}\right.
$$

Defina

$$
\phi(t)=\frac{\lambda\left(t-\frac{1}{3}\right)}{\lambda\left(t-\frac{1}{3}\right)+\lambda\left(\frac{2}{3}-t\right)} .
$$

Deste modo, se $F$ é uma homotopia suave entre $f$ e $g$, temos que $G(x, t)=F(x, \phi(t))$ é uma homotopia suave dada por

$$
G(x, t)=\left\{\begin{array}{l}
f(x), \quad \text { se } t \leq \frac{1}{3} \\
g(x), \text { se } t \geq \frac{2}{3}
\end{array}\right.
$$

Deste modo, sejam $G$ e $H$ homotopias suaves entre $f$ e $g$ e entre $g$ e $h$, definidas como acima, respectivamente. Defina $I(x, t)=G(x, 2 t)$ se $t \leq \frac{1}{2}$ e $I(x, t)=H(x, 2 t-1)$ se $t>\frac{1}{2}$. A aplicação $I$ será a homotopia suave entre as aplicações $f$ e $h$.

Definição 2.1 : Um difeomorfismo $f$ é dito suavemente isotópico a $g$ se existe uma homotopia suave $F$ entre $f$ e $g$ e, além disso, a aplicação $x \mapsto F(x, t)$ é um difeomorfismo para cada $t \in[0,1]$.

Proposição 2.2 : (Lema da Homotopia) Sejam $f, g: M \rightarrow N$ aplicações suavemente homotópicas entre variedades de mesma dimensão, com $M$ compacta e sem bordo. Se $y \in N$ é um valor regular de $f$ e $g$, então

$$
\sharp f^{-1}(y)=\sharp g^{-1}(y)(\bmod 2) \text {. }
$$

Demonstração: Seja $F: M \times[0,1] \rightarrow N$ uma homotopia suave entre as aplicações $f$ e $g$. Primeiramente, suponha que $y$ seja um valor regular de $F$. Então $F^{-1}(y)$ é uma 
1-variedade compacta, suave e com bordo, dada por

$$
F^{-1}(y) \cap(M \times 0 \cup M \times 1)=\left(f^{-1}(y) \times 0\right) \cup\left(g^{-1}(y) \times 1\right) .
$$

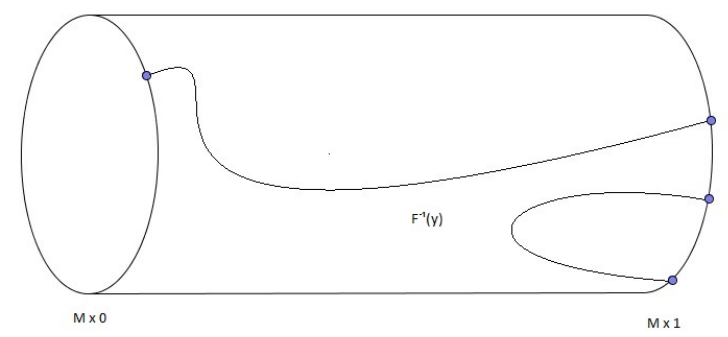

Figura 2.1: $F^{-1}(y)$

Então o número total de pontos de $F^{-1}(y)$ é igual a

$$
\sharp f^{-1}(y)+\sharp g^{-1}(y) \text {. }
$$

Uma 1-variedade compacta tem sempre um número par de pontos de bordo (Ver [20]). Então $\sharp f^{-1}(y)+\sharp g^{-1}(y)$ é par, logo

$$
\sharp f^{-1}(y)=\sharp g^{-1}(y)(\bmod 2) \text {. }
$$

Agora suponha que $y$ não seja um valor regular de $F$. Localmente, temos que $\sharp f^{-1}(y)$ $\mathrm{e} \sharp g^{-1}(y)$ são funções localmente constantes (em uma vizinhança sem pontos críticos). Assim, existe uma vizinhança $V_{1} \subset N$ de $y$, de valores regulares de $f$, tal que

$$
\sharp f^{-1}\left(y^{\prime}\right) \bmod (2)=\sharp f^{-1}(y) \bmod (2), \quad \forall y^{\prime} \in V_{1} .
$$

Da mesma forma, seja $V_{2} \subset N$ uma vizinhança de $y$ de valores regulares de $g$, tal que

$$
\sharp g^{-1}\left(y^{\prime}\right) \bmod (2)=\sharp g^{-1}(y) \bmod (2), \quad \forall y^{\prime} \in V_{2} .
$$


Seja $V=V_{1} \cap V_{2}$ e tome $z \in V$ um valor regular de $F$. Então

$$
\sharp f^{-1}(y) \bmod (2)=\sharp f^{-1}(z) \bmod (2)=\sharp g^{-1}(z) \bmod (2)=\sharp g^{-1}(y) \bmod (2) .
$$

Teorema 2.3 : (Lema da Homogeneidade) Sejam y e z pontos interiores arbitrários de uma variedade $N$ suave e conexa. Então existe um difeomorfismo $h: N \rightarrow N$ que é isotópico a identidade e leva y em z.

Demonstração: Suponha primeiramente $N=S^{n}$. Neste caso basta escolher h rotação que leva $y$ em $z$. Para o caso geral, vamos construir uma isotopia de $\mathbb{R}^{n}$ nele próprio que deixa todos os pontos fora da bola unitária fixos e desliza a origem para um ponto dentro da bola unitária.
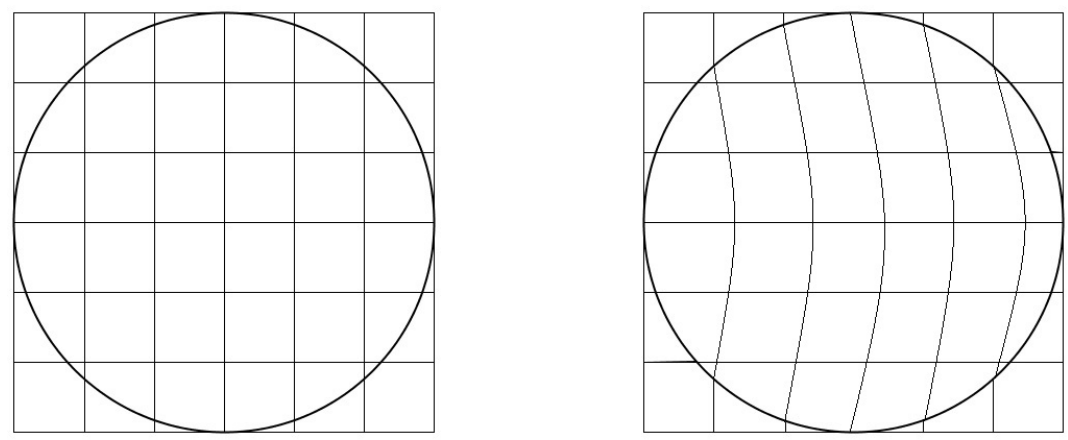

Figura 2.2: Homotopia de $\mathbb{R}^{n}$ em $\mathbb{R}^{n}$

Seja $\phi: \mathbb{R}^{n} \rightarrow \mathbb{R}$ uma função suave que satisfaz

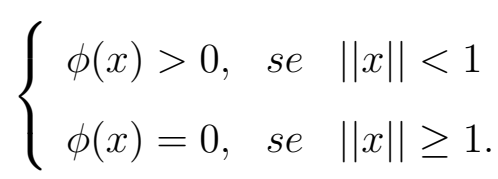

Por exemplo a função $\phi(x)=\lambda\left(1-\|x\|^{2}\right)$, onde $\lambda(t)=0$, para $t \leq 0$ e $\lambda(t)=e^{-t^{-1}}$, para $t>0$. Dado um vetor fixado $c \in S^{n-1}$, considere o sistema de equações diferenciais

$$
\frac{d x_{i}}{d t}=c_{i} \phi\left(x_{1}, \ldots, x_{n}\right), \quad i=1, \ldots, n
$$


Para qualquer $\bar{x} \in \mathbb{R}^{n}$ estas equações tem uma solução única $x=x(t)$, definida em $\mathbb{R}$, satisfazendo a condição inicial $x(0)=\bar{x}$. Vamos usar a notação $x(t)=F_{t}(\bar{x})$ para tal solução. Então, temos

i. $F_{t}(\bar{x})$ está definida para todo $t$ e $F$ depende suavemente de $t$ e $\bar{x}$,

ii. $F_{0}(\bar{x})=\bar{x}$,

iii. $F_{s+t}(\bar{x})=F_{s} \circ F_{t}(\bar{x})$.

Assim, cada $F_{t}$ é um difeomorfismo do $\mathbb{R}^{n}$ nele próprio. Deixando $t$ variar, temos que cada $F_{t}$ é isotópico à identidade por uma isotopia que deixa os pontos fora da bola unitária fixos. Com uma escolha apropriada de $c$, temos que $F_{t}$ deve levar a origem em um ponto interior da bola unitária. Como a isotopia é uma relação de equivalência e, localmente, um ponto sobre $N$ possui uma vizinhança que é difeomorfa a $\mathbb{R}^{n}$, temos que o resultado está provado.

Teorema 2.4 : Seja $f: M \rightarrow N$ uma aplicação suave, com $N$ conexa e $M$ compacta e sem bordo. Se y e z são valores regulares de $f$, então

$$
\sharp f^{-1}(y) \equiv \sharp f^{-1}(z)(\bmod 2) .
$$

Demonstração: Dados dois valores regulares $y$ e $z$ de $f$, considere o difeomorfismo $h$ de $N$ em $N$ que leva $y$ em $z$. Temos que $z$ é um valor regular de $h \circ f$ e, além disso, $h \circ f$ é homotópico à $f$. Assim, pelo Lema da Isotopia, temos

$$
\sharp(h \circ f)^{-1}(z)=\sharp f^{-1}(z)(\bmod 2) .
$$

Além disso,

$$
(h \circ f)^{-1}(z)=f^{-1}\left(h^{-1}(z)\right)=f^{-1}(y) .
$$

Portanto,

$$
\sharp f^{-1}(y) \equiv \sharp f^{-1}(z)(\bmod 2) \text {. }
$$


Sejam $M$ e $N$ variedades $n$-dimensionais orientadas sem bordo, e seja $f: M \rightarrow N$ uma aplicação suave. Se $M$ é compacta e $N$ é conexa, então o grau de $f$ é definido como a seguir:

Seja $x \in M$ um ponto regular de $f$, assim, $d f_{x}: T_{x} M \rightarrow T_{f(x)} N$ é um isomorfismo linear entre dois espaço vetoriais. Defina o sinal de $d f_{x}\left(\right.$ denotaremos por $\left.\operatorname{sign}\left(d f_{x}\right)\right)$ como +1 ou -1 de acordo com $d f_{x}$ preservar ou não a orientação. Para qualquer valor regular $y \in N$, defina

$$
\operatorname{deg}(f ; y)=\sum_{x \in f^{-1}(y)} \operatorname{sign}\left(d f_{x}\right) .
$$

Para os resultados abaixo, vamos considerar $M$ o bordo de uma variedade compacta e orientada $X$, com a orientação induzida por $X$, isto é, escolhemos uma base para os espaço tangente ao bordo e um vetor que aponta para fora, como feito seção 1.2.

Lema 2.5 : Se $f: M \rightarrow N$ pode ser estendida a uma aplicação $F: X \rightarrow N$, então $\operatorname{deg}(f ; y)=0$ para todo valor regular $y \in N$.

Demonstração: Primeiramente, suponha que $y$ seja um valor regular de $F$. A variedade compacta 1 -dimensional $F^{-1}(y)$ é a união finita de círculos e arcos, sendo os pontos do bordo em $M$ e nos arcos. Seja $A \subset F^{-1}(y)$ um destes arcos, com $\partial A=\{a\} \cup\{b\}$. Vamos mostrar que a soma dos sinais de $f$ em $A$ é zero.

As orientações de $X$ e $N$ determinam uma orientação para $A$ da seguinte maneira: Dado $x \in A$, seja $\left(v_{1}, \ldots, v_{n+1}\right)$ uma base positivamente orientada para $T_{x} X$, com $v_{1}$ tangente a $A$. Então $v_{1}$ determina a orientação que queremos para $T_{x} A$ se, e somente se, $d F_{x}$ leva $\left(v_{2}, \ldots, v_{n+1}\right)$ em um base positivamente orientada de $T_{y} N$. Seja $v_{1}(x)$ o vetor unitário positivamente orientado em $x$, tangente a $A$. Temos que $v_{1}$ é uma função suave e aponta para fora em um dos pontos do bordo de $A$, digamos $b$, e aponta para dentro no outro ponto do bordo de $A$, digamos $a$. Disto segue que

$$
\operatorname{sign}\left(d f_{a}\right)=-1 \quad \operatorname{sign}\left(d f_{b}\right)=+1
$$


Portanto, fazendo a soma em todos os arcos, temos que $\operatorname{deg}(f ; y)=0$. Pelo mesmo argumento que usamos anteriormente, se y não é um valor regular de $F$, tomamos uma vizinhança de $y$ de valores regulares de $f$, basta escolher um valor regular de $F$ e teremos o resultado.

Lema 2.6 : Se y é um valor regular de $f, g: M \rightarrow N$, com $f \sim g$ e $M=\partial X$, então $\operatorname{deg}(g ; y)=\operatorname{deg}(f ; y)$

Demonstração: A variedade $[0,1] \times M$ pode ser orientada como um produto, e terá como bordo $1 \times M$, orientado positivamente, e $0 \times M$, orientado negativamente. Assim, o grau de $\left.F\right|_{\partial([0,1] \times M)}$ em um valor regular $y$ é igual a diferença

$$
\operatorname{deg}(g ; y)-\operatorname{deg}(f ; y)
$$

a qual deve ser zero, pelo lema anterior.

Teorema 2.7 : O inteiro deg(f;y) não depende da escolha do valor regular $y$.

Demonstração: Sejam $y$ e $z$ dois valores regulares de $f$. Seja $h: N \rightarrow N$ um difeomorfismo levando $y$ em $z$, isotópico a identidade. Então $h$ preserva orientação e

$$
\operatorname{deg}(f ; y)=\operatorname{deg}(h \circ f ; h(y))=\operatorname{deg}(f ; z) .
$$

De fato,

$$
\begin{aligned}
\operatorname{deg}(h \circ f ; h(y)) & =\sum_{x \in\left((h \circ f)^{-1}(y)\right)} \operatorname{sign}\left(d(h \circ f)_{h(y)}\right)=\sum_{x \in f^{-1}(z)} \operatorname{sign}\left(d h_{f(z)} \cdot d f_{z}\right) \\
& =\sum_{x \in f^{-1}(z)} \operatorname{sign}\left(d f_{z}\right)
\end{aligned}
$$

pois $\operatorname{sign}\left(d h_{f(z)}\right)=1$. Portanto, pelo Lema 2.6, segue o resultado desejado. 
Teorema 2.8 : Se $f$ é suavemente homotópica a $g$, então $\operatorname{deg}(f)=\operatorname{deg}(g)$.

Demonstração: Basta aplicar o Lema 2.6, juntamente com o Teorema anterior, e o resultado estará provado.

Exemplo 2.9 : Considere sobre a esfera a reflexão

$$
r_{i}\left(x_{1}, \ldots, x_{n+1}\right)=\left(x_{1}, \ldots, x_{i-1},-x_{i}, x_{i+1}, \ldots, x_{n+1}\right),
$$

que inverte a orientação. A aplicação antípoda é a composição de $n+1$ reflexões, $r_{1} \circ r_{2} \circ \ldots \circ r_{n+1}$, tendo assim grau $(-1)^{n+1}$.

Note que sobre a esfera $S^{n} \subset \mathbb{R}^{k+1}, v(x)$ ser um campo tangente é equivalente a condição $v(x) \cdot x=0$, para todo $x \in S^{n}$, usando o produto interno canônico. Se v(x)é não nulo para todo $x$, então podemos supor $v(x) \cdot v(x)=1, \forall x \in S^{n}$, bastando tomar $\bar{v}(x)=\frac{v(x)}{\|v(x)\|}$. Sendo assim, podemos ver $v$ como uma aplicação suave de $S^{n}$ nela própria.

Agora, defina a homotopia suave

$$
\begin{aligned}
F: S^{n} \times[0, \pi] & \rightarrow S^{n} \\
(x, \theta) & \mapsto x \cdot \cos (\theta)+v(x) \cdot \operatorname{sen}(\theta) .
\end{aligned}
$$

Após cálculos elementares, obtemos

$$
F(x, \theta) \cdot F(x, \theta)=1, \quad F(x, 0)=x \quad \text { e } \quad F(x, \pi)=-x .
$$

Logo, caso n seja ímpar, a aplicação antípoda é homotópica a identidade. Por outro lado, se n é par isto não acontece, visto que a aplicação antípoda inverte a orientação. 


\section{2 Índices de Campos de Vetores e o Teorema de Poincaré-Hopf}

Definição 2.10 : Um campo de vetores tangentes à superfície $M \subset \mathbb{R}^{n}$ é uma aplicação $v: M \rightarrow \mathbb{R}^{n}$ tal que $v(p) \in T_{p} M, \forall p \in M$. Uma singularidade do campo $v$ é um ponto $p \in M$ tal que $v(p)=0 \in T_{p} M$. Uma singularidade é isolada se existe vizinhança em $M$ tal que esta é a única singularidade nesta vizinhança.

Consideremos agora um aberto $U \subset \mathbb{R}^{m}$ e campo de vetores $v: U \rightarrow \mathbb{R}^{m}$, com um zero isolado em $z \in U$. A função

$$
\bar{v}(x)=\frac{v(x)}{\|v(x)\|}
$$

leva uma pequena esfera centrada em $z$ na esfera unitária. O grau de Brouwer desta aplicação é chamado de índice $\iota$ do campo $v$ no zero $z$.

Exemplo 2.11 : Em $\mathbb{R}^{2}$, ao fazermos um pequeno círculo em torno de z, podemos contar o "número de voltas" (no sentido anti-horário contamos positivamente e no sentido horário negativamente) que o campo tangente a este círculo faz. Este número de voltas será o índice ı do zero $z$, por exemplo, veja as figuras 2.3, 2.4, 2.5 e 2.6.

Vamos provar que esta definição de índice é invariante por difeomorfismos de $U$.

Definição 2.12 : Os campos de vetores $v$ em $M$ e v' em $N$ são equivalentes se existe aplicação $f$ se a derivada $d f_{x}$ leva $v(x)$ em $v^{\prime}(x)$, para cada $x \in M$. Se $f$ é um difeomorfismo, então v' é unicamente determinado por $v$. Em particular,

$$
v^{\prime}=d f \circ v \circ f^{-1}
$$

Lema 2.13 : Suponha que o campo de vetores $v$ em $U$ seja correspondente ao campo $v^{\prime}=d f \circ v \circ f^{-1}$ em $U^{\prime}$, via $f: U \rightarrow U^{\prime}$. Então o índice de $v$ em um zero isolado z é 


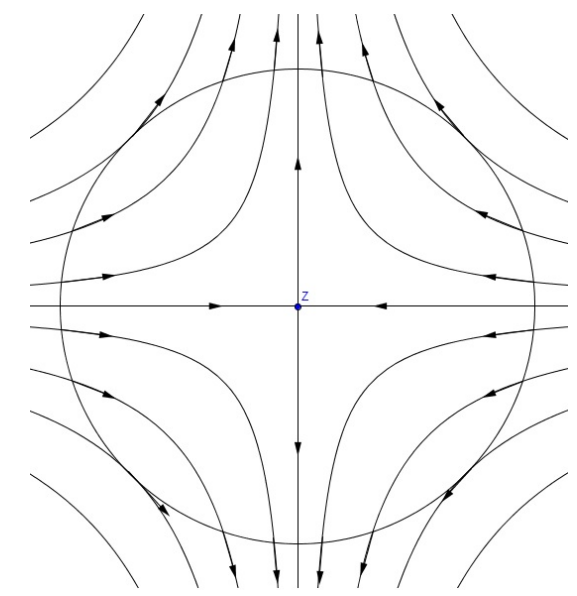

Figura 2.3: Índice -1

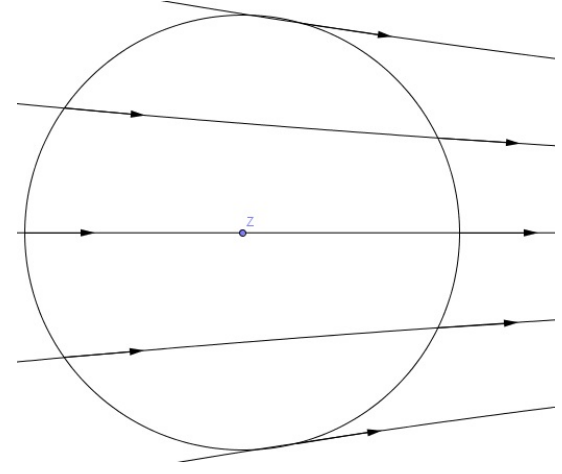

Figura 2.4: Índice 0

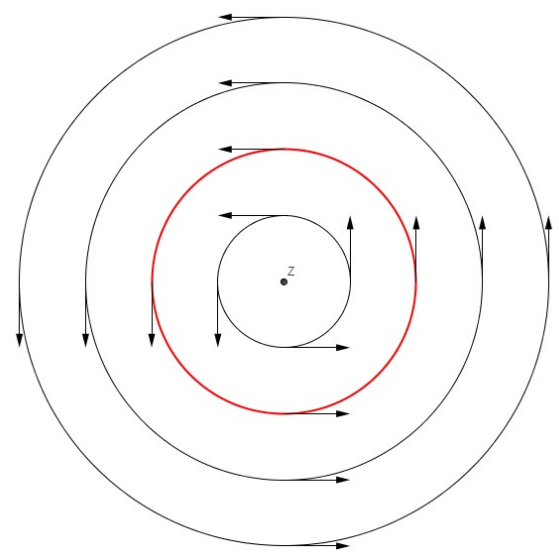

Figura 2.5: Índice 1

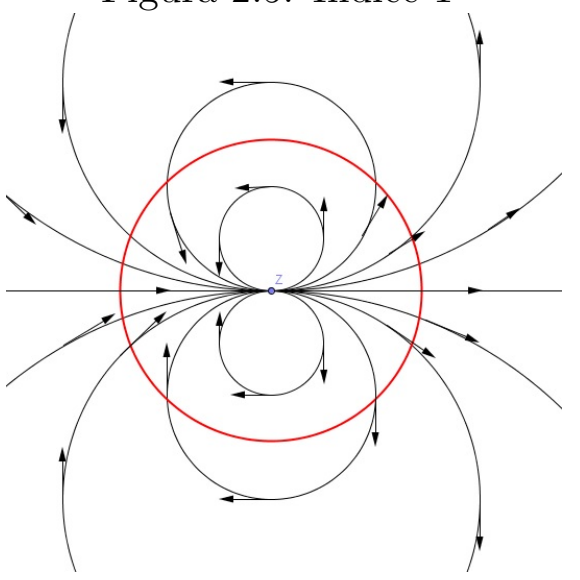

Figura 2.6: Índice 2

igual ao indice de $v^{\prime}$ em $f(z)$.

Demonstração: Podemos assumir que $z=f(z)=0$, pois basta tomarmos translações, que são difeomorfismos. Podemos também tomar $U$ convexo, pois na vizinhança do ponto basta tomar uma pequena bola. Se $f$ preserva orientação, vamos construir uma família a 1-parâmetro de mergulhos $f_{t}: U \rightarrow \mathbb{R}^{m}$, com $f_{0}=i d, f_{1}=f$ e $f_{t}(0)=0$, para todo $t \in[0,1]$. Vamos considerar $v_{t}$ o campo de vetores $d f_{t} \circ v \circ f_{t}^{-1}$ em $f_{t}(U)$, correspondente a $v$ em $U$. Este campo de vetores está bem definido (visto que $d f_{t}$ está definida no espaço tangente a $U$ ) e é não nulo em uma pequena esfera centrada em 0 (pois $z=0$ é um zero isolado de $f$ ). Assim, o índice de $v=v_{0}$ em 0 deve ser igual ao índice de $v^{\prime}=v_{1}$ em 0 , e o lema está provado no caso em que o difeomorfismo preserva 
a orientação.

No caso do difeomorfismo reverter a orientação, basta considerar o caso da reflexão $\rho$. Então

$$
v^{\prime}=\rho \circ v \circ \rho^{-1}
$$

assim, a função associada $\bar{v}^{\prime}(x)=\frac{v^{\prime}(x)}{\left\|v^{\prime}(x)\right\|}$, definida da $\varepsilon$-esfera, satisfaz

$$
\bar{v}^{\prime}=\rho \circ \bar{v} \circ \rho^{-1}
$$

voltando ao caso anterior. Deste modo, temos que o grau de $\bar{v}^{\prime}$ é igual o grau de $\bar{v}$.

Lema 2.14 : Qualquer difeomorfismo de $\mathbb{R}^{m}$ que preserva orientação é suavemente isotópico à identidade.

Demonstração: Podemos novamente assumir que $f(0)=0$. Defina

$$
F: \mathbb{R}^{m} \times[0,1] \rightarrow \mathbb{R}^{m}, \quad \text { por } \quad F(x, t)=\left\{\begin{array}{l}
\frac{f(t x)}{t}, \text { se } 0<t \leq 1 \\
d f_{0}(x), \text { se } t=0
\end{array}\right.
$$

Lema 2.15 : (Lema de Hadamard) Toda função $f$ suave em torno de um ponto z pode ser representada da forma

$$
f(x)=f(z)+\sum_{i=1}^{n}\left(x_{i}-z_{i}\right) g_{i}(x),
$$

com as aplicações $g_{i}$ suaves, $\forall i=1, \ldots, n$.

Note que, pelo Lema de Hadamard (a demonstração pode ser encontrada em [21]), podemos escrever

$$
f(x)=x_{1} g_{1}(x)+\ldots+x_{m} g_{m}(x) .
$$

Deste modo, temos

$$
F(x, t)=x_{1} g_{1}(t x)+\ldots+x_{m} g_{m}(t x) .
$$


Então, $f$ é isotópica à aplicação linear $d f_{0}$, que é isotópica à identidade.

Definição 2.16 : Seja $X \subset \mathbb{R}^{m}$ uma $m$-variedade compacta com bordo. A aplicação de Gauss

$$
g: \partial X \rightarrow S^{m-1}
$$

associa a cada ponto $x \in \partial X$, um vetor unitário normal que aponta para fora da variedade em $x$.

Lema 2.17: (Hopf) Se $v: X \rightarrow \mathbb{R}^{m}$ é um campo de vetores suaves com zeros isolados, e se $v$ aponta para fora de $X$ ao longo do bordo, então a soma dos índices sobre as singularidades do campo $\sum \iota$ é igual ao grau da aplicação de Gauss de $\partial X$ em $S^{m-1}$. Em particular, ı não depende da escolha de $v$.

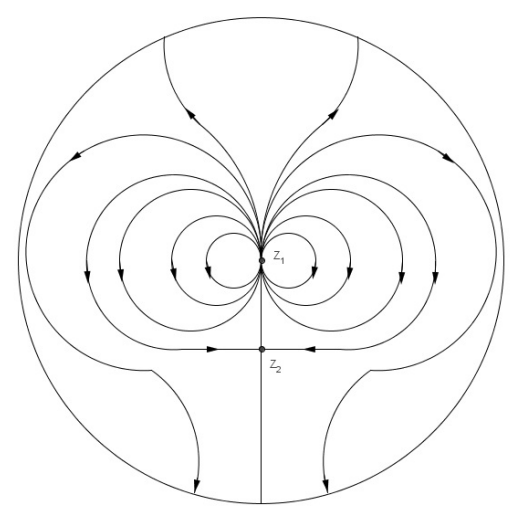

Figura 2.7: Índices 2 em $z_{1}$ e -1 em $z_{2}$, soma dos índices igual a 1

Demonstração: Removendo uma $\varepsilon$-bola de cada zero, obtemos uma nova variedade com bordo (note que o número de singularidades é finito, visto que a variedade é compacta). A aplicação $\bar{v}=\frac{v(x)}{\|v(x)\|}$ leva esta variedade na esfera $S^{m-1}$. Então, a soma dos graus de $\bar{v}$ é igual a zero. Temos que $\left.\bar{v}\right|_{\partial X}$ é homotópico a $g$, ver [15], pg. 507. Além disso, temos que os graus nas outras componentes do bordo contribuem com valor negativo, pois têm a orientação oposta, no sentido que um vetor que chega na 
singularidade, está saindo naquele bordo e vice-versa. Deste modo, temos

$$
\operatorname{deg}(g)-\sum \iota=0
$$

Definição 2.18 : O campo de vetores $v$ é não degenerado em um zero z se a transformação linear $d v_{z}: \mathbb{R}^{m} \rightarrow \mathbb{R}^{m}$ é não singular.

Neste caso, usando o teorema da função inversa, concluímos que $z$ é um zero isolado.

Lema 2.19: O indice de $v$ em um zero não degenerado é igual $a+1$, se $\operatorname{det}\left(d v_{z}\right)>0$ $e-1$, se $\operatorname{det}\left(d v_{z}\right)<0$.

Demonstração: Podemos ver o campo $v$ como um difeomorfismo de alguma vizinhança convexa $U_{0}$ de $z$ em $\mathbb{R}^{m}$. Podemos também assumir, sem perda de generalidade, que $z=0$. Se $v$ preserva a orientação, podemos deformar $\left.v\right|_{U_{0}}$ na identidade sem novos zeros, utilizando os Lemas 2.13 e 2.14. Então o índice certamente é igual a 1. Por outro lado, se $v$ inverte a orientação, temos que $\left.v\right|_{U_{0}}$ pode ser deformado em uma reflexão, donde $\iota=-1$.

Mais geralmente, considere um zero $z$ de um campo vetorial $w$ em uma variedade $M \subset \mathbb{R}^{k}$. Considerando $w$ como uma aplicação de $M$ em $\mathbb{R}^{k}$, a derivada $d w_{z}: T M_{z} \rightarrow$ $\mathbb{R}^{k}$ está bem definida.

Lema 2.20 : A derivada dw leva $T M_{z}$ no subespaço $T M_{z} \subset \mathbb{R}^{k}$, e pode ser considerada como transformação linear de $T M_{z}$ nele próprio. Se esta transformação linear tem determinante $D \neq 0$ então z é um zero isolado de $w$ com índice +1 ou -1 respectivamente quando D é positivo ou negativo.

Demonstração: Seja $h: U \rightarrow M$ uma parametrização de alguma vizinhança de $z$. Seja $e^{i}$ o $i$-ésimo vetor da base de $z R^{m}$, e seja

$$
t^{i}=d h_{u}\left(e^{i}\right)=\frac{\partial h}{\partial u_{i}},
$$


então os vetores $t^{1}, \ldots, t^{m}$ formam uma base para o espaço tangente $T M_{h(u)}$. Devemos calcular a imagem de $t^{i}=t^{i}(u)$, sobre a transformação linear $d w_{h(u)}$. Primeiramente, note que

$$
d w_{h(u)}\left(t^{i}\right)=d(w \circ h)_{u}\left(e^{i}\right)=\frac{\partial w(h(u))}{\partial u_{i}} .
$$

Seja $v=\sum v_{i} e^{i}$ o vetor em $U$ que corresponde ao campo $w$ em $M$. Por definição, $v=d h^{-1} \circ w \circ h$, tal que

$$
w(h(u))=d h_{u}(v) \sum v_{i} t^{i}
$$

Assim,

$$
\frac{\partial w(h(u))}{\partial u_{i}}=\sum_{i}\left(\frac{\partial v_{i}}{\partial u_{i}}\right) t^{i}+\sum_{i} v_{i}\left(\frac{\partial t^{i}}{\partial u_{i}}\right) .
$$

Por (1), (2) e calculando no zero $h^{-1}(z)$ de $v$, nós obtemos a fórmula

$$
d w_{z}\left(t^{i}\right)=\sum_{i}\left(\frac{\partial v_{i}}{\partial u_{i}}\right) t^{i}
$$

Então $d w_{z}$ leva $T M_{z}$ nele mesmo e o determinante $D$ desta transformação linear $T M_{z} \rightarrow T M_{z}$ é igual ao determinante da matriz $\left(\frac{\partial v_{i}}{\partial u_{i}}\right)$. Aplicando o lema anterior, a prova está completa.

Considere $M \subset \mathbb{R}^{k}$ uma variedade suave, compacta sem bordo. Seja $N_{\varepsilon}$ a $\varepsilon$-vizinhança fechada de $M$, isto é

$$
N_{\varepsilon}=\left\{x \in \mathbb{R}^{k} ;\|x-y\| \leq \varepsilon, \text { para algum } y \in M\right\}
$$

Teorema 2.21 : Para qualquer campo de vetores $v$ em $M$ com apenas zeros não degenerados. A soma dos índices $\sum \iota$ é igual ao grau da aplicação de Gauss

$$
g: \partial N_{\varepsilon} \rightarrow S^{k-1}
$$

Em particular, esta soma não depende da escolha do campo $v$. 


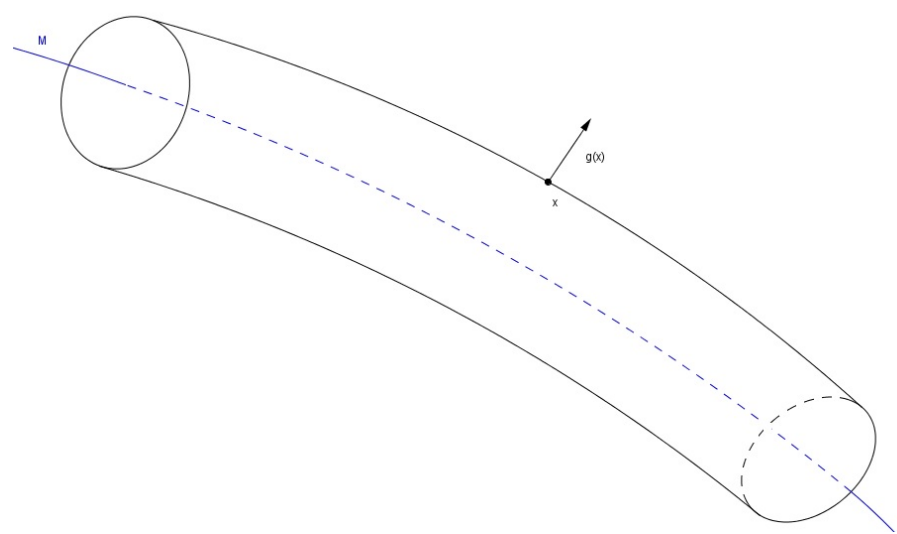

Figura 2.8: $\varepsilon$ vizinhança tubular

Demonstração: Para $x \in N_{\varepsilon}$, seja $r(x) \in M$ o ponto mais próximo de $x$. Note que o vetor $x-r(x)$ é perpendicular ao espaço tangente de $M$ em $r(x)$, pois do contrário $r(x)$ não será o ponto mais próximo em $M$. Se $\varepsilon$ é suficientemente pequeno, a função $r(x)$ está bem definida e é suave. Considere a função distância ao quadrado

$$
\phi(x)=\|x-r(x)\|^{2} .
$$

Agora, note que

$$
\nabla \phi(x)=2(x-r(x))+2 r^{\prime}(x) \cdot(x-r(x)) .
$$

Logo, como $r^{\prime}(x)$ é perpendicular a $x-r(x)$, temos que $\nabla \phi(x)=2(x-r(x))$. Assim, para cada ponto $x$ da superfície de nível $\partial N_{\varepsilon}=\phi^{-1}\left(\varepsilon^{2}\right)$, o vetor unitário que aponta para fora é dado por

$$
g(x)=\frac{\nabla \phi}{\|\nabla \phi\|}=\frac{x-r(x)}{\varepsilon} .
$$

Estenda $v$ a um campo $w$ na vizinhança de $N_{\varepsilon}$ fazendo

$$
w(x)=(x-r(x))+v(r(x)) .
$$

Temos que $w$ aponta para fora nos pontos do bordo, visto que o produto interno 
$w(x) \cdot g(x)$ é igual a $\varepsilon>0$. Note que $w$ pode se anular apenas nos zeros de $v$ em $M$, e isto decorre do fato que $x-r(x)$ e $v(r(x))$ são ortogonais entre si. Computando a derivada de $w$ em um zero $z \in M$, temos que

$$
\left\{\begin{array}{lll}
d w_{z}(h)=d v_{z}(h) & \text { se } & h \in T M_{z} \\
d w_{z}(h)=h & \text { se } & h \in T M_{z}^{\perp}
\end{array}\right.
$$

Então, o determinante de $d w_{z}$ é igual ao determinante de $d v_{z}$. Assim, o índice de $w$ em um zero $z$ é igual ao índice $\iota$ de $v$ em $z$. Portanto, pelo Lema 2.17, temos que o somatório dos índices $\sum \iota$ é igual ao grau de $g$, o que prova o teorema.

Teorema 2.22 : (Poincaré-Hopf) A soma $\sum \iota$ de indices nos zeros de um campo de vetores é igual ao número de Euler

$$
\chi(M)=\sum_{i=0}^{m}(-1)^{i} \operatorname{rank} H_{i}(M) .
$$

Onde $H_{i}(M)$ é o i-ésimo grupo de homologia de $M$. Em particular esta soma é um invariante topológico de $M$.

Note que o Lema 2.17 e o teorema anterior são versões particulares do teorema de Poincaré-Hopf. Não faremos a demonstração precisa do teorema, mas daremos uma ideia da demonstração a seguir.

Passo 1: Identificar o invariante $\sum \iota$ com o número de Euler $\chi(M)$. Para isto é suficiente construir um exemplo de um campo de vetores não degenerado em $M$ com $\sum \iota$ igual a $\chi(M)$. Marston Morse mostrou que é possível construir uma função a valores reais em $M$ cujo gradiente é um campo de vetores não degenerado. Além disso, Morse também mostrou que a soma dos índices deste campo é igual ao número de Euler de $M$.

Passo 2: Provar o teorema para um campo de vetores com zeros degenerados. Considere um campo de vetores $v$ em um aberto $U$ com um zero isolado em $z \in U$. 
Se a função $\lambda: U \rightarrow[0,1]$ vale 1 em uma vizinhança $N$ de $z$ e vale 0 fora de uma vizinhança um pouco maior $N$ de $z$, e se $y$ é um valor regular de $v$ suficientemente pequeno, então o campo

$$
v^{\prime}(x)=v(x)-\lambda(x) \cdot y
$$

é não degenerado em $N$. Note que $\lambda$ existe pelo Lema de Urysohn. A soma dos índices nos zeros dentro de $N$ podem ser calculados como o grau da aplicação

$$
\bar{v}: \partial N \rightarrow S^{m-1}
$$

logo não muda fazendo esta alteração. Mais geralmente, o argumento em uma variedade compacta $M$ é trocar um campo de vetores degenerado por um que não seja degenerado, sem trocar o inteiro $\sum \iota$.

Passo 3: Variedades com bordo. Se $M \subset \mathbb{R}^{k}$ tem um bordo,, então qualquer campo $v$ que aponta para fora nos pontos do bordo $\partial M$ pode ser estendido, como anteriormente, a um campo sobre $N_{\varepsilon}$ que também aponta para fora em $\partial N_{\varepsilon}$. Entretanto, dessa forma não é possível manter a suavidade no bordo de $M$. Além disso, temos que $N_{\varepsilon}$ como na figura do Teorema 2.21, não pode ser uma variedade suave $\left(C^{\infty}\right)$, somente $C^{1}$. Deste modo, a extensão $w$ definida por $w(x)=v(r(x))+x-r(x)$ será apenas um campo contínuo próximo de $\partial M$. Para continuar a demonstração seria necessário mostrar que nossas suposições sobre a diferenciabilidade podem ser removidas.

Teorema 2.23 : (Poincaré-Hopf) Se um campo contínuo de vetores tangentes a uma superfície compacta $M$ tem apenas um número finito de singularidades, então a soma dos índices dessas singularidades é igual à característica de Euler-Poincaré de $M$.

O Teorema 2.23 nos diz que se $v$ é um campo contínuo de vetores tangentes a uma superfície compacta $M$ e o conjunto de singularidades $S$ é finito, então

$$
\sum_{p \in S} i(v, p)=\chi(M)
$$


Temos o seguinte corolário do Teorema de Poincaré-Hopf

Corolário 2.24 : Se $\chi(M) \neq 0$ então todo campo contínuo de vetores tangentes se anula em algum ponto.

A esfera $S^{2}$, por exemplo, não possui campo de vetores tangentes contínuo sem pontos críticos.

No toro $T^{2}$ temos que $\chi\left(T^{2}\right)=0$. Podemos definir o campo de vetores tangentes aos paralelos do toro.

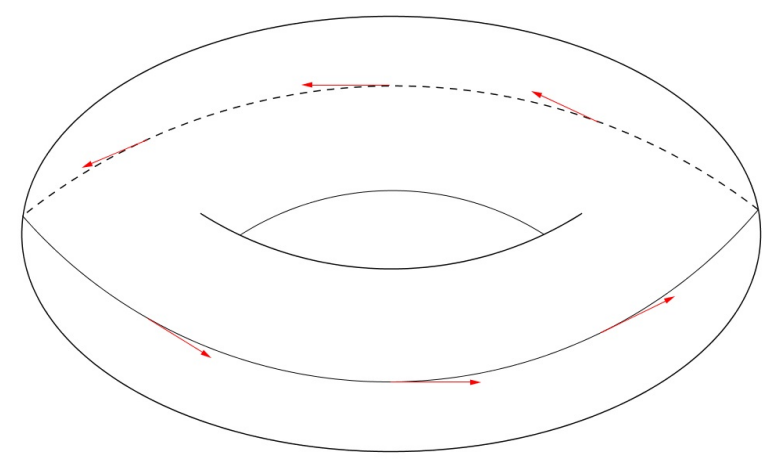

Figura 2.9: Campo sobre $T^{2}$

Seja $n$ ímpar (suponha $n=2 k-1$ ), então a esfera unitária $S^{n}$ é o conjunto

$$
S^{n}=\left\{p=\left(x_{1}, y_{1}, x_{2}, y_{2}, \ldots, x_{k}, y_{k}\right) \in \mathbb{R}^{n+1} ; \sum_{i=1}^{k}\left(x_{i}^{2}+y_{i}^{2}\right)=1\right\}
$$

Um vetor $v$ é tangente à $S^{n}$ no ponto $p$ se $\langle v, p\rangle=0$. Defina em $S^{n}$ o campo $v(p)=$ $\left(y_{1},-x_{1}, y_{2},-x_{2}, \ldots, y_{k},-x_{k}\right)$, que é contínuo e não nulo, uma vez que $v(p)=0 \leftrightarrow p=0$.

O Teorema de Dualidade de Poincaré nos diz que, em uma superfície compacta, os números de Betti módulo 2 equidistantes dos extremos são iguais, isto é, $\beta_{i}=\beta_{m-i}$, onde $\operatorname{dim} M=m$. Assim, temos que

$$
\chi(M)=\sum_{i=1}^{m}(-1)^{i} \cdot \beta_{i}=0 .
$$


A igualdade acima sugere que toda superfície compacta de dimensão ímpar possui campo contínuo de vetores tangentes não nulo. Para superfícies compactas de dimensão 2 contidas em $\mathbb{R}^{3}$ existe um teorema de classificação completo.

Teorema 2.25 : Uma superfície $M^{2} \subset \mathbb{R}^{3}$ compacta é homeomorfa a uma esfera ou a um n-toro, onde $n$ é o gênero (número de buracos) de $M^{2}$. Além disso, se o gênero de $M^{2}$ é g, temos que

$$
\chi\left(M^{2}\right)=2-2 g .
$$

O teorema acima nos diz que apenas o toro possui campo contínuo de vetores tangentes não nulo. 


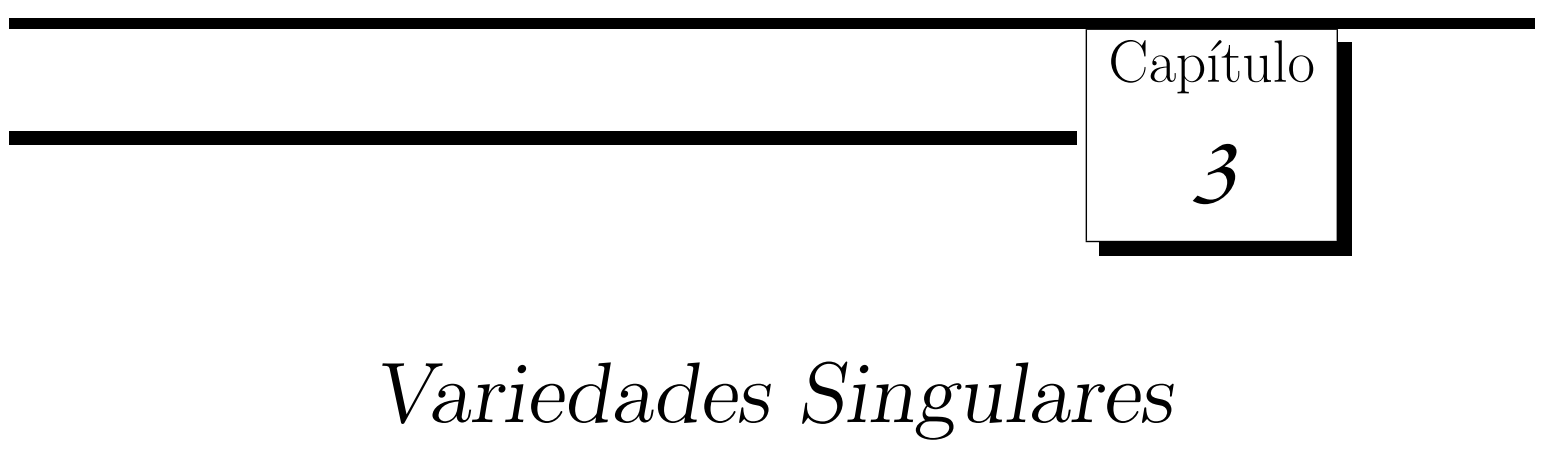

Veremos neste capítulo definições e resultados básicos de conjuntos algébricos e conjuntos analíticos. A principal referência é o livro [20].

\subsection{Conjuntos Algébricos}

Sejam $\mathbb{K}$ um corpo infinito e $\mathbb{K}\left[z_{1}, \ldots, z_{n}\right]$ o anel de polinômios nas variáveis $z_{1}, \ldots, z_{n}$ com coeficientes em $\mathbb{K}$.

Definição 3.1 : Um subconjunto $V \subset \mathbb{K}^{n}$ é um conjunto algébrico se $V$ é o conjunto de zeros de alguma coleção de funções polinomiais em $\mathbb{K}^{n}$.

Denotaremos o conjunto de zeros do ideal $J$ por $Z(J)$. Seja

$$
I(V) \subset \mathbb{K}\left[z_{1}, \ldots, z_{n}\right]
$$

o ideal dos polinômios que se anulam em $V$. O teorema da base de Hilbert garante que todo ideal é gerado (como $\mathbb{K}\left[z_{1}, \ldots, z_{n}\right]$-módulo) por alguma coleção finita de polinômios. Disto segue que todo conjunto algébrico $V$ pode ser definido por alguma coleção finita de equações polinomiais. Seja $S$ um conjunto finito de polinômios. O conjunto $V(S)$ é o conjunto dos pontos $z \in K^{n}$ tais que $f(z)=0, \forall f \in S$.

Definição 3.2 : Um ideal $P$ de um anel $R$ é um ideal primo se $P \neq R$ e se dados $a, b \in R$ tais que $a \cdot b \in P$, então $a \in P$ ou $b \in P$. 
Definição 3.3 : O radical de um ideal I em um anel $R$ é o ideal das intersecções de todos os ideais primos que contém I. Denotaremos o radical de I por $\sqrt{I}$.

Proposição 3.4 Sejam $V_{1}$ e $V_{2}$ conjuntos algébricos e $I_{1}=I\left(V_{1}\right)$ e $I_{2}=I\left(V_{2}\right)$. Então valem as seguintes propriedades:

i. Se $I\left(V_{1}\right) \subset I\left(V_{2}\right)$, então $V_{2} \subset V_{1}$.

ii. $V\left(I_{1} \cap I_{2}\right)=V\left(I_{1} \cdot I_{2}\right)=V\left(I_{1}\right) \cup V\left(I_{2}\right)$.

iii. $V\left(I_{1}+I_{2}\right)=V\left(I_{1}\right) \cap V\left(I_{2}\right)$.

$O$ item $i$. decorre diretamente da definição, de fato, se $z=\left(z_{1}, \ldots, z_{n}\right) \in V_{2}$ e $I\left(V_{1}\right) \subset$ $I\left(V_{2}\right)$ então $f(z)=0, \forall f \in I\left(V_{2}\right)$. Logo, por hipótese, temos $f(z)=0, \forall f \in I\left(V_{1}\right)$, portanto $z \in V_{1}$. A recíproca também é verdadeira e a demonstração é análoga. As demonstrações para ii. e iii. podem ser encontradas em [11], pg. 113.

Teorema 3.5 : (Hilbert-Nullstellensatz) Sejam $\mathbb{K}$ um corpo algebricamente fechado, I um ideal de $R=\mathbb{K}\left[z_{1}, \ldots, z_{n}\right]$ e $f \in R$ um polinômio que se anula em $Z(I)$. Então $f^{r} \in I$ para algum inteiro $r>0$.

Uma formulação equivalente deste teorema é a seguinte:

Teorema 3.6 : (Hilbert-Nullstellensatz) Seja $\mathbb{K}$ um corpo algebricamente fechado. Então, para todo ideal $I$ de $\mathbb{K}\left[z_{1}, \ldots, z_{n}\right]$, temos que $I(Z(I))=\sqrt{I}$.

Uma referência para os teoremas acima é [12].

Proposição 3.7 : Toda sequência decrescente $V_{1} \supset V_{2} \supset V_{3} \supset \ldots$ de conjuntos algébricos termina ou estabiliza $\left(V_{i}=V_{i+1}=V_{i+2} \ldots\right)$ após um número finito de passos.

Demonstração: $\quad$ Segue da Proposição 3.4 olhando para a sequência ascendente $I\left(V_{1}\right) \subset I\left(V_{2}\right) \subset \ldots$ de ideais em $\mathbb{K}\left[z_{1}, \ldots, z_{n}\right]$. Como $\mathbb{K}\left[z_{1}, \ldots, z_{n}\right]$ é Noetheriano (ver [12], pg. 186), segue que esta última sequência deve estabilizar. Portanto, pela Proposição $3.4(i)$, segue que $V_{1} \supset V_{2} \supset \ldots$ deve estabilizar. 
Definição 3.8 : Um conjunto algébrico não vazio $V$ é uma variedade ou um conjunto algébrico irredutivel se não pode ser expresso como união de dois subconjuntos algébricos próprios.

Proposição 3.9 : Um conjunto algébrico $V$ é irredutivel se, e somente se, $I(V)$ é um ideal primo.

Demonstração: Ver [20].

Se $V$ é irredutível, então o corpo dos quocientes $f / g$ com $f$ e $g$ no domínio de integridade $\frac{\mathbb{K}\left[z_{1}, \ldots, z_{m}\right]}{I(V)}$ é chamado corpo de funções racionais em $V$. O grau de transcendência sobre $\mathbb{K}$ é a dimensão algébrica de $V$ sobre $\mathbb{K}$ (ver [13], pg. 28).

Observação 3.10 : Se $W$ é uma subvariedade própria de $V$, então a dimensão de $W$ é menor que a dimensão de $V$ (ver [13], pg. 29).

Seja $V \subset \mathbb{K}^{m}$ um conjunto algébrico não vazio. Escolha $\left\{f_{1}, \ldots, f_{k}\right\}$ polinômios que geram o ideal $I(V)$ e, para cada $z \in V$, considere a matriz $\left(\partial f / \partial z_{j}\right)$ calculada em $z$. Seja $\rho$ o maior posto que esta matriz atinge em qualquer ponto de $V$.

Definição 3.11 : Um ponto $z \in V$ é não singular ou simples se $\left(\partial f / \partial z_{j}\right)$ atinge o posto máximo $\rho$ em z, e é singular se

$$
\operatorname{rank}\left(\partial f_{i}(z) / \partial z_{j}\right)<\rho
$$

Note que esta definição não depende da escolha dos polinômios $f_{i}$, pois se adicionarmos um novo polinômio $f_{k+1}$, temos que este pode ser escrito como combinação linear dos demais, adicionando uma coluna que não interfere no posto da matriz.

Lema 3.12 : O conjunto $\Sigma(V)$ de todos os pontos singulares de $V$ forma um subconjunto algébrico próprio (possivelmente vazio) de $V$.

Demonstração: Basta notar que $z \in \Sigma(V)$ se, e somente se, todo determinante das submatrizes $\rho \times \rho$ se anulam, logo $\Sigma(V)$ é um conjunto de zeros de polinômios. 
Teorema 3.13 (Whitney): Se $\mathbb{K}$ é o corpo $\mathbb{R}$ ou $\mathbb{C}$, então o conjunto $V-\Sigma(V)$ de pontos não singulares de $V$ forma uma variedade suave e não vazia. Além disso, esta variedade é analítica real (ou complexa, dependendo do corpo), e tem dimensão $n-\rho$ sobre $\mathbb{K}$.

Uma referência para a demonstração do resultado acima é [23].

Teorema 3.14 (Whitney): Para qualquer par $V \supset W$ de conjuntos algébricos em um espaço de coordenadas reais ou complexas, a diferença $V-W$ tem no máximo um numero finito de componentes topológicas conexas.

Uma referência para a demonstração do resultado acima é [19], Appendix A.

Note que o teorema anterior nos diz que, se $V$ é uma variedade analítica com um número finito de componentes irredutíveis, então $V-\Sigma(V)$ é uma variedade suave que tem um número finito de componentes conexas.

Exemplo 3.15 : Considere a seguinte variedade em $\mathbb{R}^{2}$

$$
V=\left\{(x, y) \in \mathbb{R}^{2} ; y^{2}-x^{2}\left(1-x^{2}\right)=0\right\}
$$

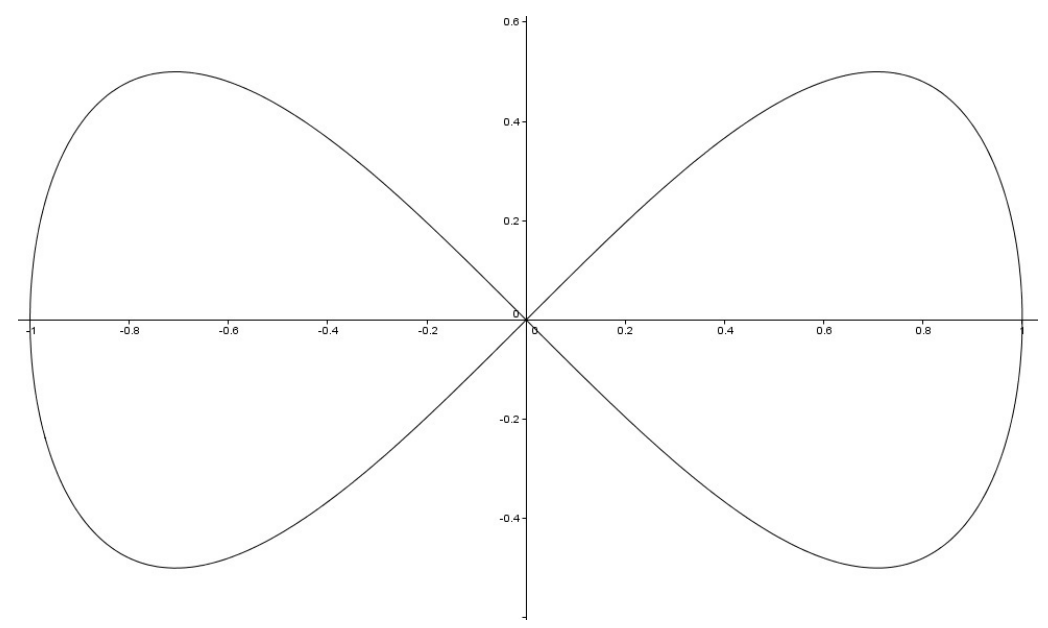

Figura 3.1: Curva $y^{2}-x^{2}\left(1-x^{2}\right)=0$

Esta variedade ilustra um tipo de singularidade chamado ponto duplo e $V-\{0\}$ tem duas componentes reais analíticas. 
Exemplo 3.16 : A curva cúbica

$$
V=\left\{(x, y) \in \mathbb{R}^{2} ; y^{2}-x^{2}(x-1)=0\right\}
$$

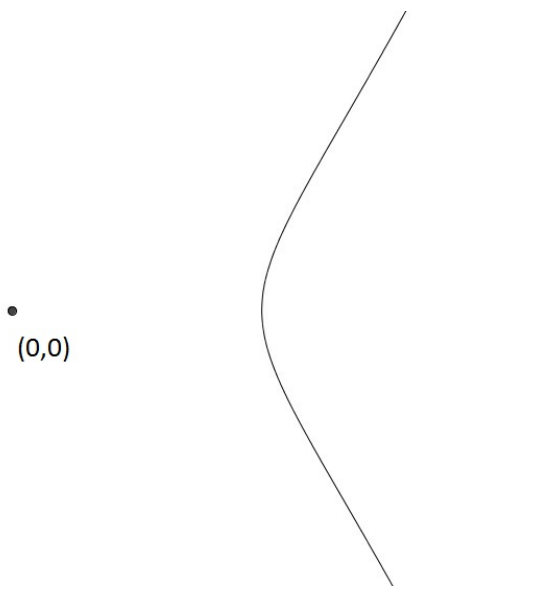

Figura 3.2: Curva $y^{2}-x^{2}(x-1)=0$

tem um zero isolado na origem, esta curva é também irredutível sobre $\mathbb{C}$.

Proposição 3.17 : Uma variedade algébrica complexa nunca pode ser uma variedade suave em toda uma vizinhança de um ponto singular.

Demonstração: Suponha que a variedade complexa $V$ seja uma variedade diferenciável de classe $C^{1}$ em toda uma vizinhança $U$ da origem em $\mathbb{C}^{n}$. O espaço tangente à variedade suave $U \cap V$ em qualquer ponto simples é claramente um espaço vetorial sobre $\mathbb{C}$. Do fato de que os pontos simples formam um conjunto denso (ver [19]) temos que, por continuidade, o espaço tangente (real) $T_{z} \subset \mathbb{C}^{n}$ de $U \cap V$ em um ponto arbitrário $z$ é, na verdade, um espaço vetorial complexo, isto é, $T_{z}=i \cdot T_{z}$. Substituindo $U$ por um aberto menor $U^{\prime}$ e re-enumerando as coordenadas se necessário, o Teorema 1.15 nos garante que $U^{\prime} \cap V$ em um ponto arbitrário $z$ pode ser considerado como gráfico de uma aplicação $C^{1}$-suave $F$ de um aberto do espaço de coordenadas $\left(z_{1}, \ldots, z_{m}\right)$ no espaço de $\left(z_{m+1}, \ldots, z_{n}\right)$ coordenadas. A derivada de $F$ em cada ponto é uma aplicação 
linear complexa, então as equações de Cauchy-Riemann são satisfeitas, e $F$ é complexa analítica. Isto prova que $U^{\prime} \cap V$ é uma variedade complexa suave. Agora, seja $h(z)$ qualquer função complexa analítica, definida em uma vizinhança do 0, que se anula em $V$, e sejam $f_{1}, \ldots, f_{k}$ polinômios que geram o ideal primo $I(V) \subset \mathbb{C}\left[z_{1}, \ldots, z_{n}\right]$. O Teorema 3.5 (ne verdade a versão local analítica deste teorema, que pode ser encontrada em [8], pg.97) garante que existe $s_{0} \in \mathbb{N}$ tal que para todo $s \geq s_{0}$ potências $h^{s}$ podem ser expressas como uma combinação linear da forma $a_{1} f_{1}+\ldots+a_{k} f_{k}$, onde $a_{1}, \ldots, a_{k}$ são germes de funções analíticas. Passando para o maior anel $\mathbb{C}[[z]]$ que consiste de todas as séries formais de potências na origem, segue que $h^{s} \in \mathbb{C}[[z]] I(V)$. Mas este ideal pode ser expresso como a intersecção de ideais primos. Logo o próprio $h$ deve pertencer ao ideal $\mathbb{C}[[z]] I(V)$, que é gerado por $f_{1}, \ldots, f_{k}$ em $\mathbb{C}[[z]]$. Olhando para as derivadas, temos que o vetor $d h(0)$ pode ser escrito como combinação linear complexa de $d f_{1}(0), \ldots, d f_{k}(0)$. Disto segue que a matriz $\left(\frac{\partial f_{i}}{\partial z_{j}}\right)$ tem posto $n-m$ em 0 , portanto a origem não pode ser um ponto singular de $V$.

Teorema 3.18 : Qualquer conjunto algébrico $V$, real ou complexo, pode ser escrito como uma união finita e disjunta

$$
V=M_{1} \cup M_{2} \cup \ldots \cup M_{p}
$$

onde cada $M_{j}$ é uma variedade suave com um número finito de componentes.

Demonstração: $\quad$ Pelo Lema 3.12 e pelo Teorema de Whitney 3.13, sabemos que $\Sigma(V)$ é um conjunto algébrico e que $V-\Sigma(V)$ é uma variedade suave. Sejam $M_{1}=$ $V-\Sigma(V), M_{2}=\Sigma(V)-\Sigma(\Sigma(V))$, e assim por diante. Temos em $V$ a condição da cadeia descendente, isto é, sequência

$$
V \supset V-\Sigma(V) \supset \Sigma(V)-\Sigma(\Sigma(V)) \supset \ldots
$$


deve terminar. Portanto,

$$
V=M_{1} \cup \ldots \cup M_{p}, \quad \text { para algum } p \text {. }
$$

Além disso, se $W \subset V$, podemos expressar $V-W=M_{1}^{\prime} \cup \ldots \cup M_{p}^{\prime}$ onde cada

$$
M_{i}^{\prime}=M_{i}-\left(W \cap M_{i}\right)
$$

é uma variedade suave com número finito de componentes topológicas de acordo com o Teorema de Whitney 3.14 .

Lema 3.19 : Sejam $\mathbb{K}$ o corpo dos reais ou complexos, $V \subset \mathbb{K}^{m}$ um conjunto algébrico, $M_{1}=V-\Sigma(V)$ a variedade dos pontos simples de $V$ e $g$ uma função polinomial em $\mathbb{K}^{m}$. Então, o conjunto de pontos críticos de $\left.g\right|_{M_{1}}: M_{1} \rightarrow \mathbb{K}$ é igual a intersecção de $M_{1}$ com o conjunto algébrico $W$ que consiste de todos os pontos $z \in V$ nos quais a matriz

$$
\left(\begin{array}{ccc}
\frac{\partial g}{\partial z_{1}} & \cdots & \frac{\partial g}{\partial z_{n}} \\
\frac{\partial f_{1}}{\partial z_{1}} & \cdots & \frac{\partial f_{1}}{\partial z_{n}} \\
\vdots & & \vdots \\
\frac{\partial f_{k}}{\partial z_{1}} & \cdots & \frac{\partial f_{k}}{\partial z_{n}}
\end{array}\right)
$$

tem posto menor ou igual a $\rho=\min \{k, n\}$, onde $f_{1}, \ldots, f_{k}$ denotam os polinômios que $\operatorname{geram} I(V)$.

Demonstração: Nas proximidades de qualquer ponto de $M_{1}$ podemos escolher um sistema analítico (real ou complexo) de vizinhanças coordenadas $u_{1}, \ldots, u_{n}$ para $\mathbb{K}^{n}$ tal que $M_{1}$ corresponde localmente a $u_{1}=\ldots=u_{\rho}=0$. Então $u_{\rho+1}, \ldots, u_{n}$ podem ser tomadas como coordenadas locais de $M_{1}$. Note que $\frac{\partial f_{i}}{\partial u_{j}}$, calculado em um ponto de $M_{1}$, é zero para $j>\rho+1$ (Teorema de Whitney 3.13). Como a matriz $N=\left(\frac{\partial f_{i}}{\partial u_{j}}\right)$ é equivalente por colunas a matriz $\left(\frac{\partial f_{i}}{\partial z_{l}}\right)$, temos que $W$ tem posto $\rho$. Isto mostra que as primeiras $\rho$ colunas são linearmente independentes. Assim, aumentando a matriz $N$ 
com as derivadas parciais da função $g$, temos que a matriz

$$
\left(\begin{array}{ccc}
\frac{\partial g}{\partial u_{1}} & \cdots & \frac{\partial g}{\partial u_{n}} \\
\frac{\partial f_{1}}{\partial u_{1}} & \cdots & \frac{\partial f_{1}}{\partial u_{n}} \\
\vdots & & \vdots \\
\frac{\partial f_{k}}{\partial u_{1}} & \cdots & \frac{\partial f_{k}}{\partial u_{n}}
\end{array}\right)
$$

deve ter posto $\rho$ se, e somente se,

$$
\frac{\partial g}{\partial u_{\rho+1}}=\ldots=\frac{\partial g}{\partial u_{n}}=0
$$

ou, em outras palavras, se o ponto dado é um ponto crítico de $\left.g\right|_{M_{1}}$. Como esta matriz aumentada é equivalente a matriz $(*)$, temos o desejado.

Corolário 3.20 : Uma função polinomial g em $M_{1}=V-\Sigma(V)$ pode ter no máximo um número finito de valores críticos.

Demonstração: $\mathrm{O}$ conjunto de pontos críticos de $\left.g\right|_{M_{1}}$ pode ser expresso como a diferença $W-\Sigma(V)$ de conjuntos algébricos, que por sua vez pode ser expressa como união finita de variedades suaves da forma

$$
W-\Sigma(V)=M_{1}^{\prime} \cup \ldots \cup M_{p}^{\prime}
$$

onde cada $M_{i}^{\prime}$ tem apenas um número finito de componentes. Cada ponto $x \in M_{i}^{\prime}$ é um ponto crítico da função suave $\left.g\right|_{M_{1}}$, consequentemente, é um ponto crítico da função restrição $\left.g\right|_{M_{i}^{\prime}}$. Como todos os pontos de $M_{i}^{\prime}$ são críticos, temos que $g$ é constante em cada componente de $M_{i}^{\prime}$, isso decorre de uma função contínua levar conexo em conexo. Logo, obtemos que a imagem $g\left(M_{i}^{\prime}\right)$ é um conjunto finito. Mas a união $g\left(M_{1}^{\prime}\right) \cup \ldots \cup g\left(M_{p}^{\prime}\right)$ é exatamente o conjunto de todos os valores críticos de $\left.g\right|_{M_{1}}$.

Consideremos $V$ um conjunto algébrico real ou complexo. Seja $x_{0}$ um ponto simples de $V$ ou um ponto singular isolado do conjunto $\Sigma(V)$. 
Corolário 3.21 : Toda esfera suficientemente pequena $S_{\varepsilon}$ centrada em $x_{0}$ intersecta $V$ em uma variedade suave, possivelmente vazia.

Demonstração: No caso real, vamos aplicar o corolário anterior à função polinomial

$$
r(x)=\left\|x-x_{0}\right\|^{2} .
$$

Se $\varepsilon^{2}$ for menor que qualquer valor crítico de $\left.r\right|_{(V-\Sigma(V))}$, então $\varepsilon^{2}$ será um valor regular. Portanto sua imagem inversa

$$
r^{-1}\left(\varepsilon^{2}\right) \cap(V-\Sigma(V))=S_{\varepsilon} \cap(V-\Sigma(V)),
$$

será uma variedade suave $K$. Se $\varepsilon$ é suficientemente pequeno, então $S_{\varepsilon}$ não intersecta $\Sigma(V)$, portanto $K$ será igual a $S_{\varepsilon} \cap V$. O caso complexo é análogo pensando uma variedade complexa em $\mathbb{C}^{m}$ como uma variedade real em $\mathbb{R}^{2 m}$.

Definição 3.22 : Uma partição da unidade suave de um conjunto $K$ é uma família de funções $\left\{\lambda_{\alpha}\right\}$ suaves em $K$, tal que

$$
\lambda_{\alpha}(x) \geq 0, \quad \sum_{\alpha} \lambda_{\alpha}(x)=1, \quad \forall x \in K
$$

e, para cada ponto $x \in K$, existe uma vizinhança de $x$ onde apenas um número finito dos $\lambda_{\alpha}$ são não nulas.

Denotaremos por cone $(K)$ o cone sobre $K$ de vértice $x_{0}$, isto é

$$
\operatorname{cone}(K)=t k+(1-t) x_{0}, \quad \forall k \in K
$$

Seja $D_{\varepsilon}$ o disco fechado dos pontos $x$ satisfazendo $\left\|x-x_{0}\right\| \leq \varepsilon$, e seja $x_{0}$ um ponto regular ou um ponto singular isolado de $V$.

Teorema 3.23 : Para $\varepsilon$ pequeno, a interseção de $V$ com $D_{\varepsilon}$ é homeomorfa ao cone 
sobre $K=V \cap S_{\varepsilon}$. Na verdade, o par $\left(D_{\varepsilon}, V \cap D_{\varepsilon}\right)$ é homeomorfo ao par do cone sobre $S_{\varepsilon}$ e o cone sobre $K$.

Demonstração: É suficiente mostrar o caso real. Seja $\varepsilon>0$ suficientemente pequeno para que o disco $D_{\varepsilon}$ não contenha pontos singulares de $V$ e pontos críticos de $\left.r\right|_{V-\Sigma(V)}$, além de $x_{0}$. Vamos construir um campo suave $v$ (a princípio localmente) no disco perfurado $D_{\varepsilon}-x_{0}$ satisfazendo as seguintes propriedades:

i. $v(x)$ está sempre "saindo" de $x_{0}$,

ii. $v(x)$ é tangente à variedade $M_{1}=V-\Sigma(V)$, sempre que $x \in M_{1}$.

Dado $x_{\alpha} \in D_{\varepsilon}-x_{0}$, vamos construir um campo $V_{\alpha}(x)$ em uma vizinhança $U_{\alpha}$ de $x_{\alpha}$ que satisfaz as propriedades acima.

Se $x_{\alpha}$ não pertence à $V$, tome $v_{\alpha}(x)=x-x_{0}$, para todo $x \in U_{\alpha} \subset \mathbb{R}^{n}-V$.

Se $x_{\alpha} \in V$, então $x_{\alpha} \in M_{1}$. Assim, como antes, podemos escolher coordenadas $u_{1}, \ldots, u_{n}$ para uma vizinhança $U_{\alpha}$ de $x_{\alpha}$, tal que $M_{1}$ corresponde localmente a $u_{1}=$ $\ldots=u_{\rho}=0$. Assim, como $x_{\alpha}$ não é ponto crítico de $\left.r\right|_{M_{1}}$, onde $r(x)=\left\|x-x_{0}\right\|^{2}$, temos que as últimas derivadas

$$
\frac{\partial r}{\partial u_{\rho+1}}, \ldots, \frac{\partial r}{\partial u_{n}}
$$

devem ser não nulas em $x_{\alpha}$.

Suponha $\frac{\partial r}{\partial u_{h}}\left(x_{\alpha}\right) \neq 0$, seja $U_{\alpha}$ uma vizinhança conexa tal que $\frac{\partial r}{\partial u_{h}}(x) \neq 0, \forall x \in U_{\alpha}$. Seja $v_{\alpha}(x)$ o vetor

$$
\pm\left(\frac{\partial x_{1}}{\partial u_{h}}, \ldots, \frac{\partial x_{m}}{\partial u_{h}}\right)
$$

que é tangente à $u_{h}$-curva coordenada em $x$. O sinal \pm é escolhido de acordo com $\frac{\partial r}{\partial u_{h}}$ ser positivo ou negativo. Este vetor $v_{\alpha}(x)$ é tangente à $M_{1}$, sempre que $x \in M_{1}$, visto que a $u_{h}$-curva coordenada está contida em $M_{1}$. Além disso, como $r^{\prime}(x)=2\left(x-x_{0}\right)$, 
temos

$$
\begin{aligned}
2\left\langle v_{\alpha}(x), x-x_{0}\right\rangle & =\sum v_{\alpha, i}(x) \cdot 2\left(x_{i}-x_{0, i}\right) \\
& =\sum\left( \pm \frac{\partial x_{i}}{\partial u_{h}}\right)\left(\frac{\partial r}{\partial x_{i}}\right) \\
& = \pm \frac{\partial r}{\partial u_{h}}, \quad \forall x \in U_{\alpha} .
\end{aligned}
$$

Escolha uma partição da unidade suave $\left\{\lambda_{\alpha}\right\}$ em $D_{\varepsilon}-x_{0}$, com $\operatorname{supp}\left(\lambda^{\alpha}\right) \subset U^{\alpha}$. Assim, o campo de vetores

$$
v(x)=\sum_{\alpha} \lambda_{\alpha}(x) v_{\alpha(x)}
$$

satisfaz as propriedades $i$. e $i i$. em $D_{\varepsilon}-x_{0}$.

Normalize o campo, fazendo

$$
w(x)=\frac{v(x)}{\|v(x)\|}
$$

e considere a equação diferencial

$$
\frac{d x}{d t}=w(x)
$$

Isto é, olhe para as curvas suaves $x(t)=\rho(t)$ definidas em $\alpha<t<\beta$, que satisfazem

$$
\frac{d \rho(t)}{d t}=w(\rho(t))
$$

Dada uma solução $\rho(t)$, a derivada da função composta $r(\rho(t))$ nos dá

$$
\begin{aligned}
\frac{d r}{d t} & =\sum\left(\frac{\partial r}{\partial x_{i}}\right) \cdot w_{i}(x) \\
& =\left\langle 2\left(x-x_{0}\right), w(x)\right\rangle=1
\end{aligned}
$$

onde $x=\rho(t)$. Assim, a função $r(\rho(t))$ deve ser igual a $t$ mais uma constante. Fazendo uma mudança de parâmetros, podemos assumir que

$$
r(\rho(t))=\left\|\rho(t)-x_{0}\right\|^{2}=t
$$


Esta solução pode ser estendida ao intervalo $0<t \leq \varepsilon^{2}$. De fato, podemos supor que $w$ foi construído em um aberto um pouco maior que $D_{\varepsilon}-x_{0}$, deste modo, os pontos de fronteira não serão problema. Pelo Lema de Zorn, uma solução $\rho(t)$ pode ser extendida a um intervalo maximal $\alpha^{\prime}<t<\beta^{\prime}$. Suponha $\beta \leq \varepsilon^{2}$. Vamos mostrar que é possível extender $\rho$ a um intervalo um pouco maior, contradizendo a definição de $\beta^{\prime}$. Como $\rho(t) \in D_{\varepsilon}, \forall t \in\left(\alpha^{\prime}, \beta^{\prime}\right)$ e $D_{\varepsilon}$ é compacto, temos que existe $x^{\prime} \in D_{\varepsilon}$ tal que $x^{\prime}$ é o limite da sequência $\{\rho(t)\}$ quando $t$ tende à $\beta^{\prime}$. Pela continuidade de $\rho$, temos $\rho\left(x^{\prime}\right)=\beta^{\prime} \neq 0$ e, portanto, $x^{\prime} \in D_{\varepsilon}-x_{0}$. Pelo teorema de existência e unicidade (e suavidade) da equação diferencial $\frac{d x}{d t}=w(x)$ na vizinhança de $x^{\prime}$ temos que, para cada $x^{\prime \prime}$ em uma vizinhança $U$ de $x^{\prime}$ e cada $t^{\prime \prime}$ em um intervalo arbitrariamente pequeno $I$ contendo $\beta^{\prime}$, existe uma única solução $x=q(t)$, com $t \in I$, satisfazendo a condição inicial $q\left(t^{\prime \prime}\right)=x^{\prime \prime}$. Além disso, o teorema garante que $q(t)$ é uma função suave de $x^{\prime \prime}$, $t^{\prime \prime}$ e $t$. Pela unicidade, temos que $p(t)=q(t)$ em $I^{\prime}\left(\alpha^{\prime}, \beta^{\prime}\right) \cap I$. Escolha $t^{\prime \prime} \in I^{\prime}$ e seja $x^{\prime \prime}=p\left(t^{\prime \prime}\right)$. Assim, podemos definir a solução $p(t)$ ao intervalo $I^{\prime}$, tomando a solução $q(t)$. O que contradiz o fato de $\beta^{\prime}$ ser maximal. Analogamente podemos provar que $\alpha^{\prime}=0$. Note que a solução $\rho(t)$ é únicamente determinada pelo valor inicial $\rho\left(\varepsilon^{2}\right) \in S_{\varepsilon}$. Assim, para cada $a \in S_{\varepsilon}$, seja

$$
p(t)=P(a, t), \quad 0<t \leq \varepsilon^{2}
$$

a única solução satisfazendo a condição inicial

$$
p\left(\varepsilon^{2}\right)=P(a, \varepsilon)=a .
$$

Temos então que $P$ leva difeomorficamente o disco perfurado $D-x_{0}$ no cilindro $S_{\varepsilon} \times$ $\left(0, \varepsilon^{2}\right]$. Além disso, como $w(x)$ é tangente a $M_{1}$ sempre que $x \in M_{1}$, temos que toda curva solução que intersecta $M_{1}$ deve estar contida em $M_{1}$. Logo, $P$ leva $K \times\left(0, \varepsilon^{2}\right]$ difeomorficamente em $V \cap\left(D_{\varepsilon}-x_{0}\right)$. 


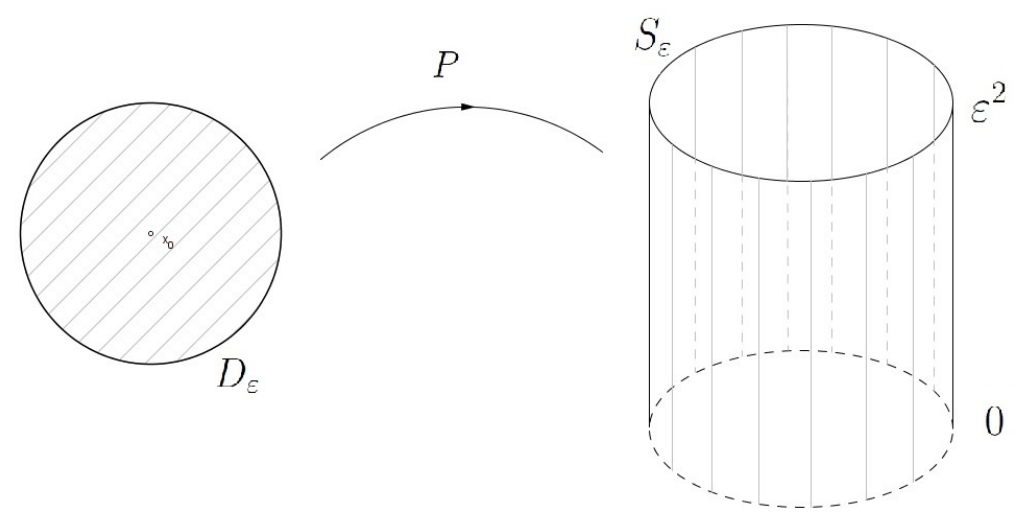

Figura 3.3: Aplicação $P$

Note que $P(a, t)$ tende uniformemente a $x_{0}$ quando $t \rightarrow 0$. Portanto a correspondência

$$
t a+(1-t) x_{0} \mapsto P\left(a, t \varepsilon^{2}\right)
$$

definida para $0<t \leq 1$, se estende unicamente a um difeomorfismo do $\operatorname{Cone}\left(S_{\varepsilon}\right)$ em $D_{\varepsilon}$. Além disso, este difeomorfismo leva o Cone $(K)$ em $V \cap D_{\varepsilon}$.

O teorema a seguir é o principal resultado de [20] e estabelece uma relação entre a topologia do link $K$ e $V$.

Teorema 3.24 (Fibração de Milnor): Se z é um ponto qualquer de $V$ e se $S_{\varepsilon}$ é uma esfera suficientemente pequena centrada em $z_{0}$, então $S_{\varepsilon}-K$ é um fibrado diferenciável localmente trivial sobre $S^{1}$, com projeção $\phi(z)=\frac{f(x)}{|f(z)|}$ e fibra $F_{\theta}=\phi^{-1}\left(e^{i \theta}\right)$.

Os resultados de Milnor sobre a topologia da fibra de Milnor $F_{\theta}$ são descritos a seguir, e a referência também é [20]

Para o caso particular em que $f$ tem uma singularidade isolada em 0 , o teorema abaixo caracteriza o tipo de homotopia da fibra $F_{\theta}$.

Teorema 3.25 Cada fibra $F_{\theta}$ tem o mesmo tipo de homotopia de um bouquet $S^{n-1} \vee$ $\cdots \vee S^{n-1}$ de esferas de dimensão $n-1$ em $\mathbb{K}^{n}$. O número de Milnor é o número de esferas neste bouquet. 


\subsection{Conjuntos Analíticos}

Definição 3.26 : Seja $f: \mathbb{C}^{n+1} \rightarrow \mathbb{C}$. Dizemos que $f$ é holomorfa em a $\in \mathbb{C}^{n+1}$ se existe aberto $U \subset \mathbb{C}^{n+1}$ tal que $f$ é diferenciável (no sentido complexo, isto é, diferenciável com derivada $\mathbb{C}$-linear) em todo ponto $u \in U$.

Assim como no capítulo anterior, vamos estudar o conjunto de zeros de funções, agora não mais polinomiais, mas analíticas. Estes conjuntos são chamados de espaços analíticos

Definição 3.27 Considere o conjunto dos pares $\left(V_{\alpha}, U_{\alpha}\right)$, onde $U_{\alpha}$ é uma vizinhança aberta da origem e $V_{\alpha}$ é subconjunto de $U_{\alpha}$. Dois pares $\left(V_{1}, U_{1}\right)$ e $\left(V_{2}, U_{2}\right)$ são equivalentes se existe uma vizinhança $W \subset U_{1} \cap U_{2}$ tal que $V_{1} \cap W=V_{2} \cap W$. A classe de equivalência de um par é chamada de germe na origem em $\mathbb{C}^{n}$.

Se $f \in \mathcal{O}_{n}$, a classe de equivalência do conjunto $\{x ; f(x)=0\}$, com $f$ um representante do germe $f$, é denotada por $V(f)$. Assim como anteriormente, se $f_{1}$ e $f_{2}$ são representantes de um mesmo germe, então $V\left(f_{1}\right)=V\left(f_{2}\right)$.

Definição 3.28 Um germe de espaço analítico $(\mathcal{V}, 0)$ em torno da origem é o germe do subconjunto

$$
\mathcal{V}=V\left(f_{1}, f_{2}, \ldots, f_{r}\right)=V\left(f_{1}\right) \cap V\left(f_{2}\right) \cap \ldots \cap V\left(f_{r}\right), \quad f_{1}, f_{2}, \ldots, f_{r} \in \mathcal{O}_{n}
$$

O ideal de um germe de espaço analítico $\mathcal{V}$ é definido por

$$
\mathcal{I}(\mathcal{V})=\left\{f \in \mathcal{O}_{n} ; \mathcal{V} \subset f^{-1}(0)\right\}
$$

Definição 3.29 : Um germe de espaço analítico é irredutível se para $V_{1}$ e $V_{2}$ germes quaisquer, com $\mathcal{V}=V_{1} \cup V_{2}$, tem-se que $\mathcal{V}=V_{1}$ ou $\mathcal{V}=V_{2}$. Neste caso, $\mathcal{V}$ é uma variedade analítica. 
Proposição 3.30 : Seja $\mathcal{V}$ um germe de espaço analítico. Então existem p um número inteiro positivo e $V_{1}, \ldots, V_{p}$ variedades irredutiveis, com $V_{i} \nsubseteq V_{j}$, sempre que $i \neq j$, tais que $\mathcal{V}=V_{1} \cup \ldots \cup V_{p}$. Essas variedades são unicamente determinadas, a menos da ordem, e são chamadas de componentes irredutiveis de $\mathcal{V}$.

Definição 3.31 : Um germe de espaço analítico $\mathcal{V}$ é equidimensional se todas as suas componentes irredutíveis tem a mesma dimensão.

Um germe de espaço analítico em $x$ é um germe de conjunto $\mathcal{V}$ em $x$ tal que, para alguma vizinhança $U$ de $x$, o germe $\mathcal{V} \cap U$ pode ser descrito por $V\left(f_{1}, \ldots, f_{r}\right)$, para alguns $f_{1}, \ldots, f_{r} \in \mathcal{O}_{n}$.

Definição 3.32 : Seja I um anel. Um elemento a $\in I$ é nilpotente se existe $n \in \mathbb{N}$ tal que $a^{n}=0$.

Definição 3.33 Dizemos que um germe $\mathcal{V}=V\left(f_{1}, \ldots, f_{r}\right)$ é reduzido a $\mathbb{C}$-álgebra $\frac{\mathcal{O}_{n}}{\left\langle f_{1}, \ldots, f_{r}\right\rangle}$ não possui elementos nilpotentes.

Um ponto $z$ de um germe de espaço analítico $\mathcal{V}$ é um ponto regular ou suave se para alguma vizinhança $U$ de $z$ o germe $U \cap \mathcal{V}$ pode ser descrito como o conjunto dos zeros de um número finito de funções analíticas que possuem $z$ como ponto regular. Um ponto de $\mathcal{V}$ não regular é chamado ponto singular de $\mathcal{V}$. 


\section{Capítulo}

\section{Índices de Campos de Vetores: Caso}

Singular

Neste capítulo estudaremos o índice de Schwartz e o índice GSV de campos de vetores em variedades singulares. As principais referências são o livro [3] e a dissertação de mestrado [4].

Definição 4.1 : Seja $V$ um espaço topológico. Dizemos que $V$ é uma variedade singular $n$-dimensional se existe um subconjunto $S \subset V$, tal que, $V-S$ é uma variedade topológica n-dimensional, e tal que, $\forall a \in S$, não existe vizinhança $V_{\alpha}$ de a em $V$ homeomorfa ̀̀ um aberto $U \subset \mathbb{R}^{m}$.

Definição 4.2 : Seja $V$ uma variedade singular $n$-dimensional. Chamamos de conjunto singular de $V$ o subconjunto $S$ da definição acima, e denotamos por $\Sigma V$ tal conjunto. Cada ponto em $S$ é dito uma singularidade de $V$ e a singularidade a $\in S$ é dita isolada se existe aberto $W_{a} \subset V$ tal que $W_{a} \cap S=a$.

Exemplo 4.3 : O toro pinçado e a figura oito são exemplos de variedades singulares, 2-dimensional e 1-dimensional, respectivamente.

Observação 4.4 : Vamos tratar apenas das variedades analíticas. Assim, o conjunto $V_{\text {reg }}=V-\Sigma V$ é uma variedade diferenciável. 
Definição 4.5 : Seja $V$ uma variedade mergulhada em $\mathbb{R}^{n}$ com uma singularidade isolada $a \in V$. Um campo contínuo $v$ em $V$ é uma aplicação contínua $v: V \rightarrow \mathbb{R}^{n}$, tal que $v(x) \in T_{x} V_{\text {reg }}, \forall x \in V_{\text {reg }}$ e $v(a)=0$.

Definição 4.6 : Considere em $V$ um campo contínuo $v$, tal que $v$ possa ser definido sobre uma bola fechada no espaço ambiente $\overline{B_{\varepsilon}(a)} \subset \mathbb{R}^{n}$, com singularidade isolada no ponto a de $V$ em $\overline{B_{\varepsilon}(a)}$. Definimos o índice de Poincaré-Hopf de $v$ em a como sendo o indice de Poincaré-Hopf de $\widetilde{v}$, onde $\widetilde{v}=\left.v\right|_{\overline{B_{\varepsilon}(a)}}$.

Exemplo 4.7 : Considere os seguintes campos sobre o toro pinçado $V$ (figuras 4.1 e 4.2)

Através de uma triangulação, pode-se provar que $\chi(V)=1$. Logo, o teorema de Poincaré-Hopf não se verifica para o campo $w$, entretanto se verifica para o campo $v$.

Exemplo 4.8 : Considere o campo $v$ sobre a figura oito, como na figura 4.3

Então, sobre os pontos $a, b_{1}, b_{2}, b_{3}$ e $b_{4}$, teremos os campos

Assim, somando os índices, temos -1, que é a característica de Euler da figura 8.

\section{1 Índice de Schwartz}

Seja $V \subset \mathbb{C}^{m}$ um espaço analítico equidimensional de dimensão maior ou igual a 1 , com singularidade isolada em $0 \in \mathbb{C}^{m}$. Seja $\overline{B_{\varepsilon}(0)}$ a bola fechada de centro em 0 e raio $\varepsilon>0$ suficientemente pequeno para que toda esfera centrada em 0 , contida em $\overline{B_{\varepsilon}(0)}$, encontre $V$ transversalmente (o que é possível pelo capítulo anterior).

Definição 4.9 Seja $v_{\text {rad }}$ um campo de vetores em $V-\{0\}$ que seja transversal a todas as esferas $S_{\varepsilon^{\prime}}$, para $\varepsilon>\varepsilon^{\prime}>0$ suficientemente pequeno, que aponta para fora em 0 e que se estenda continuamente em $\mathbb{C}^{m}$ com singularidade isolada em 0 . Chamamos de $v_{\text {rad }}$ o campo radial em $V$.

Exemplo 4.10 : O campo $v$ do Exemplo 4.7 sobre o toro pinçado é um exemplo de campo radial. 


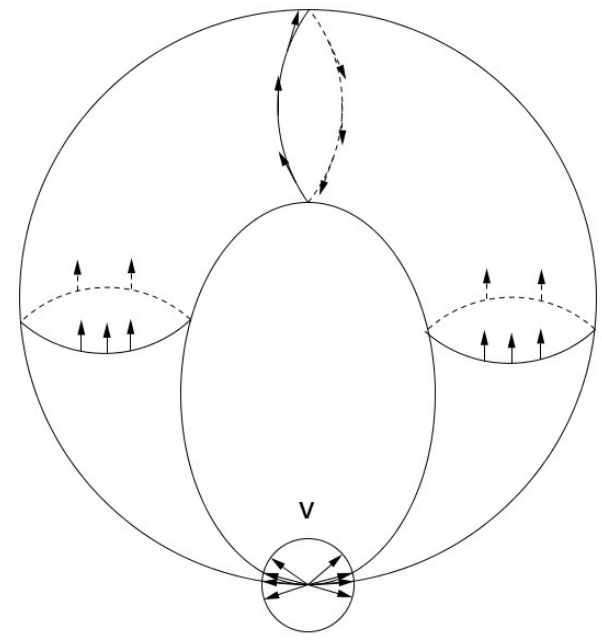

Figura 4.1: Campo v

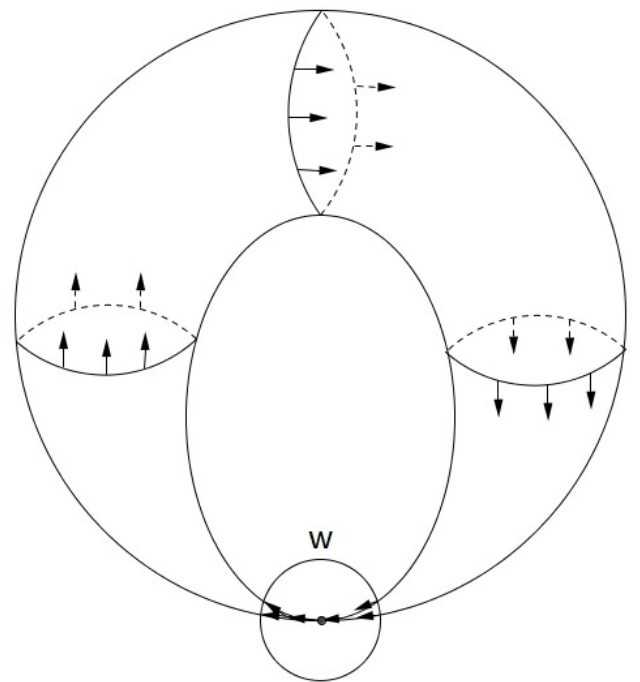

Figura 4.2: Campo w

Observação 4.11 : O campo $v_{\text {rad }}$ pode ser estendido a um campo tangente à $V$ em $\overline{B_{\varepsilon}(0)}$, o qual denotaremos por $v_{r a d}^{\#}$, sendo transversal à todas as esferas $S_{\varepsilon^{\prime}}$, com $\varepsilon \geq$ $\varepsilon^{\prime}>0$, obtendo assim um campo radial para $\overline{B_{\varepsilon}(0)}$. Este campo $v_{\text {rad }}^{\#}$ é chamado extensão radial do campo $v$.

Definição 4.12 : O indice de Schwartz de $v_{\text {rad }}$ é o indice de Poincaré-Hopf da extensão radial $v_{\text {rad }}^{\#}$, o qual é +1 .

Queremos agora definir o índice de Schwartz para um campo contínuo $v$ em $V$, com singularidade isolada em 0, qualquer. Para isso, precisamos definir a diferença entre o 


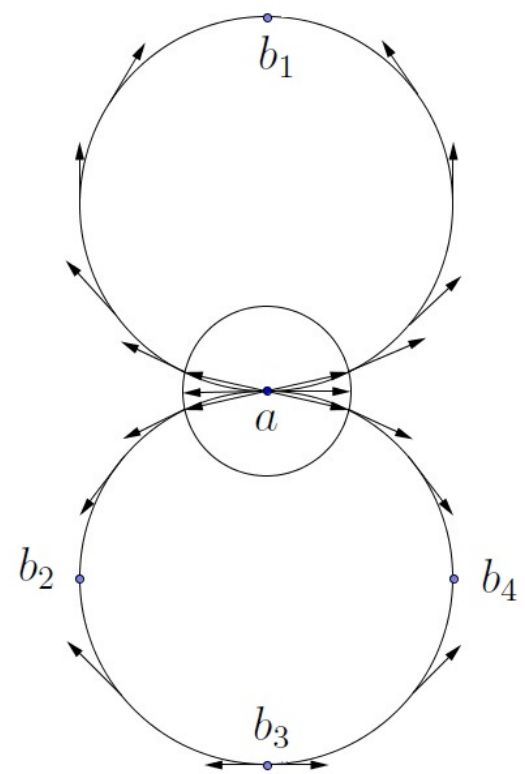

Figura 4.3: figura 8

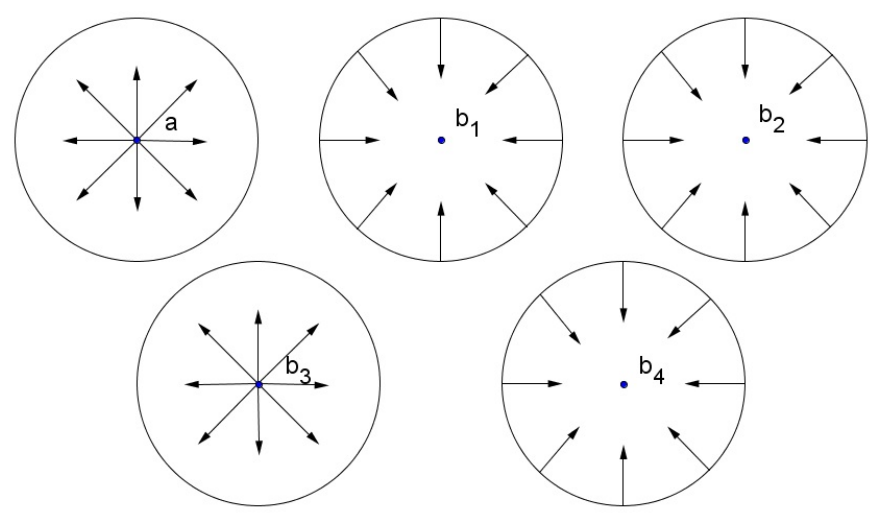

Figura 4.4: Campos sobre a figura 8

campo $v$ e o campo $v_{r a d}$. A extensão de campos é garantida pelo teorema de Tietze.

Considere duas pequenas esferas $S_{\varepsilon}$ e $S_{\varepsilon^{\prime}}$, com $\varepsilon>\varepsilon^{\prime}>0$, centradas em 0. Seja $X$ o cilindro em $V$ limitado pelos links

$$
K_{\varepsilon}=S_{\varepsilon} \cap V \quad e \quad K_{\varepsilon^{\prime}}=S_{\varepsilon^{\prime}} \cap V .
$$


Sobre $X$, considere um campo $w$ que possui uma quantidade finita de singularidades no interior de $X$ e que satisfaz

$$
\left.w\right|_{K_{\varepsilon}}=v \quad \text { e }\left.\quad w\right|_{K_{\varepsilon^{\prime}}}=v_{\text {rad }} .
$$

Definição 4.13 : A diferença entre $v$ e $v_{\text {rad }}$ é definida como

$$
d\left(v, v_{r a d}\right)=\operatorname{Ind}_{P H}(w, X) .
$$

Definição 4.14 : Sejam $V \subset \mathbb{C}^{m}$ um espaço analítico, equidimensional, com singularidade isolada em 0 e $v$ um campo de vetores contínuo em $V$, com singularidade isolada em 0. Definimos o indice de Schwartz do campo $v$ em 0 por

$$
\operatorname{Ind}_{S c h}(0, v ; V)=1+d\left(v, v_{r a d}\right) .
$$

O teorema a seguir é a versão do Teorema de Poincaré-Hopf para variedades com singularidades isoladas.

Teorema 4.15 : Seja $V$ um espaço analítico, equidimensional e compacto, contido em $\mathbb{C}^{m}$, com singularidades isoladas $q_{1}, \ldots, q_{r}$. Seja $v$ um campo de vetores contínuo em $V$, com singularidades em $q_{1}, \ldots, q_{r}$ e, possivelmente também nos pontos $p_{1}, \ldots, p_{s}$. Seja

$$
\operatorname{Ind}_{S c h}(v, V)=\sum_{i=1}^{r} \operatorname{Ind}_{S c h}\left(v, q_{i} ; V\right)+\sum_{j=1}^{s} \operatorname{Ind}_{P H}\left(v, p_{i}\right) .
$$

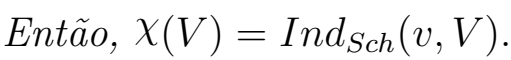

Demonstração: Suponha $v$ radial em $q_{1}, \ldots, q_{r}$. Então

$$
\operatorname{Ind}_{S c h}\left(v, q_{i} ; V\right)=1, \quad \forall i=1, \ldots, r .
$$

Em cada ponto $q_{i}$, considere a bola fechada $\overline{B_{\varepsilon_{i}}\left(q_{i}\right)} \subset \mathbb{C}^{m}$, com $\varepsilon_{i}$ suficientemente pequeno para que $q_{i}$ seja a única singularidade de $v$ em $\overline{B_{\varepsilon_{i}}\left(q_{i}\right)} \cap V$. Defina $D_{i}=$ 
$V \cap \overline{B_{\varepsilon_{i}}\left(q_{i}\right)}$. Assim, temos que

$$
V^{*}=V-\bigcup_{i=1}^{r} \operatorname{int}\left(D_{i}\right)
$$

é uma variedade diferenciável com bordo (basta notar que a aplicação $\rho(x)=\|x\|^{2}$ é uma submersão fora da origem, restrita a $\left.V^{*}\right)$.

Como o campo $v$ é transversal ao bordo de $V^{*}$, temos

$$
\chi\left(V^{*}\right)=\sum_{j=1}^{s} \operatorname{Ind}_{P H}\left(v, p_{j}\right)
$$

Sabendo que $\chi(V)=\chi\left(\bigcup_{i=1}^{r} D_{i}\right)+\chi\left(V^{*}\right)$, temos que

$$
\chi(V)=\sum_{i=1}^{r} \operatorname{Ind}_{S c h}\left(v, q_{i}\right)+\sum_{j=1}^{s} \operatorname{Ind}_{P H}\left(v, p_{j}\right) .
$$

Note que a primeira somatória se deve ao fato do campo $v$ ser radial em cada $q_{i}$.

Por outro lado, suponha que $v$ não seja radial em algum $q_{i}$ fixado. Seja $\varepsilon>0$ suficientemente pequeno. Considere $\overline{B_{i, \varepsilon}\left(q_{i}\right)}$ a bola fechada de centro em $q_{i}$ e raio $\varepsilon$, em $\mathbb{C}^{m}$. Seja $S_{i, \varepsilon}$ a esfera de mesmo raio e centro que $\overline{B_{i, \varepsilon}\left(q_{i}\right)}$. Sejam

$$
K_{i, \varepsilon}=V \cap S_{i, \varepsilon} \quad e \quad V^{*}=V-\left(V \cap \overline{B_{i, \varepsilon}\left(q_{i}\right)}\right)
$$

Pelo teorema 3.23, temos que cada $K_{i, \varepsilon}$ possui uma vizinhança difeomorfa ao cilindro $K_{i, \varepsilon} \times[0,1]$. Tomemos $\varepsilon>\varepsilon_{1}>\varepsilon_{2}>0$ e sejam $X_{\varepsilon_{1}}, X_{\varepsilon_{2}}$ os cilindros limitados por $\left\{K_{i, \varepsilon}, K_{i, \varepsilon_{1}}\right\}$ e $\left\{K_{i, \varepsilon}, K_{i, \varepsilon_{2}}\right\}$, respectivamente.

Coloque o campo $v$ sobre $K_{i, \varepsilon_{1}}$ e o campo radial sobre $K_{i, \varepsilon_{2}}$ e $K_{i, \varepsilon}$, chamemos este campo de $w$. Temos que

$$
\operatorname{Ind}_{S c h}\left(v, q_{i}\right)=1+d\left(v, v_{r a d}\right)
$$




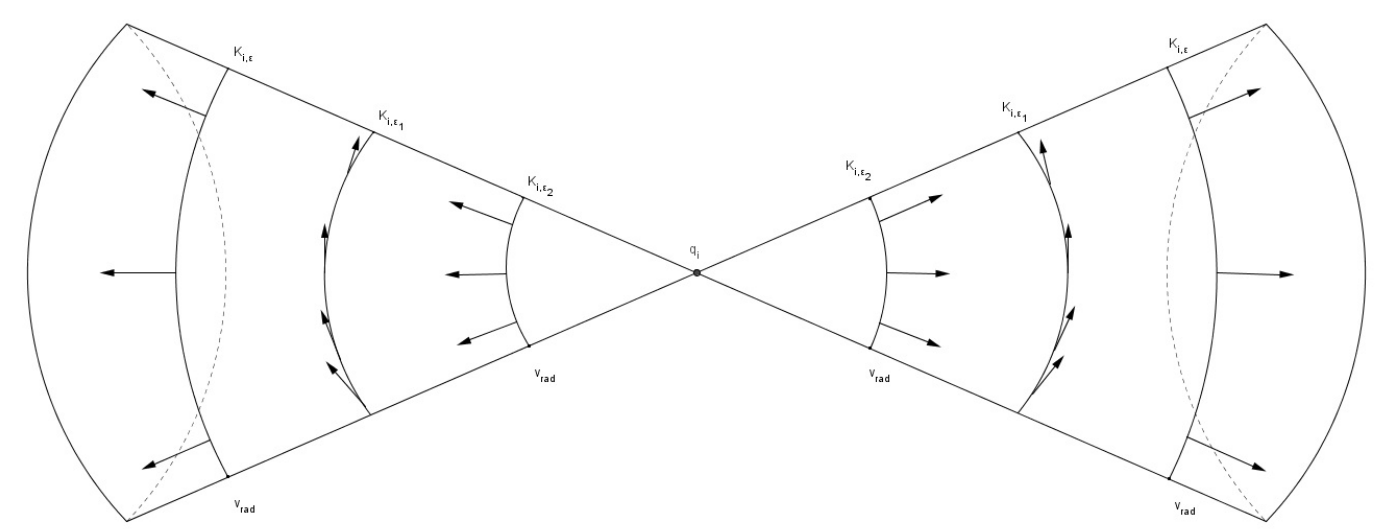

Figura 4.5: Campo radial e o campo $v$ sobre a variedade $V$

Considere a extensão contínua de $w$ sobre os cilindros $X_{\varepsilon_{1}}$ e $X_{\varepsilon_{2}}$, chamemos este campo de $\widetilde{w}$. Como $X_{\varepsilon_{1}}$ e $X_{\varepsilon_{2}}$ são cilindros, é possível estender $w$ à $\widetilde{w}$ sem singularidades, pelo Teorema de Tietze. Assim, por (1) e (2), temos que

$$
d\left(v_{r a d}, v\right)+d\left(v, v_{r a d}\right)=0 .
$$

Logo, voltamos ao caso anterior.

\subsection{Exemplos}

Denotaremos por $(V, 0)$ o germe de uma hipersuperfície analítica complexa, dada por uma função holomorfa

$$
f:\left(\mathbb{C}^{n+1}, 0\right) \rightarrow(\mathbb{C}, 0)
$$

a qual é definida em uma pequena bola $B_{\varepsilon}$ e que tem um único ponto crítico em 0 . Seja $V^{*}=V-\{0\}$. O espaço tangente a um ponto $x \in V^{*}$ será o conjunto

$$
T_{x} V^{*}=\left\{\xi \in T_{x} \mathbb{C}^{n+1} ; d f_{x}(\xi)=0\right\}=\operatorname{ker}\left(d f_{x}\right)
$$

Exemplo 4.16: Seja $f: \mathbb{C}^{2} \rightarrow \mathbb{C}$ dada por $f(u, v)=u^{2}+v^{3}$. Temos que $f$ é 
polinomial, logo é holomorfa. Além disso, $\nabla f=\left(2 u, 3 v^{2}\right)$, portanto, $0 \in \mathbb{C}^{2}$ é a única singularidade de $f$.

Afirmação: Se $z=(u, v) \in V^{*}$, então a reta tangente em $z$ é gerada por $\xi(u, v)=$ $\left(3 v^{2},-2 u\right)$.

De fato, temos

$$
d f(\xi)=2 u \xi_{1}+3 v^{2} \xi_{2}=0 \quad \Rightarrow \quad 2 u \xi_{1}=-3 v^{2} \xi_{2} .
$$

Como $(u, v) \neq 0$, podemos supor $v \neq 0$. Assim, $\xi_{2}=\frac{-2 u \xi_{1}}{3 v^{2}}$. Tomando $\xi_{1}=3 v^{2}$, temos $\xi_{2}=-2 u$.

Vamos interpretar agora um campo contínuo de vetores $v:(V, 0) \rightarrow\left(\mathbb{C}^{n+1}, 0\right)$ como uma aplicação contínua cuja imagem está contida no tangente a $V$ em cada ponto, isto é, $v(x) \in T_{x} V^{*}$, para $x \in V^{*}$. Ou ainda, $v(x) \in \operatorname{Ker}\left(d f_{x}\right)$. Como $V \subset B_{\varepsilon}(0)$ é fechado, novamente pelo teorema de Tietze, $v$ se estende a uma vizinhança de $V$ em $\mathbb{C}^{n+1}$. Em outras palavras, um campo sempre pode ser estendido ao espaço ambiente, bem como ser considerado como restrição de um.

O seguinte resultado pode ser encontrado em [1].

Teorema 4.17 : Seja $V$ uma variedade analítica complexa em $\mathbb{C}^{m}$ com singularidade isolada em 0. Então:

i. O espaço de campos holomorfos em $V$ com singularidade isolada em 0 têm dimensão infinita.

ii. Se v é um campo holomorfo com singularidade isolada em 0 , então existem infinitas extensões de $v$ a uma vizinhança de $0 \mathrm{em} \mathbb{C}^{m}$.

Exemplo 4.18: Seja $(V, 0)$ o germe de $f:\left(\mathbb{C}^{2}, 0\right) \rightarrow(\mathbb{C}, 0)$, onde $f$ possui uma singularidade isolada em 0 . Dados $\bar{\xi}=\left(-\frac{\partial f}{\partial z_{2}}, \frac{\partial f}{\partial z_{1}}\right)$ e $w \in V^{*}$, temos

$d f(\bar{\xi}(w))=d f\left(-\frac{\partial f}{\partial z_{2}}(w), \frac{\partial f}{\partial z_{1}}(w)\right)=-\frac{\partial f}{\partial z_{1}}(w) \cdot \frac{\partial f}{\partial z_{2}}(w)+\frac{\partial f}{\partial z_{2}}(w) \cdot \frac{\partial f}{\partial z_{1}}(w)=0$. 
Logo, $\bar{\xi}$ é um campo tangente à $V$ com uma única singularidade isolada em 0 . Além disso, dado $t \in \mathbb{C}$, temos que $\bar{\xi}$ é tangente à fibra $f^{-1}(t)$, pois $(*)$ não depende da escolha de $w$. Considere $\xi$ a restrição de $\bar{\xi} \grave{a} V$. Seja g uma função holomorfa em $\mathbb{C}^{2}$ tal que $V=g^{-1}(0)$ e que representa um elemento não nulo no anel local $\mathrm{O}_{2}$, então

$$
\beta=\left(g-\frac{\partial f}{\partial z_{2}}, g+\frac{\partial f}{\partial z_{1}}\right),
$$

coincide com $\xi$ em $V$, mas não é tangente à todas as fibras de $f$ pois, dado $w \in f^{-1}(t)$, com $t \neq 0$, temos

$$
\begin{aligned}
d f_{w}(\beta(w)) & =d f_{w}\left(g(w)-\frac{\partial f}{\partial z_{2}}, g(w)+\frac{\partial f}{\partial z_{1}}(w)\right) \\
& =\frac{\partial f}{\partial z_{1}} \cdot g(w)-\frac{\partial f}{\partial z_{1}}(w) \frac{\partial f}{\partial z_{2}}(w)+\frac{\partial f}{\partial z_{2}}(w) g(w)+\frac{\partial f}{\partial z_{2}}(w) \frac{\partial f}{\partial z_{1}}(w) \\
& =g(w)\left(\frac{\partial f}{\partial z_{1}}(w)+\frac{\partial f}{\partial z_{2}}(w)\right)
\end{aligned}
$$

Como $t \neq 0$, temos $g(w) \neq 0$. Além disso, como $\frac{\partial f}{\partial z_{1}}(w)$ e $\frac{\partial f}{\partial z_{2}}(w)$ são L.I., temos que a soma é diferente de zero, portanto $d f_{w}(\beta(w)) \neq 0$.

Exemplo $4.19:$ Sejam $f:\left(\mathbb{C}^{3}, 0\right) \rightarrow(\mathbb{C}, 0)$ uma função holomorfa com ponto crítico isolado $0 \in \mathbb{C}$ e $V=f^{-1}(0)$. Podemos fazer mudanças de coordenadas de tal forma que $V$ intersecta o conjunto onde as derivadas parciais de $f$ com relação às variáveis $z_{2}$ e $z_{3}$ são nulas somente em zero, isto é

$$
V \cap\left\{\frac{\partial f}{\partial z_{2}}, \frac{\partial f}{\partial z_{3}}\right\}=\{0\}
$$

Defina um campo holomorfo em $\mathbb{C}^{3}$, dado por

$$
\bar{\xi}=\left(f, \frac{\partial f}{\partial z_{3}},-\frac{\partial f}{\partial z_{2}}\right)
$$


Assim,

$$
\begin{aligned}
d f_{w}(\bar{\xi}(w)) & =d f_{w}\left(f(w), \frac{\partial f}{\partial z_{3}}(w),-\frac{\partial f}{\partial z_{2}}(w)\right) \\
& =\frac{\partial f}{\partial z_{1}}(w) \cdot f(w)+\frac{\partial f}{\partial z_{2}}(w) \cdot \frac{\partial f}{\partial z_{3}}(w)-\frac{\partial f}{\partial z_{2}}(w) \cdot \frac{\partial f}{\partial z_{3}}(w)=\frac{\partial f}{\partial z_{1}} \cdot f(w)
\end{aligned}
$$

De (1) e (2) temos que $\bar{\xi}$ tem singularidade isolada em 0 . Ainda de (2), temos que $d f_{w}(\bar{\xi})$ se anula onde $f$ se anula, logo $\bar{\xi}$ é tangente à $V$. Tomando $\xi=\left.\bar{\xi}\right|_{V}$, temos um campo sobre $V$ que é holomorfo, com singularidade isolada na origem e uma extensão em $\mathbb{C}^{3}$ que também possui uma singularidade isolada em 0 . Entretanto, $\bar{\xi}$ não é tangente à todas as fibras de $f$. Dado $w \in f^{-1}(t)$, com $t \neq 0$, temos

$$
d f_{w}(\bar{\xi}(w))=f(w) \cdot \frac{\partial f}{\partial z_{1}}(w) \quad \Rightarrow \quad\left(d f_{w}(\bar{\xi}(w))=0 \quad \Leftrightarrow \quad \frac{\partial f}{\partial z_{1}}(w)=0\right)
$$

Deste modo, temos que $\bar{\xi}$ é tangente as fibras de $f$ se, e somente se, $\frac{\partial f}{\partial z_{1}} \equiv 0$. Assim, teríamos $V\left(\frac{\partial f}{\partial z_{1}}\right)=\mathbb{C}^{3}$ e consequentemente

$$
\operatorname{dim}\left(V\left(\frac{\partial f}{\partial z_{1}}, \frac{\partial f}{\partial z_{2}}, \frac{\partial f}{\partial z_{3}}\right)\right)=1 \text { ou } 2
$$

O que é absurdo, pois $f$ tem singularidade isolada em 0. Agora, esquecendo que nos foi dado $\bar{\xi}$, considere apenas o campo $\xi$. Temos que, como $f$ se anula em $V$, $\xi$ é da forma $\xi=\left(0, \frac{\partial f}{\partial z_{3}},-\frac{\partial f}{\partial z_{2}}\right)$. Além disso, podemos estender $\xi$ a um campo holomorfo $\bar{\beta}$ em $\mathbb{C}^{3}$, dado por $\bar{\beta}=\left(0, \frac{\partial f}{\partial z_{3}},-\frac{\partial f}{\partial z_{2}}\right)$. Este campo $\bar{\beta}$ é tangente à todas as superfícies não singulares $f^{-1}(t)$. O conjunto singular de $\bar{\beta}$ é a curva $V\left(\frac{\partial f}{\partial z_{2}}, \frac{\partial f}{\partial z_{3}}\right)$, definida pelo ideal $I=\left(\frac{\partial f}{\partial z_{2}}, \frac{\partial f}{\partial z_{3}}\right)$. De fato, suponha que exista $g \in O_{3}$ tal que $\frac{\partial f}{\partial z_{2}}=g \cdot \frac{\partial f}{\partial z_{3}}$, assim,

$$
J(f)=\left\langle\frac{\partial f}{\partial z_{1}}, g \frac{\partial f}{\partial z_{3}}, \frac{\partial f}{\partial z_{3}}\right\rangle
$$

com $\operatorname{dim}\left(V\left(\frac{\partial f}{\partial z_{1}}, \frac{\partial f}{\partial z_{3}}\right)\right)=1$ ou 2 . Absurdo pois $f$ possui singularidade isolada na origem. 
Suponha que $\operatorname{dim}\left(V\left(\frac{\partial f}{\partial z_{2}}, \frac{\partial f}{\partial z_{3}}\right)\right)=2$. Novamente teremos dim $\left(V\left(\frac{\partial f}{\partial z_{1}}, \frac{\partial f}{\partial z_{2}}, \frac{\partial f}{\partial z_{3}}\right)\right)=$ 1 ou 2, o que é absurdo. Portanto $\operatorname{dim}\left(V\left(\frac{\partial f}{\partial z_{2}}, \frac{\partial f}{\partial z_{3}}\right)\right)=1$. Observe que $V\left(\frac{\partial f}{\partial z_{2}}, \frac{\partial f}{\partial z_{3}}\right)$ intersecta cada fibra não singular $f^{t}$ em um número finito de pontos, ou equivalentemente,

$$
\operatorname{dim}\left(V\left(\frac{\partial f}{\partial z_{2}}, \frac{\partial f}{\partial z_{3}}\right) \cap f^{-1}(t)\right)=0
$$

De fato, suponha que esta dimensão seja 1 e considere para $t \in \mathbb{C}^{*}$ a função a 1parâmetro

$$
\begin{aligned}
g_{t}:\left(\mathbb{C}^{3}, 0\right) & \rightarrow(\mathbb{C}, 0) \\
x & \mapsto f(x)-t
\end{aligned}
$$

Temos que $g_{t}^{-1}(0)=f^{-1}(t)$, logo

$$
\operatorname{dim}\left(V\left(\frac{\partial f}{\partial z_{1}}, \frac{\partial f}{\partial z_{2}}, \frac{\partial f}{\partial z_{3}}\right) \cap f^{-1}(t)\right)=\operatorname{dim}\left(V\left(\frac{\partial f}{\partial z_{1}}, \frac{\partial f}{\partial z_{2}}, \frac{\partial f}{\partial z_{3}}\right) \cap g_{t}^{-1}(0)\right)=0 \text { oи } 1 \text {. }
$$

Absurdo, pois $t \in \mathbb{C}^{*}$ é valor regular de $f$. Portanto, temos o desejado.

\subsection{O Índice GSV para Campos de Vetores em ICIS}

Definição 4.20 : Sejam $X$ uma subvariedade analítica complexa em $\mathbb{C}^{n}$ de dimensão $m$ e I um ideal que define $X$. Dizemos que I define uma interseção completa em 0 se $I$ admite $n-m$ geradores $\phi_{1}, \ldots, \phi_{n-m}$ em $\mathcal{O}_{n}$. Uma intersecção completa com singularidade isolada é denotada por ICIS.

Definição 4.21 : A Variedade de Stiefel $W_{k+1}(n+k)$ é o conjunto dos $k+1$ referenciais ortonormais em $\mathbb{R}^{n+k}$.

Seja $(V, 0)$ o germe de uma ICIS $n$-dimensional reduzida, com singularidade isolada em 0, definida pela aplicação holomorfa

$$
f=\left(f_{1}, \ldots, f_{k}\right):\left(\mathbb{C}^{n+k}, 0\right) \rightarrow\left(\mathbb{C}^{k}, 0\right) .
$$


Sejam $V^{*}=V-\{0\}$ e $v$ um campo contínuo sobre $V$ com singularidade isolada em 0 . Indiquemos por $\overline{\nabla f_{i}(x)}$ o conjugado do vetor gradiente $\nabla f_{i}(x)$ de $f_{i}, i=1, \ldots, k$. Então $\left\{v(x), \overline{\nabla f_{1}}(x), \ldots, \overline{\nabla f_{k}}(x)\right\}$ é um $k+1$ referencial, o qual podemos assumir, através de homotopia, ser um conjunto ortonormal. Deste modo, $v$ define uma aplicação de $V^{*}$ na variedade de Stiefel $W_{k+1}(n+k)$. Seja $K=S_{\varepsilon} \cap V$ o link de $V$ em 0 . Temos que $K$ é uma variedade orientada real de dimensão $2 n-1$ (ver [16], pg 81). Podemos definir então a seguinte aplicação contínua

$$
\Phi_{v}=\left(v, \overline{\nabla f_{1}}, \ldots, \overline{\nabla f_{k}}\right): K \rightarrow W_{k+1}(n+k) .
$$

Sobre as variedades de Stiefel, temos as seguintes propriedades (ver [10])

i. $W_{k+1}(n+k)$ é difeomorfa à $\frac{U(n+k)}{U(n-1)}$, onde $U(n)$ é o grupo das matrizes unitárias de dimensão $n \times n$.

ii. $W_{k+1}(n+k)$ é $(2 n-2)$-conexa, isto é, é conexa por caminhos e $\Pi_{i}\left(W_{k+1}(n+k)\right) \equiv$ $0,1 \leq i \leq 2 n-2$, onde $\Pi_{i}$ é o grupo de homotopia de grau $i$.

iii. $\Pi_{2 n-1}\left(W_{k+1}(n+k)\right) \cong \mathbb{Z}($ ver também [22]).

Deste modo, podemos concluir que $\Pi_{2 n-1}\left(W_{k+1}(n+k)\right)=H_{2 n-1}\left(W_{k+1}(n+k)\right)$ (ver Teorema de Hurewicz, em [9], pg. 366), ou seja, a homologia de $W_{k+1}(n+k)$ na dimensão $2 n-1$ é $\mathbb{Z}$. Vamos considerar agora o caso em que $K$ é conexa, caso contrário, considere apenas as componentes conexas. Então o grau de $\Phi_{v}$ está bem definido através do homeomorfismo induzido

$$
\left(\Phi_{v}\right)_{*}: H_{2 n-1}(K) \rightarrow H_{2 n-1}\left(W_{k+1}(n+k)\right),
$$

e denotaremos tal grau por $\operatorname{gr}\left(\Phi_{v}\right)$.

Definição 4.22 : O índice $G S V$ de $v$ em $0 \in V$, denotado por $\operatorname{Ind}_{G S V}(v, 0)$, é o grau da aplicação $\Phi_{v}$. 
Dada uma aplicação holomorfa $f: \mathbb{C}^{n+k} \rightarrow \mathbb{C}^{k}$, podemos considerar a fibração de Milnor associada à $f$ (referências [19] e [17]). A fibra de Milnor $F$ pode ser considerada uma variedade diferenciável, compacta, $2 n$ dimensional e com bordo $\partial F=K$. O campo $v$ pode ser considerado um campo não singular sobre $\partial F$

Teorema 4.23 : Nas condições acima, cada fibra $F_{f}$ tem o mesmo tipo de homotopia de um buquê de esferas $S^{n-1}$, e o número de esferas no buquê é igual ao número de Milnor $\mu$.

Uma referência para o teorema acima é [17].

Teorema 4.24 : Seja $(V, 0)$ o germe de uma ICIS n-dimensional reduzida, com uma singularidade isolada em 0 . Seja também $v$ um campo em $V$ com singularidade isolada em $0 \in V$. O indice $G S V$ possui as seguintes propriedades

(1) $\operatorname{Ind}_{G S V}(v, 0)=\operatorname{Ind}_{P H}(\widetilde{v}, F)$, onde $\widetilde{v}$ é a extensão de $v$ para o interior da fibra $F$

(2) Se v é transversal à $K$, então

$$
\operatorname{Ind}_{G S V}(v, 0)=1+(-1)^{n} \mu,
$$

onde $n=\operatorname{dim}_{\mathbb{C}} V$ e $\mu$ o número de Milnor de $f$ em 0 .

(3) $\mu=(-1)^{n} \cdot\left(\operatorname{Ind}_{G S V}(v, 0)-\operatorname{Ind} d_{S c h}(v, 0)\right)$, independentemente da escolha de $v$.

Demonstração: (1) Sabemos que o conjunto $\left\{\overline{\nabla f_{1}}, \ldots, \overline{\nabla f_{k}}\right\}$ é LI em $F$ e normal à $V$. Então o grau de $\Phi_{v}$ pode ser identificado como a obstrução de estender $v$ à um campo tangente em $F$.

(2) Sabemos que se $\widetilde{v}$ é uma extensão de $v$, como $K$ é o bordo de $F$, temos $\widetilde{v}$ transversal ao bordo. Assim, temos $\operatorname{Ind}_{P H}(\widetilde{v}, F)=\chi(F)$, que por sua vez, $\chi(F)=$ $\beta_{0}(F)-\beta_{1}(F)+\ldots+(-1)^{n} \beta_{n}(F)$, onde $\beta_{i}$ é o $i$-ésimo número de Betti. Como $F$ tem o tipo de homotopia de um bouquet de esferas de dimensão $n$, temos que $b_{i}=0, \forall i=$ 
$1, \ldots, n-1, b_{0}(F)=1$ e $b_{n}=\mu$. Portanto, de $(1), \operatorname{temos} \operatorname{ind}_{G S V}(v, 0)=\operatorname{Ind}_{P H}(\tilde{v}, F)=$ $1+(-1)^{n} \mu$.

(3) Suponha $v$ radial, então $\operatorname{Ind}_{S c h}(v, 0)=1$. De $(2)$, temos que

$$
\begin{aligned}
\mu & =(-1)^{n} \cdot\left(\operatorname{Ind}_{G S V}(v, 0)-1\right) \\
& =(-1)^{n} \cdot\left(\operatorname{Ind}_{G S V}(v, 0)-\operatorname{Ind}_{S c h}(v, 0)\right)
\end{aligned}
$$

Suponha agora $v$ um campo qualquer. Considere pequenas esferas $S_{\varepsilon}, S_{\varepsilon^{\prime}}, \operatorname{com} \varepsilon>\varepsilon^{\prime}>$ 0, e seja $w$ um campo no cilindro $X$ contido em $V$, limitado pelos links $K_{\varepsilon}=S_{\varepsilon} \cap V$ e $K_{\varepsilon^{\prime}}=S_{\varepsilon^{\prime}} \cap V$, tal que $w$ tem apenas um número finito $p$ de singularidades $a_{i}$ no interior de $X$ e sua restrição a $K_{\varepsilon}$ e $K_{\varepsilon^{\prime}}$ são $v$ e $v_{r a d}$, respectivamente.

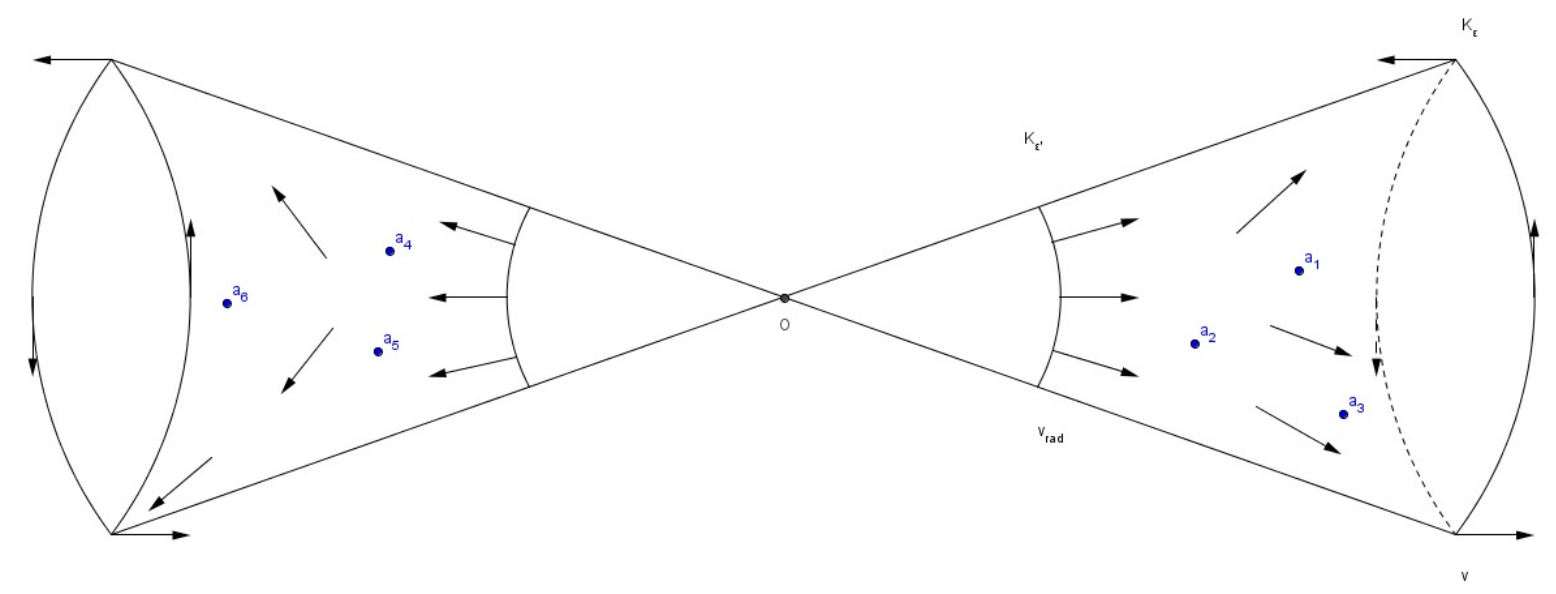

Figura 4.6: Extensão do campo radial

Assim, temos imediatamente que

$$
\operatorname{Ind}_{S c h}(v, 0)=1+d\left(v, v_{r a d}\right)=\operatorname{Ind}_{S c h}\left(v_{r a d}\right)+\sum_{i=1}^{p} \operatorname{Ind}_{P H}\left(w, a_{i}\right) .
$$

Além disso, é possível provar que

$$
\operatorname{Ind}_{G S V}(v, 0)=\operatorname{Ind}_{G S V}\left(v_{r a d}, 0\right)+\sum_{i=1}^{p} \operatorname{Ind}_{P H}\left(w, a_{i}\right) .
$$


De fato, considere $F$ como variedade diferenciável de dimensão $2 n$ e bordo $\partial F=K$. Utilizando-se do item (1) e do Teorema de Poincaré-Hopf para variedades diferenciáveis com bordo (ver [19], capítulo 6), basta encontrar um campo conveniente.

Considere sobre $V$ o campo $\widetilde{w}$ da seguinte forma: da origem à $K_{\varepsilon^{\prime}}$ colocamos $\widetilde{w}=v_{\text {rad }}$. Em $X$ colocamos $\widetilde{w}=w$. Como no bordo $\partial F$ o campo $\widetilde{w}$ coincide com $v$, podemos tomar $\widetilde{w}$ como uma extensão de $v$ à fibra $F$. Assim, temos que vale $(b)$. Portanto, de $(a)$ e $(b)$, temos

$$
\begin{aligned}
& \operatorname{Ind}_{G S V}(v, 0)-\operatorname{Ind}_{G S V}\left(v_{r a d}, 0\right)=\operatorname{Ind}_{S c h}(v, 0)-\operatorname{Ind}_{S c h}\left(v_{r a d}, 0\right) \\
& \Rightarrow \operatorname{Ind}_{G S V}(v, 0)-\operatorname{Ind}_{S c h}(v, 0)=1+(-1)^{n} \mu-1=(-1)^{n} \mu .
\end{aligned}
$$

Exemplo 4.25 : Considere $f:\left(\mathbb{C}^{2}, 0\right) \rightarrow(\mathbb{C}, 0)$ com singularidade isolada na origem e considere também o campo

$$
v(w)=\left(-\frac{\partial f}{\partial z_{2}}, \frac{\partial f}{\partial z_{1}}\right)
$$

Como vimos anteriormente, $v$ é tangente $\grave{a} V=f^{-1}(0)$ e a todas as fibras de $f$. Logo, pelo item (1) do teorema anterior,

$$
\operatorname{Ind}_{G S V}(v, 0)=\operatorname{Ind}_{P H}(\widetilde{v}, F)=0,
$$

onde $\widetilde{v}=\left.v\right|_{F}$.

Mais geralmente, seja $f:\left(\mathbb{C}^{2 n}, 0\right) \rightarrow(\mathbb{C}, 0)$ holomorfa, com singularidade isolada em 0 . Temos

$$
d f_{z}=\left(\frac{\partial f}{\partial z_{1}}, \ldots, \frac{\partial f}{\partial z_{2 n}}\right)
$$

Seja

$$
\bar{\xi}=\left(-\frac{\partial f}{\partial z_{2}}, \frac{\partial f}{\partial z_{1}},-\frac{\partial f}{\partial z_{4}}, \frac{\partial f}{\partial z_{3}},-\frac{\partial f}{\partial z_{6}}, \frac{\partial f}{\partial z_{5}}, \ldots,-\frac{\partial f}{\partial z_{2 n}}, \frac{\partial f}{\partial z_{2 n-1}}\right)
$$


Claramente temos $d f_{z}(\bar{\xi}(w))=0, \forall w \in C^{2 n}$, isto é, $\bar{\xi}$ é tangente à todas as fibras de $f$. Assim, temos $\operatorname{Ind}_{G S V}(\xi, 0)=0$, onde $\xi=\left.\bar{\xi}\right|_{V}$. Observe que também poderíamos ter tomado o campo

$$
\bar{\xi}=\left(-\frac{\partial f}{\partial z_{3}},-\frac{\partial f}{\partial z_{4}}, \frac{\partial f}{\partial z_{1}}, \frac{\partial f}{\partial z_{2}}, \ldots,-\frac{\partial f}{\partial z_{2 n-1}},-\frac{\partial f}{\partial z_{2 n}}, \frac{\partial f}{\partial z_{2 n-3}}, \frac{\partial f}{\partial z_{2 n-2}}\right)
$$

e o resultado seria o mesmo.

Teorema 4.26 : Sejam V uma variedade analítica complexa, compacta, com singularidades isoladas $x_{1}, \ldots, x_{r}$, as quais são todas germes de ICIS (nas equações reduzidas). Seja $v$ um campo contínuo em $V$, singular em $x_{1}, \ldots, x_{r}$ e também possivelmente em $y_{1}, \ldots, y_{s}$. Seja

$$
\operatorname{Ind}_{G S V}(v, V)=\sum_{i=1}^{r} \operatorname{Ind}_{G S V}\left(v, x_{i} ; V\right)+\sum_{j=1}^{s} \operatorname{Ind}_{P H}\left(v, y_{j}\right) .
$$

Então,

$$
\operatorname{Ind}_{G S V}(v, V)=\chi(V)+(-1)^{n} \cdot \sum_{i=1}^{r} \mu\left(x_{i}\right),
$$

onde $\mu\left(x_{i}\right)$ é o número de Milnor de $V$ em $x_{i}$.

Demonstração: Sabemos, pelo Teorema 4.24, que

$$
(-1)^{n} \cdot \mu\left(x_{i}\right)+\operatorname{Ind}_{S c h}\left(v, x_{i}\right)=\operatorname{Ind}_{G S V}\left(v, x_{i}\right) . \quad(* *)
$$

Substituindo $(* *)$ em $(*)$, obtemos a expressão

$$
\operatorname{Ind}_{G S V}(v, V)=\sum_{i=1}^{r} \operatorname{Ind}_{S c h}\left(v, x_{i}\right)+\sum_{j=1}^{s} \operatorname{Ind}_{P H}\left(v, y_{j}\right)+(-1)^{n} \cdot \sum_{i=1}^{r} \mu\left(x_{i}\right) .
$$

Donde, pelo Teorema 4.15, temos o desejado. 


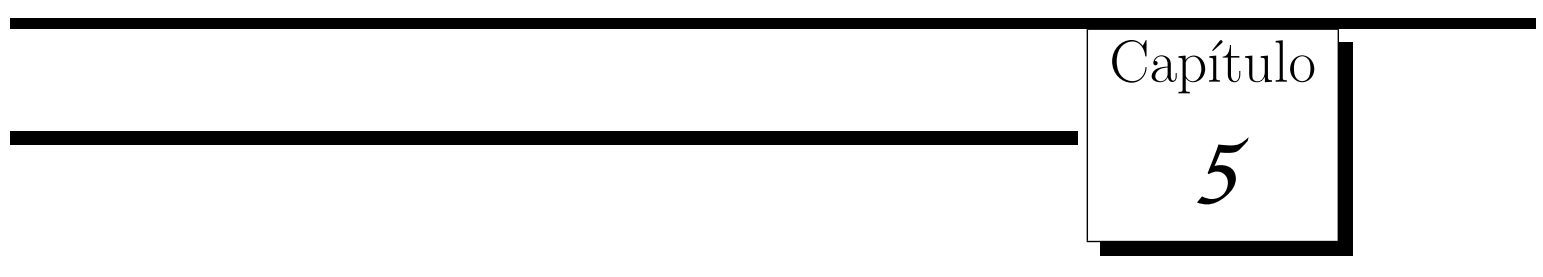

\section{Característica de Euler da fibra genérica}

\section{de ICIS simples.}

Seja $\mathcal{O}_{m, k}=\left\{f:\left(\mathbb{C}^{m}, 0\right) \rightarrow\left(\mathbb{C}^{k}, 0\right) ; f\right.$ é holomorfa $\}$. Seja $f \in \mathcal{O}_{m, k}$ tal que $V=$ $f^{-1}(0)$ define uma ICIS.

A classificação de germes em $\mathcal{O}_{m, k}$ com relação ao grupo de contato $\mathcal{K}$ definido por Mather (em [18]), é útil para estudar variedades analíticas que são ICIS.

Por esta razão, definimos aqui o grupo $\mathcal{K}$ e a $\mathcal{K}$-equivalência.

Definição 5.1 O grupo de contato é o conjunto

$$
\mathcal{K}=\left\{H:\left(\mathbb{C}^{m+k}, 0\right) \rightarrow\left(\mathbb{C}^{m+k}, 0\right) ; H\right. \text { é germe de difeomorfismo holomorfo, }
$$

com $H$ satisfazendo as seguintes condições:

i. $H(x, y)=(h(x), \theta(x, y))$,

ii. $\theta(x, 0)=0\}$

Segue das condições acima que $h:\left(\mathbb{C}^{m}, 0\right) \rightarrow\left(\mathbb{C}^{m}, 0\right)$ é também um germe de difeomorfismo.

Dizemos que $f, g \in \mathcal{O}_{m, k}$ são $\mathcal{K}$-equivalentes, e denotamos por $f \sim_{\mathcal{K}} g$, se existe $H \in \mathcal{K}$ tal que

$$
H(x, f(x))=(h(x), g(h(x))) .
$$


Observação 5.2 Se $f, g$, $h$ e $H$ são como acima, temos que $h\left(f^{-1}(0)\right)=g^{-1}(0)$.

Definição 5.3 : O espaço vetorial de todas as aplicações $f: \mathbb{C}^{m} \rightarrow \mathbb{C}^{k}$ cujas componentes são polinômios de grau menor ou igual a $r$, com $f(0)=0$, é chamado espaço de r-jatos, e a notação para tal espaço é $J^{r}(m, k)$. Os elementos de $J^{r}(m, k)$ são chamados de r-jatos.

Suponha que $f: \mathbb{C}^{m} \rightarrow \mathbb{C}^{k}$ seja uma aplicação suave e que $a \in \mathbb{C}^{m}$. Considere a série de Taylor de $f(x)-f(a)$, desta série, considere a expressão sem todos os termos maiores que $r$, para construir o $r$-jato do germe $f$ no ponto $a$, o qual será denotado por $j^{r} f(a)$.

Definição 5.4 Um germe $f \in \mathcal{O}_{m, k}$ é $\mathcal{K}$-simples se existe $r_{0} \in \mathbb{N}$ tal que se $r \geq r_{0}$, existe uma vizinhança $U$ de $j^{r} f$ em $J^{r}(m, k)$ que intersecta apenas um número finito de $\mathcal{K}$-órbitas em $J^{r}(m, k)$.

Se $f$ é $\mathcal{K}$-simples e $V=f^{-1}(0)$ é ICIS, então dizemos que $V$ é uma ICIS simples.

\section{Primeira Tabela}

O primeiro grupo de singularidades $\mathcal{K}$-simples, $f:\left(\mathbb{C}^{n}, 0\right) \rightarrow(\mathbb{C}, 0)$, é formado pelas singularidades simples de Arnol'd.

\begin{tabular}{|c|c|c|}
\hline Notação & Forma normal & Restrições \\
\hline$A_{\mu}$ & $z_{1}^{\mu+1}+Q_{n-1}$ & $\mu \geq 1$ \\
\hline$D_{\mu}$ & $z_{1}^{2} z_{2}+z_{2}^{\mu-1}+Q_{n-2}$ & $\mu \geq 4$ \\
\hline$E_{6}$ & $z_{1}^{3}+z_{2}^{4}+Q_{n-2}$ & - \\
\hline$E_{7}$ & $z_{1}^{3}+z_{1} z_{2}^{3}+Q_{n-2}$ & - \\
\hline$E_{8}$ & $z_{1}^{3}+z_{2}^{5}+Q_{n-2}$ & - \\
\hline
\end{tabular}

Tabela 5.1: Germes $\mathcal{K}$-simples $\mathbb{C}^{n} \rightarrow \mathbb{C}$. $Q_{n-i}=z_{i+1}^{2}+\cdots+z_{n}^{2}$.

Em todos os casos da Tabela 5.1 a variedade, dada pela imagem inversa do 0 pela forma normal, é uma hipersuperfície em $\mathbb{C}^{n}$. A fibra genérica

$$
F=\left\{z \in \mathbb{C}^{n} ; f(z)=\varepsilon, \varepsilon>0 \text { suficientemente pequeno }\right\}
$$


é uma variedade suave. Pelos resultados de Milnor, $\chi(F)=1+(-1)^{n-1} \cdot \mu$. O número de Milnor neste caso é dado pela codimensão do ideal gerado pelo Jacobiano da forma normal, isto é, se $f$ é a forma normal, então

$$
\mu(f)=\operatorname{dim} \frac{\mathcal{O}_{n}}{\left\langle\frac{\partial f}{\partial z_{1}}, \ldots, \frac{\partial f}{\partial z_{n}}\right\rangle} .
$$

Primeiramente, observe que fazer o cálculo acima para funções de $n$ variáveis é o mesmo que fazer para duas, visto que $Q_{n-i}$ tem apenas termos quadráticos, donde ao calcularmos o Jacobiano, temos as últimas $n-i$ variáveis no ideal $\langle J\rangle$.

Exemplo 5.5 : Seja $f\left(z_{1}, \ldots, z_{n}\right)=z_{1}^{\mu+1}+Q_{n-1}$, com $\mu \geq 1$. Temos assim que $J f=\left((\mu+1) z_{1}^{\mu}, z_{2}, z_{3}, \ldots, z_{n}\right)$. Portanto, a conta se resume a

$$
\mu(f)=\operatorname{dim} \frac{\mathcal{O}_{n}}{\left\langle\frac{\partial f}{\partial z_{1}}, \ldots, \frac{\partial f}{\partial z_{n}}\right\rangle}=\operatorname{dim} \frac{\mathcal{O}_{1}}{\left\langle z_{1}\right\rangle}=\operatorname{dim}\left\{1, z_{1}, z_{1}^{2}, \ldots, z_{1}^{\mu-1}\right\}_{\mathbb{C}}
$$

onde $\left\{1, z_{1}, z_{1}^{2}, \ldots, z_{1}^{\mu-1}\right\}_{\mathbb{C}}$ o espaço complexo gerado por estes polinômios. Assim, temos que o número de Milnor de $f$ é $\mu$, portanto, $\chi(F)=1+(-1)^{n-1} \mu$.

Exemplo 5.6 : Seja $f\left(z_{1}, \ldots, z_{n}\right)=z_{1}^{3}+z_{2}^{4}+Q_{n-2}$. Como observamos, podemos considerar $f$ como $f\left(z_{1}, z_{2}\right)=z_{1}^{3}+z_{2}^{4}$ e o número de Milnor será o mesmo. Temos $J f=\left(3 z_{1}^{2}, 4 z_{2}^{3}\right)$, o qual nos dá $\langle J f\rangle=\left\langle z_{1}^{2}, z_{2}^{3}\right\rangle$. Procedendo como no Capítulo 1 , temos

$$
\begin{aligned}
& 1 \\
& z_{1} \quad z_{2} \\
& z_{1}^{2} \quad z_{1} z_{2} \quad z_{2}^{2} \\
& z_{1}^{3} \quad z_{1}^{2} z_{2} \quad z_{1} z_{2}^{2} \quad z_{2}^{3} \\
& z_{1}^{4} \quad z_{1}^{3} z_{2} \quad z_{1}^{2} z_{2}^{2} \quad z_{1} z_{2}^{3} \quad z_{2}^{4} \\
& z_{1}^{5} \quad z_{1}^{4} z_{2} \quad z_{1}^{3} z_{2}^{2} \quad z_{1}^{2} z_{2}^{3} \quad z_{1} z_{2}^{4} \quad z_{2}^{5}
\end{aligned}
$$

donde $\operatorname{cod}_{0} f=1, \operatorname{cod}_{1} f=2, \operatorname{cod}_{2} f=2$ pois $z_{1}^{2} \in J f, \operatorname{cod}_{3} f=1$ pois $z_{2}^{3} \in J f$, $\operatorname{cod}_{4} f=0$ e portanto, o número de Milnor de $f$ é 6 . 
Exemplo 5.7 Seja $f\left(z_{1}, z_{2}\right)=z_{1}^{2} z_{2}+z_{2}^{\mu-1}$. Temos $J f=\left\langle z_{1} z_{2}, z_{1}^{2}+(\mu-1) z_{2}^{\mu-2}\right\rangle$.

Olhando para lista de monômios do exemplo anterior, temos que todos os termos mistos estão em Jf. Além disso, temos que $z_{1}^{3}=z_{1} \cdot \frac{\partial f}{\partial z_{2}}-(\mu-1) z_{1} \cdot z_{2}^{\mu-2}$, onde $(\mu-1) z_{1} \cdot z_{2}^{\mu-2}$ é um termo misto. Como $z_{1}^{2}+(\mu-1) z_{2}^{\mu-2}$ está em $J f$, temos que $z_{1}^{2}$ ou $(\mu-1) z_{2}^{\mu-2}$ não pode ser contado no cálculo de $\mu$. Portanto, retirando $z_{1}^{2}$, temos $\mu(f)=\operatorname{dim} \frac{\mathcal{O}_{n}}{\left\langle\frac{\partial f}{\partial z_{1}}, \ldots, \frac{\partial f}{\partial z_{n}}\right\rangle}=\operatorname{dim} \frac{\mathcal{O}_{2}}{\left\langle z_{1} z_{2}, z_{1}^{2}+(\mu-1) z_{2}^{\mu-2}\right\rangle}=\operatorname{dim}\left\{1, z_{1}, z_{2}, z_{2}^{2}, \ldots, z_{2}^{\mu-2}\right\}_{\mathbb{C}}=\mu$

Utilizando o programa Maple, calculamos os demais números de Milnor da Tabela 5.1. obtendo assim uma nova tabela

\begin{tabular}{|c|c|c|c|c|}
\hline Notação & Forma normal & Restrições & $\mu(f)$ & $\chi(F)$ \\
\hline$A_{\mu}$ & $z_{1}^{\mu+1}+Q_{n-1}$ & $\mu \geq 1$ & $\mu$ & $1+(-1)^{n-1} \mu$ \\
\hline$D_{\mu}$ & $z_{1}^{2} z_{2}+z_{2}^{\mu-1}+Q_{n-2}$ & $\mu \geq 4$ & $\mu$ & $1+(-1)^{n-1} \mu$ \\
\hline$E_{6}$ & $z_{1}^{3}+z_{2}^{4}+Q_{n-2}$ & - & 6 & $1+(-1)^{n-1} 6$ \\
\hline$E_{7}$ & $z_{1}^{3}+z_{1} z_{2}^{3}+Q_{n-2}$ & - & 7 & $1+(-1)^{n-1} 7$ \\
\hline$E_{8}$ & $z_{1}^{3}+z_{2}^{5}+Q_{n-2}$ & - & 8 & $1+(-1)^{n-1} 8$ \\
\hline
\end{tabular}

\section{Segunda Tabela}

A Tabela 5.3 contém as interseções completas 0-dimensionais que são $\mathcal{K}$-simples.

\begin{tabular}{|c|c|c|}
\hline Notação & Forma normal & Restrições \\
\hline$C_{k, l}^{ \pm}$ & $\left(z_{1} z_{2}, z_{1}^{k}+z_{2}^{l}\right)$ & $l \geq k \geq 2$ \\
\hline$\widetilde{C}_{2 k}$ & $\left(z_{1}^{2}+z_{2}^{2}, z_{2}^{k}\right)$ & $k \geq 3$ \\
\hline$F_{2 m+1}$ & $\left(z_{1}^{2}+z_{2}^{3}, z_{2}^{m}\right)$ & $m \geq 3$ \\
\hline$F_{2 m+4}$ & $\left(z_{1}^{2}+z_{2}^{3}, z_{1} z_{2}^{m}\right)$ & $m \geq 2$ \\
\hline$G_{10}^{*}$ & $\left(z_{1}^{2}, z_{2}^{4}\right)$ & - \\
\hline$H_{m+5}^{ \pm}$ & $\left(z_{1}^{2}+z_{2}^{m}, z_{1} z_{2}^{2}\right)$ & $m \geq 4$ \\
\hline
\end{tabular}

Tabela 5.2: Germes $\mathcal{K}$-simples $\mathbb{C}^{2} \rightarrow \mathbb{C}^{2}$.

Para ICIS 0-dimensionais, definidas por $f: \mathbb{C}^{n} \rightarrow \mathbb{C}^{n}$, temos $f^{-1}(0)=0$. A fibra $F=f^{-1}(\varepsilon), \operatorname{com} \varepsilon \neq 0$, é um número finito de pontos. Para $\varepsilon$ e $\varepsilon^{\prime}$ genéricos, temos que 
$\sharp f^{-1}(\varepsilon)=\sharp f^{-1}\left(\varepsilon^{\prime}\right)$ e é chamado a multiplicidade de $V$ (ou de $f$ ). Esta multiplicidade, que denotaremos por $m_{0}(f)$, é dada por

$$
m_{0}(f)=\operatorname{dim}_{\mathbb{C}} \frac{\mathcal{O}_{n}}{\left\langle f_{1}, \ldots, f_{n}\right\rangle}, \quad \text { onde } f=\left(f_{1}, \ldots, f_{n}\right)
$$

Neste caso, como a dimensão da variedade é 0 , temos o seguinte resultado

Proposição 5.8 Se $V=f^{-1}(0)$ é uma ICIS 0-dimensional, então $\chi(F)=m_{0}(f)=$ $1+\mu(f)$.

Deste modo, pela proposição acima, para calcular $\chi(F)$, basta calcular $m_{0}(f)$. Vamos calcular esta multiplicidade para $C_{k, l}^{ \pm}$e $F_{2 m+1}$, as demais contas foram feitas utilizando o Maple.

Singularidades simples de ICIS tem formas normais especiais, representadas por funções polinomiais quase homogêneas. Assim, para fazer o calculo do número de Milnor nestes casos, podemos utilizar a fórmula de Milnor Orlik.

Definição 5.9 Uma aplicação $f=\left(f_{1}, \ldots, f_{k}\right) \in \mathcal{O}_{n, k}$ é quase-homogênea de tipo $\left(w_{1}, w_{2}, \ldots, w_{n} ; d_{1}, d_{2}, \ldots, d_{k}\right) s e$

$$
f_{i}\left(\lambda^{w_{1}} z_{1}, \lambda^{w_{2}} z_{2}, \ldots, \lambda^{w_{n}} z_{n}\right)=\lambda^{d_{i}} f_{i}\left(z_{1}, z_{2}, \ldots, z_{n}\right), \quad \forall i=1, \ldots, k
$$

Teorema 5.10: (Milnor-Orlik) Seja $f=\left(f_{1}, \ldots, f_{n}\right):\left(\mathbb{C}^{n}, 0\right) \rightarrow\left(\mathbb{C}^{n}, 0\right)$ quasehomogênea de tipo $\left(w_{1}, w_{2}, \ldots, w_{n} ; d_{1}, d_{2}, \ldots, d_{n}\right)$. Suponha $m_{0}(f)$ finito. Então

$$
m_{0}(f)=\frac{d_{1} \cdot d_{2} \cdot \ldots \cdot d_{n}}{w_{1} \cdot w_{2} \cdot \ldots \cdot w_{n}}
$$

Exemplo 5.11 : Consideremos a primeira função da segunda tabela $f\left(z_{1}, z_{2}\right)=\left(z_{1} z_{2}, z_{1}^{k}+\right.$ $\left.z_{2}^{l}\right)$. Note que todos os termos mistos $z_{1}^{i} z_{2}^{j}$, com $i, j>0$, pertencem $\grave{a}\left\langle z_{1} z_{2}, z_{1}^{k}+z_{2}^{l}\right\rangle$. Note que $z_{1}^{k+1}=z_{1}\left(z_{1}^{k}+z_{2}^{l}\right)-z_{1} z_{2}^{l}$, onde $z_{1} z_{2}^{l} \in\left\langle z_{1} z_{2}, z_{1}^{k}+z_{2}^{l}\right\rangle$, portanto $z_{1}^{k+1} \in$ $\frac{\mathcal{O}_{2}}{\left\langle z_{1} z_{2}, z_{1}^{k}+z_{2}^{l}\right\rangle}$. O mesmo vale para $z_{2}^{k+1}$. Novamente, como $z_{1}^{k}+z_{2}^{l}$ está em $\left\langle z_{1} z_{2}, z_{1}^{k}+z_{2}^{l}\right\rangle$, 
temos que retirar um no cálculo de $m_{0}$. Deste modo, retirando $z_{2}^{l}$, temos

$$
\frac{\mathcal{O}_{2}}{\left\langle z_{1} z_{2}, z_{1}^{k}+z_{2}^{l}\right\rangle}=\left\{1, z_{1}, z_{1}^{2}, \ldots, z_{1}^{k}, z_{2}, z_{2}^{2}, \ldots, z_{2}^{l-1}\right\}_{\mathbb{C}}
$$

Portanto, $\chi(F)=k+l$ e $\mu(f)=k+l-1$.

Exemplo 5.12 Seja $f\left(z_{1}, z_{2}\right)=\left(z_{1}^{2}+z_{2}^{2}, z_{2}^{k}\right)$. Note que $f$ é quase-homogênea (na verdade homogênea) de tipo $(1,1 ; 2, k)$. Assim, pelo Teorema 5.10, temos que $m_{0}(f)=$ 2k. Portanto, $\chi(F)=2 k$ e $\mu(f)=2 k-1$.

Exemplo 5.13 Seja $f\left(z_{1}, z_{2}\right)=\left(z_{1}^{2}+z_{2}^{3}, z_{2}^{m}\right)$, com $m \geq 3$. Queremos computar então

$$
\frac{\mathcal{O}_{2}}{\left\langle z_{1}^{2}+z_{2}^{3}, z_{2}^{m}\right\rangle} \text {. }
$$

Olhando para a mesma lista de monômios do Exemplo 5.6, temos que $\operatorname{cod}_{0} f=1$, $\operatorname{cod}_{1} f=2$. Note que $z_{1}^{2}=f_{1}\left(z_{1}, z_{2}\right)-z_{2}^{3}$, onde $z_{2}^{3} \in \mathfrak{M}_{2}^{3}$. Procedendo deste modo, vamos encontrar exatamente 2 elementos em cada linha, sendo eles $z_{1} z_{2}^{j}$ e $z_{2}^{j+1}$, com $1 \leq j \leq m-1$. Por fim, na $m$-ésima linha, temos $z_{2}^{m} \in\langle J\rangle$, donde $\operatorname{cod}_{m+1} f=1$. Assim, $m_{0}=1+\underbrace{2+2+\ldots+2}_{m-1 \text { vezes }}+1=2 m$ e $\mu(f)=2 m-1$.

Exemplo 5.14 Seja $f\left(z_{1}, z_{2}\right)=\left(z_{1}^{2}+z_{2}^{3}, z_{1} z_{2}^{m}\right)$. Temos que $f$ é quase homogênea de tipo $(3,2 ; 6,3+2 m)$. Assim,

$$
m_{0}=\frac{6(3+2 m)}{6}=3+2 m
$$

Portanto $\chi(F)=2 m+3$ e $\mu(f)=2 m+2$.

Utilizando novamente o Maple, obtemos a seguinte tabela

\section{Terceira Tabela}

Para $f=\left(f_{1}, f_{2}\right)$ na Tabela 5.4 , temos que $V=f^{-1}(0)$ é uma curva complexa em $\mathbb{C}^{3}$, que é uma ICIS. Sejam $\Sigma(f)$ o conjunto singular de $f$ e $\Delta(f)=f(\Sigma(f))$ a imagem 


\begin{tabular}{|c|c|c|c|c|}
\hline Notação & Forma normal & Restrições & $\chi(F)$ & $\mu(f)$ \\
\hline$C_{k, l}^{ \pm}$ & $\left(z_{1} z_{2}, z_{1}^{k}+z_{2}^{l}\right)$ & $l \geq k \geq 2$ & $k+l$ & $k+l-1$ \\
\hline$\widetilde{C}_{2 k}$ & $\left(z_{1}^{2}+z_{2}^{2}, z_{2}^{k}\right)$ & $k \geq 3$ & $2 k$ & $2 k-1$ \\
\hline$F_{2 m+1}$ & $\left(z_{1}^{2}+z_{2}^{3}, z_{2}^{m}\right)$ & $m \geq 3$ & $2 m$ & $2 m-1$ \\
\hline$F_{2 m+4}$ & $\left(z_{1}^{2}+z_{2}^{3}, z_{1} z_{2}^{m}\right)$ & $m \geq 2$ & $2 m+3$ & $2 m+2$ \\
\hline$G_{10}^{*}$ & $\left(z_{1}^{2}, z_{2}^{4}\right)$ & - & 8 & 7 \\
\hline$H_{m+5}^{ \pm}$ & $\left(z_{1}^{2}+z_{2}^{m}, z_{1} z_{2}^{2}\right)$ & $m \geq 4$ & $m+2$ & $m+1$ \\
\hline
\end{tabular}

Tabela 5.3: Germes $\mathcal{K}$-simples $\mathbb{C}^{2} \rightarrow \mathbb{C}^{2}$.

\begin{tabular}{|c|c|c|}
\hline Notação & Forma normal & Restrições \\
\hline$S_{\mu}$ & $\left(z_{1}^{2}+z_{2}^{2}+z_{3}^{\mu-3}, z_{2} z_{3}\right)$ & $\mu \geq 5$ \\
\hline$T_{7}$ & $\left(z_{1}^{2}+z_{2}^{3}+z_{3}^{3}, z_{2} z_{3}\right)$ & - \\
\hline$T_{7}$ & $\left(z_{1}^{2}+z_{2}^{2}, z_{2}^{2}+z_{3}^{2}\right)$ & - \\
\hline$T_{8}$ & $\left(z_{1}^{2}+z_{2}^{3}+z_{3}^{4}, z_{2} z_{3}\right)$ & - \\
\hline$T_{9}$ & $\left(z_{1}^{2}+z_{2}^{3}+z_{3}^{5}, z_{2} z_{3}\right)$ & - \\
\hline$U_{7}$ & $\left(z_{1}^{2}+z_{2} z_{3}, z_{1} z_{2}+z_{3}^{3}\right)$ & - \\
\hline$U_{8}$ & $\left(z_{1}^{2}+z_{2} z_{3}+z_{3}^{3}, z_{1} z_{2}\right)$ & - \\
\hline$U_{9}$ & $\left(z_{1}^{2}+z_{2} z_{3}, z_{1} z_{2}+z_{3}^{4}\right)$ & - \\
\hline$W_{8}$ & $\left(z_{1}^{2}+z_{2}^{3}, z_{2}^{2}+z_{1} z_{3}\right)$ & - \\
\hline$W_{9}$ & $\left(z_{1}^{2}+z_{2} z_{3}^{2}, z_{2}^{2}+z_{1} z_{3}\right)$ & - \\
\hline$Z_{9}$ & $\left(z_{1}^{2}+z_{3}^{3}, z_{2}^{2}+z_{3}^{3}\right)$ & - \\
\hline$Z_{10}$ & $\left(z_{1}^{2}+z_{2} z_{3}^{2}, z_{2}^{2}+z_{3}^{3}\right)$ & - \\
\hline
\end{tabular}

Tabela 5.4: Germes $\mathcal{K}$-simples $\mathbb{C}^{3} \rightarrow \mathbb{C}^{2}$.

deste conjunto singular pela $f$. Se $\varepsilon \notin \Delta(f)$, então $F=f^{-1}(\varepsilon)$ é a fibra genérica de $f$. Assim, novamente vale o Teorema 4.24, isto é,

$$
\chi(F)=1+(-1)^{n} \mu(f)=1-\mu(f) .
$$

O problema é determinar o número de Milnor de $f$. Para isto, vamos usar a fórmula indutiva de Lê-Greuel (ver [17]).

Teorema 5.15: (Lê-Greuel) Seja $\pi: \mathbb{C}^{3} \rightarrow \mathbb{C}$ dada por $\pi\left(z_{1}, z_{2}, z_{3}\right)=a z_{1}+b z_{2}+c z_{3}$, uma projeção linear genérica, com $a, b, c$ genéricos. Então

$$
\mu(f)+\mu(f, \pi)=\operatorname{dim}_{\mathbb{C}} \frac{\mathcal{O}_{3}}{\langle f, J(f, \pi)\rangle},
$$


onde $(f, \pi): \mathbb{C}^{3} \rightarrow \mathbb{C}^{3}$ é dada por $(f, \pi)\left(z_{1}, z_{2}, z_{3}\right)=\left(f_{1}\left(z_{1}, z_{2}, z_{3}\right), f_{2}\left(z_{1}, z_{2}, z_{3}\right), \pi\left(z_{1}, z_{2}, z_{3}\right)\right)$.

Note que $\mu(f, \pi)=m_{0}(f, \pi)-1$, pois $(f, \pi)^{-1}(0)$ é uma ICIS 0-dimensional.

Exemplo 5.16 Seja $f\left(z_{1}, z_{2}, z_{3}\right)=\left(z_{1}^{2}+z_{2}^{2}+z_{3}^{\mu-3}, z_{2} \cdot z_{3}\right)$, com $\mu \geq 5$. É possível mostrar que o resultado não depende dos termos a, $b$, c escolhidos para $\pi$, desde que os mesmos sejam escolhidos de forma correta (pelo Teorema de Sard, o conjunto ruim para escolha destas constantes tem medida nula). Neste exemplo, podemos tomar $b=c=0$ e $a=1$, o que nos dá $\pi\left(z_{1}, z_{2}, z_{3}\right)=z_{1}$. Assim,

$$
\frac{\mathcal{O}_{3}}{\left\langle z_{1}^{2}+z_{2}^{2}+z_{3}^{\mu-3}, z_{1} z_{2}, z_{1}\right\rangle} \simeq \frac{\mathcal{O}_{2}}{\left\langle z_{2}^{2}+z_{3}^{\mu-3}, z_{2} z_{3}\right\rangle}
$$

Pelo Exemplo5.11 temos que $m_{0}(f, \pi)=4$ se $\mu=5$ e $m_{0}(f, \pi)=\mu-3+2=\mu-1$, se $\mu>5$. Agora temos que calcular $\frac{\mathcal{O}_{3}}{\langle f, J(f, \pi)\rangle}$, onde

$$
J(f, \pi)=\operatorname{det}\left(\left[\begin{array}{ccc}
2 z_{1} & 2 z_{2} & (\mu-3) z_{3}^{\mu-4} \\
0 & z_{3} & z_{2} \\
1 & 0 & 0
\end{array}\right]\right)=2 z_{2}^{2}-(\mu-3) z_{3}^{\mu-3}
$$

Aplicando o Teorema de Milnor-Orlik 5.10 para o germe

$$
\left(z_{1}^{2}+z_{2}^{2}+z_{3}^{\mu-3}, z_{2} z_{3}, 2 z_{2}^{2}-(\mu-3) z_{3}^{\mu-3}\right)
$$

que é quase-homogêneo de tipo $(\mu-3, \mu-3,2 ; 2(\mu-3), \mu-1,2(\mu-3))$, temos que $\operatorname{dim}_{\mathbb{C}} \frac{\mathcal{O}_{3}}{\left\langle z_{1}^{2}+z_{2}^{2}+z_{3}^{\mu-3}, z_{2} z_{3}, 2 z_{2}^{2}-(\mu-3) z_{3}^{\mu-3}\right\rangle}=\frac{2 \cdot(\mu-3) \cdot(\mu-1) \cdot 2 \cdot(\mu-3)}{(\mu-3) \cdot(\mu-3) \cdot 2}=2(\mu-1)$

Portanto, se $\mu=5$, temos que $\mu(f)+4=8, \operatorname{logo} \mu(f)=4$. Se $\mu>5$, então temos $\mu(f)+\mu-2=2 \mu-2, \log \circ \mu(f)=\mu$.

Exemplo 5.17 Seja $f\left(z_{1}, z_{2}, z_{3}\right)=\left(z_{1}^{2}+z_{2}^{3}+z_{3}^{4}, z_{2} z_{3}\right)$. Vamos escolher $\pi\left(z_{1}, z_{2}, z_{3}\right)=$ 
$z_{1}-z_{2}-z_{3}$. Deste modo, aplicando o Teorema 5.10, temos

$$
\operatorname{dim}_{\mathbb{C}} \frac{\mathcal{O}_{3}}{\left\langle z_{1}^{2}+z_{2}^{3}+z_{3}^{4}, z_{2} z_{3}, z_{1}-z_{2}-z_{3}\right\rangle} \simeq \operatorname{dim}_{\mathbb{C}} \frac{\mathcal{O}_{2}}{\left\langle z_{2}^{2}+z_{3}^{2}+z_{2}^{3}+z_{3}^{4}, z_{2} z_{3}\right\rangle}=\frac{12 \cdot 7}{4 \cdot 3}=4
$$

Calculando $J(f, \pi)$, temos

$$
J(f, \pi)=\operatorname{det}\left(\left[\begin{array}{ccc}
2 z_{1} & 3 z_{2}^{2} & 4 z_{3}^{3} \\
0 & z_{3} & z_{2} \\
1 & -1 & -1
\end{array}\right]\right)=2 z_{1}\left(z_{2}-z_{3}\right)+3 z_{2}^{3}-4 z_{3}^{4} .
$$

Seja $J=(f, J(f, \pi))=\left(z_{1}^{2}+z_{2}^{3}+z_{3}^{4}, z_{2} z_{3}, 2 z_{1}\left(z_{2}-z_{3}\right)+3 z_{2}^{3}-4 z_{3}^{4}\right)$. Assim,

$$
\begin{aligned}
\operatorname{dim}_{\mathbb{C}} \frac{\mathcal{O}_{3}}{J} & =\operatorname{dim}_{\mathbb{C}} \frac{\mathcal{O}_{3}}{\left\langle z_{1}^{2}+z_{2}^{3}+z_{3}^{4}, z_{2}, 2 z_{1}\left(z_{2}-z_{3}\right)+3 z_{2}^{3}-4 z_{3}^{4}\right\rangle}+\operatorname{dim}_{\mathbb{C}} \frac{\mathcal{O}_{3}}{\left\langle z_{1}^{2}+z_{2}^{3}+z_{3}^{4}, z_{3}, 2 z_{1}\left(z_{2}-z_{3}\right)+3 z_{2}^{3}-4 z_{3}^{4}\right\rangle} \\
& =\operatorname{dim}_{\mathbb{C}} \frac{\mathcal{O}_{3}}{\left\langle z_{1}^{2}+z_{3}^{4},-2 z_{1} z_{3}-4 z_{3}^{4}\right\rangle}+\operatorname{dim}_{\mathbb{C}} \frac{\mathcal{O}_{3}}{\left\langle z_{1}^{2}+z_{2}^{3}, 2 z_{1} z_{2}+3 z_{2}^{3}\right\rangle} \\
& =6+5=11
\end{aligned}
$$

Então $\mu(f)=11-4=7$. 
80CAPÍTULO 5. CARACTERÍSTICA DE EULER DA FIBRA GENÉRICA DE ICIS SIMPLES. 


\section{Referências Bibliográficas}

[1] Bonatti, Ch.; Gómez-Mont, X. The Index of a Holomorphic Vector Field on a Singular Variety, 222, Astérisque, 9 - 35, 1994.

[2] Brasselet, J. P. Classes Características de Variedades Singulares, Notas didáticas, 56, ICMC-USP, 2002.

[3] Brasselet, J. P.; Seade, J.; Suwa, T. Vector Fields on Singular Varieties, Springer, 2009.

[4] Dalbelo, T. M. O Índice de Poincaré-Hopf e Generalizações no caso Singular. 2011. 143f. Dissertação (Mestrado em Matemática) - Instituto de Ciências Matemáticas e Computação, Universidade de São Paulo, São Carlos, 2011.

[5] Gibson, C. G.; Wirthmuller, K.; Plessis, A.du; Looijenga, E.J. Topological stability of smooth mappins, LNM 552, Springer-Verlag. 1976.

[6] Gibson, C. G. Singular Points of Smooth Mappings, Research Notes in Mathematics, 25, 1979.

[7] Goryunov, V. V. Singularities of Projections of Full Intersections, 27, J. Sov. Math , 2785 - 2811, 1984.

[8] Gunning, R.; Rossi, H. Analytic Functions of Several Complex Variables, Prentice-Hall, INC. Englewood Cliffs, N.J., 1965.

[9] Hatcher, A. Algebraic Topology, Cambridge University Press, 2002.

[10] James, I.M. The Topology of Stiefel Manifolds, Cambridge University Press, 1976. 
[11] Kendig, K. Elementary Algebraic Geometry, Graduate Texts in Mathematics, 44, Springer, 1938.

[12] Lang, S. Algebra, Graduate Texts in Mathematics, 211, Springer, 1993.

[13] Lang, S. Introduction to Algebraic Geometry, Interscience Publishers, 1958.

[14] Lima, E. L. A característica de Euler-Poincaré, Rio de Janeiro, Coleção Matemática Universitária, v.1, 47-62. 1985.

[15] Lima, E. L. Curso de Análise, vol. 2, Coleção Projeto Euclides, IMPA, 1981.

[16] Lima, E. L. Variedades Diferenciáveis, Publicações Matemáticas, IMPA, 2007.

[17] Looijenga, E. J. N. Isolated Singular Points of Complete Intersections, London Mathematical Society, Lectures Notes Series 77, 1984.

[18] Mather, J. N. Stability of $C^{\infty}$ mappings, III: Finitely determined map-germs, Publications mathématiques de l'I.H.É.S., book 35, 127 - 156, 1968.

[19] Milnor, J. W. Topology from the Differentiable Viewpoint, University Press of Virginia, 1965.

[20] Milnor, J. W. Singular Points of Complex Hypersurfaces, Princeton University Press and University of Tokio Press, 1968.

[21] Nestruev, J. Smooth Manifolds and Observables, Graduate Texts in Mathematics, 220, Berlin, Springer, 2002.

[22] Steenrod, N. The Topology of Fibre Bundles, Princenton University Press, 1951.

[23] Whitney, H. Elementary Structure of Real Varieties, Ann. of Math, 66, 545-556, 1957. 